\title{
A quantum kinematics for asymptotically flat spacetimes
}

\author{
Miguel Campiglia, ${ }^{1,2}$, and Madhavan Varadarajan,1, \\ ${ }^{1}$ Raman Research Institute, Bangalore 560080, India \\ ${ }^{2}$ Instituto de Física, Facultad de Ciencias, Montevideo 11400, Uruguay
}

We construct a quantum kinematics for asymptotically flat spacetimes based on the Koslowski-Sahlmann (KS) representation. The KS representation is a generalization of the representation underlying Loop Quantum Gravity (LQG) which supports, in addition to the usual LQG operators, the action of 'background exponential operators' which are connection dependent operators labelled by 'background' $s u(2)$ electric fields. KS states have, in addition to the LQG state label corresponding to 1 dimensional excitations of the triad, a label corresponding to a 'background' electric field which describes 3 dimensional excitations of the triad. Asymptotic behaviour in quantum theory is controlled through asymptotic conditions on the background electric fields which label the states and the background electric fields which label the operators. Asymptotic conditions on the triad are imposed as conditions on the background electric field state label while confining the LQG spin net graph labels to compact sets. We show that KS states can be realised as wave functions on a quantum configuration space of generalized connections and that the asymptotic behaviour of each such generalized connection is determined by that of the background electric fields which label the background exponential operators. Similar to the spatially compact case, the Gauss Law and diffeomorphism constraints are then imposed through Group Averaging techniques to obtain a large sector of gauge invariant states. It is shown that this sector supports a unitary action of the group of asymptotic rotations and translations and that, as anticipated by Friedman and Sorkin, for appropriate spatial topology, this sector contains states which display fermionic behaviour under $2 \pi$ rotations.

\section{INTRODUCTION}

Isolated gravitating systems are modelled by asymptotically flat spacetimes. Key physical results such as the positivity of energy and the definition of total angular momentum depend on the delicate asymptotic behaviour of the gravitational field. Therefore, an understanding of quantum gravitational effects in the context of asymptotic flatness requires that these asymptotic conditions be suitably incorporated in quantum theory. The particular quantum gravity approach we are interested in is canonical Loop Quantum Gravity (LQG). LQG is an attempt at canonical quantization of a classical formulation of gravity in terms of $S U(2)$ connections and conjugate electric (triad) fields on a Cauchy slice $\Sigma$. The basic connection dependent operators are holonomies associated with graphs and the basic electric field dependent operators are fluxes through surfaces, the graphs and surfaces being embedded in the Cauchy slice. The LQG representation can be viewed as a connection representation [1].

*Electronic address: campi@fisica.edu.uy

$\dagger$ Electronic address: madhavan@rri.res.in 
A spin network basis of quantum states can be built out of holonomies of (generalized) connections [2, 3]. Consequently each such state 'lives' on a graph. Holonomy operators act by multiplication and electric fluxes through surfaces act, roughly speaking, by differentiation whenever the surface and the spin net graph intersect [2, 4] so that the triad field is excited only along the support of the graph and vanishes outside this support. As a result, the microsocopic quantum spatial geometry can be thought of as vanishing everywhere except along the spin net graph.

Most work in LQG has been in the context of compact Cauchy slices wherein the spin net graphs are chosen to have a finite number of compactly supported edges. In the asymptotically flat case, while the connection vanishes at spatial infinity, the triad approaches a fixed flat triad [5]. Since the spatial geometry is excited only along the support of the graph underlying a spin net state, and since, in the asymptotically flat case, the boundary conditions indicate that the spatial geometry is excited in non-compact regions, it follows that the graphs underlying spin net states appropriate to asymptotic flatness must be supported in non-compact regions. Thus these graphs must have infinitely many (or infinitely long) edges. Since it seems natural that, as in the spatially compact case, such spin net states should be mapped to each other by holonomy operators, it seems necessary that the compact graph holonomy operators also admit a generalization to ones supported on non-compact graphs.

In this work, instead of attempting a generalization based on non-compact versions of the standard LQG states (namely, spin nets supported on graphs with non-compact support) and the standard LQG operators (namely holonomies on non-compact graphs), we adapt a generalization of LQG itself, on compact spaces, to the asymptotically flat case. This generalization was introduced by Koslowski and further developed by Sahlmann in their seminal contributions [6 8] and we refer to it as the Koslowski-Sahlmann (KS) representation. The KS representation involves, in addition to the standard probes of LQG, an extra set of probes consisting of electric fields. Each such electric field $\bar{E}$ (called a 'background electric field' to distinguish it from the dynamical electric field which is a phase space variable) probes the behaviour of the connection $A_{a}^{i}$ through the 'background exponential function' $\beta_{\bar{E}}(A)=\exp \left(i \int_{\Sigma} \bar{E}_{i}^{a} A_{a}^{i}\right)$. The KS representation supports the action of the corresponding 'background exponential operators' [12] and the representation itself can be seen as a representation of a 'holonomy-background exponential-flux' algebra [13].

In our generalization of the KS representation to the asymptotically flat case, we demand that these extra probes (namely, the background electric fields) satisfy appropriate asymptotic conditions. These conditions on the background electric fields ensure that the background exponential functions are sensitive to the detailed asymptotic behavior of the classical connections and we are able to continue to use compactly supported graphs to define holonomies. It then turns out that in the quantum theory these asymptotic conditions on the background electric fields control the behaviour of the generalised connections which constitute the quantum configuration space. This is similar to what happens in standard flat spacetime quantum field theory where the behaviour of the elements of the quantum configuration space is determined by that of the 'dual probes'; for example, in the standard Fock representation of the scalar field, the precise distributional nature of the quantum scalar fields is determined by the rapid fall off property of the dual smearing functions [25]. As we shall show, our particular choice of asymptotic behaviour for the background electric fields which label the background exponential operators implies that elements of the quantum configuration space satisfy a quantum analog of the classical asymptotic behaviour. It 
is in this way that the classical asymptotic conditions on the connection leave their imprint in quantum theory.

Background electric fields also appear as labels of KS states in addition to the standard LQG spin net labels. The action of the electric flux operators on such states acquires, relative to standard LQG, an extra contribution corresponding to the flux of the background electric field which labels the state [6, 7]. Similar contributions ensue for the Area and Volume operators [7]. It then follows that the classical asymptotic conditions on the electric field can be incorporated in quantum theory by directly imposing them as conditions on the background electric field label of the state. These conditions are that the electric field asymptotes to the sum of a fixed flat triad field and a subleading piece [5, 18, 19. ${ }^{1}$ The background electric fields which label the background exponential operators obey the same conditions as the background electric fields which label the states except that the 'zeroth order' flat triad piece of the latter is absent in the former. As we shall see, ${ }^{2}$ it is this difference in the two sets of conditions (namely on state and operator labels) which allows their consistent imposition.

The resulting quantum kinematics supports a unitary representation of $S U(2)$ rotations and diffeomorphisms subject to appropriate asymptotic behavior. We shall be interested in $S U(2)$ rotations and diffeomorphisms which are connected to identity. The diffeomorphisms asymptote to a combination of asymptotic rotations, translations and odd supertranslations [19]. The $S U(2)$ rotations are trivial at infinity except when they act in combination with diffeomorphisms with non-trivial asymptotic rotational action so that the triad at infinity is kept fixed under this combined action. The unitary transformations corresponding to any combination of $S U(2)$ rotations which are trivial at infinity and diffeomorphisms with trivial asymptotic rotational and translational parts are interpreted as the quantum analog of the finite gauge transformations generated by the $S U(2)$ Gauss Law and spatial diffeomorphism constraints [19]. The unitary transformations corresponding to any combination of diffeomorphisms with non-trivial asymptotic rotational and translational parts together with appropriate $S U(2)$ rotations (so that the combination is an asymptotic symmetry of the fixed flat triad at infinity) are interpreted as the quantum analog of the finite symmetry transformations generated by the the total angular and linear momenta of the classical spacetime [19]. We view the ability of the quantum kinematics to support these unitary actions as an a posteriori justification for our treatment of asymptotic behaviour in quantum theory. As in the spatially compact case [7, 9], gauge invariant states can then be constructed by group averaging methods. We construct large 'superselected' sectors [9] of such group averaged states and show that these sectors support a unitary representation of the finite dimensional group of translations and rotations at infinity. As in LQG, and as in the KS representation for the compact case, the Hamiltonian constraint remains a key open issue. This, in turn, precludes a quantum analysis of the total energy and of the generator of boosts.

While this work seeks to address the construction of a quantum kinematics appropriate to asymptotic flatness, it is based on the KS representation rather than the standard LQG

\footnotetext{
${ }^{1}$ We use 'parity' conditions which are closely related to those of Reference [19] (see section II B for what we mean by the phrase 'closely related' and section IX for related issues). Parity conditions on metric variables were introduced in [16] and adapted to the self dual Ashtekar variables in [18]. The treatment of [16] was improved in [17] and the improved treatment adapted to the Ashtekar-Barbero variables in [19].

${ }^{2}$ See Footnote 9, section III A
} 
one. Since we view the KS representation as an effective description of fundamental LQG states which describe an effectively smooth spatial geometry, we would like to base our constructions on purely LQG type probes, which, as mentioned above, must now include non-compact graphs. There have been a few extremely interesting and important attempts at initiating a study of asymptotically flat LQG [10, 11] to accommodate non-compact graphs. Since these works do not address the full set of asymptotic conditions adequately, we hope that the results of this work will provide a useful supplement to that of References [10, 11] in the putative construction of an LQG quantum kinematics for asymptotically flat spacetimes.

Our work as presented here crucially relies on our recent work [9, 12, 13]. Specifically, the considerations of sections IV- VI of this paper rest on the analogous results and constructions for the compact case as detailed in Reference [13] and those of section VIII on the group averaging analysis of [9]. ${ }^{3}$ The layout of the paper is as follows.

Section II is devoted to a description of classical structures of relevance to the construction of the KS representation in the asymptotically flat case. Standard definitions of asymptotic flatness (see for example [5, 16, 17]) implicitly use the $C^{\infty}$ category. In anticipation of the formulation of the KS representation based on semianalytic, and hence, finitely differentiable fields, we tailor the definition of asymptotically flat 3-manifolds to the semianalytic category and discuss the differential properties of fields supported on such manifolds. This is done in section IIA. This section also establishes our notation for asymptotic fall-off properties of such fields. Next, in section II B we discuss the asymptotic conditions on the phase space variables, namely the $S U(2)$ connection and its conjugate electric field, ${ }^{4}$ and display the elementary phase space functions of interest, namely, the holonomies, background exponentials and electric fluxes. The 'probes' associated with these functions are, respectively, edges $e$, background electric fields $\bar{E}$ and $S U(2)$ Lie algebra valued scalars $f$ supported on surfaces $S$. Section II C discusses the precise defining properties of probes for the connection (namely $e, \bar{E}$ ) and reviews the structures associated with these probes, namely the groupoid of compactly supported paths and the Abelian group (under addition) of these electric fields subject to appropriate asymptotic conditions. In section II D we detail our choice of electric field probes $(f, S)$. In section IIE we discuss the classical Poisson brackets between the holonomies, background exponentials and electric fluxes.

In section III we define the KS representation (which as we show in this and subsequent sections is) appropriate to the asymptotically flat case in terms of the action of the holonomy, background exponential and electric flux operators on KS spin nets. Each KS spin net is labelled by a standard LQG spin net label whose underlying graph is compactly supported and a background electric field label which is subject to the same asymptotic conditions as are satisfied by the asymptotically flat electric fields which serve as classical phase space variables.

Sections [V] to $\mathrm{VI}$ are devoted to the study of the quantum configuration space and to a characterization of the asymptotic behavior of the generalised connections which constitute this space. We use $C^{*}$ algebraic methods to show that the KS representation is unitarily equivalent to one based on square integrable functions on a quantum configuration

\footnotetext{
3 The analysis of [9] is motivated by Sahlmann's pioneering work [7].

4 It turns out that the considerations of sections III VI do not depend on the fine details of the asymptotic conditions. The imposition of gauge and diffeomorphism invariance and the definition of linear and angular momenta in section VII and VIII do depend on detailed asymptotic conditions.
} 
space which is a topological completion of the classical space of connections. Every classical connection $A$ is 'smooth' (more precisely, finitely differentiable) and satisfies conditions appropriate to asymptotic flatness and thereby defines a homomorphism from the space of background electric field labels to $U(1)$ through the background exponential function $\beta_{\bar{E}}(A)=\exp \left(i \int_{\Sigma} \bar{E}_{i}^{a} A_{a}^{i}\right)$. Thus any classical connection can be thought of as a homomorphism from the space of all $\bar{E}$ 's to $U(1)$ which is (a) well defined and (b) functionally differentiable with respect to $\bar{E}$. Property (a) is a result of the fall-off behaviour of $A$ and the 'dual' fall-off behaviour of $\bar{E}^{5}$ and property (b) is a consequence of the differential properties of $A, \bar{E}$. We show that any element of the quantum configuration space defines a homomorphism which satisfies property (a) but not necessarily property (b). We argue that the satisfaction of property (a) implies that the quantum connections satisfy a weakened form of the classical fall-off conditions on the connection.

The detailed content of sections IV to VI is as follows. In section [V] we prove a key Master Lemma which establishes that given a finite set of independent probes and a corresponding set of elements in $S U(2)$ (one for each independent edge) and $U(1)$ (one for each rationally independent electric field), there exists a classical connection satisfying the asymptotic conditions such that the evaluation of the relevant set of holonomies and background exponentials on this connection reproduces the given set of group elements to arbitrary accuracy. The Master Lemma is used extensively in sections $\mathrm{V}$ and VI. In section $\mathrm{V}$ we complete the Abelian Poisson bracket algebra of holonomies and background exponentials, $\mathcal{H B A}$, to the $C^{*}$ algebra, $\overline{\mathcal{H B A}}$ and identify its spectrum $\Delta$ with the quantum configuration space of generalised connections. We show that each element of the spectrum corresponds to a pair of homomorphisms, one homomorphism from the path groupoid to $S U(2)$ and the other from the Abelian group of electric fields to $U(1)$. The first homomorphism corresponds to the algebraic structure provided by the holonomies and the second to that provided by the background exponentials. We argue that the existence of this second homomorphism and the satisfaction of asymptotic conditions by every element of the Abelian group of electric fields imply the imposition of a weakened version of the classical asymptotic boundary conditions on elements of the spectrum. Next, we justify the identification of the spectrum as the quantum configuration space by showing that the KS Hilbert space is isomorphic to the space $L^{2}\left(\Delta, d \mu_{\mathrm{KS}}\right)$ of square integrable functions on the spectrum with respect to a suitably defined measure $d \mu_{\mathrm{KS}}$. We do this by showing that the expectation value function with respect to any KS spin net state of section II defines a positive linear function on $\overline{\mathcal{H B} A}$. The GNS construction together with standard Gel'fand theory then provides an isomorphism between the action of the holonomy and background exponential operators in the KS representation and their action by multiplication on $L^{2}\left(\Delta, d \mu_{\mathrm{KS}}\right)$. Finally, we show that the electric flux operator is represented as an operator on $L^{2}\left(\Delta, d \mu_{\mathrm{KS}}\right)$ and that this representation completes the isomorphism between the KS representation of section II and the $L^{2}\left(\Delta, d \mu_{\mathrm{KS}}\right)$ representation. In section $\mathrm{VI}$ we show that the spectrum $\Delta$ is homeomorphic to an appropriate projective limit space $\mathcal{A}$ whose fundamental building blocks are products of finite copies of $S U(2)$ and $U(1)$ and that the measure $d \mu_{\mathrm{KS}}$ can be realised as a projective limit measure of the Haar measure on these building blocks.

Section VII is devoted to a discussion of gauge transformations and symmetries of the classical theory. We describe the group of gauge transformations, Aut, of $S U(2)$ rotations and diffeomorphisms connected to identity and subject to appropriate asymptotic behaviour.

\footnotetext{
${ }^{5}$ By this we mean that $\bar{E}$ has a fall off behaviour which ensures the well definedness of $\int_{\Sigma} \bar{E}_{i}^{a} A_{a}^{i}$.
} 
Next, we describe the group Aut ${ }^{\stackrel{\circ}{E}}$ of asymptotic symmetries which are connected to identity and show that Aut is a normal subgroup of Aut ${ }^{\circledR}$. Finally we show that the quotient of Aut $^{\stackrel{\circ}{E}}$ by Aut is the semidirect product of the group of asymptotic translations with that of asymptotic rotations so that $\mathrm{Aut}^{\stackrel{\circ}{E}} / \mathrm{Aut}=\mathbb{R}^{3} \rtimes S U(2)$. In section VIII we show that the groups Aut and Aut ${ }^{\circ}$ (suitably restricted to the semianalytic category) are unitarily implemented on the KS Hilbert space. We construct a large sector of gauge invariant states by averaging over the action of the gauge group Aut (suitably restricted to the semianalytic category) and show that this sector supports a unitary representation of Aut ${ }^{\stackrel{\circ}{*}} / \mathrm{Aut}$. We show that, while for trivial topology, Aut ${ }^{\stackrel{\circ}{E}} /$ Aut acts effectively as $\mathbb{R}^{3} \rtimes S O(3)$, for the nontrivial topologies considered by Friedman and Sorkin [14], gauge invariant states exist which display fermionic behaviour under $2 \pi$ rotations so that Aut ${ }^{\stackrel{\Xi}{E}} /$ Aut is represented non-trivially as $\mathbb{R}^{3} \rtimes S U(2) .{ }^{6}$ Thus our work yields a fully rigorous implementation of the beautiful "Spin $\frac{1}{2}$ from Gravity" behaviour predicted by Friedman and Sorkin. We close section VIII with a brief discussion of the construction of eigenstates of angular and spatial momenta. Section IX contains our concluding remarks. A number of technicalities are relegated to the Appendices.

Before we proceed to the next section, we reiterate that the results of sections IVF VI represent simple generalizations of results obtained in the spatially compact case in Reference [13. Hence, in our presentation, we shall endeavour to employ the notation of [13] wherever possible and we shall be explicit only in aspects of argumentation which differ from those contained in Reference [13]. Considerable parts of our argumentation will be identical to those in Reference [13] and for such parts we shall simply refer the reader to that work. Similar remarks apply to our exposition in section VIII of this paper in relation to the contents of Reference [9]. We use units such that $c=8 \pi \gamma G=\hbar=1$ where $\gamma$ is the Barbero-Immirzi parameter.

\section{CLASSICAL PRELIMINARIES}

\section{A. Differential Structure}

Our classical departure point is a semianalytic version of the description given in [17, 19] for asymptotically flat canonical gravity. The Cauchy slice $\Sigma$ is taken to be a $C^{k}, k>>1$ semianalytic manifold without boundary admitting certain structure required for the notion of asymptotic flatness. This structure is as follows. Let $U_{0} \subset \mathbb{R}^{3}$ be the complement of the unit ball in $\mathbb{R}^{3}, U_{0}:=\left\{\left(x^{1}, x^{2}, x^{3}\right) \in \mathbb{R}^{3}:\left(x^{1}\right)^{2}+\left(x^{2}\right)^{2}+\left(x^{3}\right)^{2}>1\right\}$. We require the existence of a preferred (semianalytic) chart $\chi_{0}: U_{0} \rightarrow \Sigma$ such that $K:=\Sigma \backslash \chi_{0}\left(U_{0}\right)$ is compact. We refer to it as the Cartesian chart. In this chart we use the notation: $\vec{x}:=\left(x^{1}, x^{2}, x^{3}\right)=\left\{x^{\alpha}\right\}$, $r:=\sqrt{\left(x^{1}\right)^{2}+\left(x^{2}\right)^{2}+\left(x^{3}\right)^{2}}$ and $\hat{x}:=\vec{x} / r$. Spatial infinity is approached as $r \rightarrow \infty$.

It is important to note that while $\Sigma$ is itself $C^{k}$ and semianalytic, on $\chi_{0}\left(U_{0}\right)$ we may still define semianalytic functions on $\Sigma$ which are of arbitrary degree of differentiability with respect to the preferred Cartesian coordinates. More in detail, since $U_{0}$ is an open set in $\mathbb{R}^{3}$, we can define semianalytic $C^{p}$ functions, even for $p>k$, with respect to this preferred $\mathbb{R}^{3}$ structure. Such functions are still $C^{k}$ semianalytic functions on $\Sigma$; they just happen to possess higher differentiability with respect to the preferred Cartesian coordinates. We

\footnotetext{
${ }^{6}$ See [15] for a discussion of Friedman and Sorkin ideas in LQG.
} 
shall refer to this degree of differentiability in the preferred Cartesian chart as the Cartesian degree of differentiability. Next, we detail two examples of such functions which we shall use in this paper.

First note that the discussion above, together with the definition of semianalyticity [22], implies that a semianalytic $C^{k}$ function of Cartesian degree of differentiability $p=\infty$ is an analytic function of the Cartesian coordinates in $\chi_{0}\left(U_{0}\right)$. An example of such a function is one which is $C^{k}$ and semianalytic on $\Sigma \backslash \chi_{0}\left(U_{0}\right)$ and which coincides with the function $r^{-1}$ in $\chi_{0}\left(U_{0}\right)$. Since we are away from $r=0$, the function $r^{-1}$ is analytic in the Cartesian chart so that the function in question has Cartesian degree of differentiability $p=\infty$. A similar conclusion holds for functions which agree asymptotically with the function $r$.

Another example of the functions which we shall use in this work is provided in Appendix A wherein we show that any $C^{p}$ semianalytic function of $\hat{x}$ naturally defines a semianalytic function of Cartesian degree of differentiability $p$ on $\chi_{0}\left(U_{0}\right)$. This implies that, for example, any semianalytic $C^{k}$ function on $\Sigma \backslash \chi_{0}\left(U_{0}\right)$ which agrees with such a function on $\chi_{0}\left(U_{0}\right)$ for $p \geq k$ is $C^{k}$ semianalytic on $\Sigma$.

To summarise: If we restrict attention to the behaviour of functions in $\chi_{0}\left(U_{0}\right) \subset \Sigma$, then semianalytic functions of Cartesian differentiability $p$ are seminalytic $C^{k}$ functions on $\chi_{0}\left(U_{0}\right) \subset \Sigma$ for $p \geq k$ else they are seminalytic $C^{p}$ functions on $\chi_{0}\left(U_{0}\right) \subset \Sigma$.

Next, we introduce the notion of asymptotic fall offs of functions of Cartesian differentiability $p$. These fall offs refer to the behaviour of the function in $\chi_{0}\left(U_{0}\right)$ for large enough $r$. We shall say that a function of Cartesian differentiability $C^{p}$ is of order $O\left(r^{-\beta}\right)$ if for sufficiently large $r$ and $m=0, \ldots, p$ the $m$-th partial derivatives with respect to the preferred Cartesian coordinates are bounded by $\mathrm{cr}^{-\beta-m}$ for some constant $c$ independent of $m$.

The notions of Cartesian degree of differentiability together with that of fall-off order can be applied to tensor fields on $\Sigma$ as follows. The maximum degree of differentiability of tensor fields on (the $C^{k}$ semianalytic manifold) $\Sigma$ is $C^{k-1}$. Components of tensor fields in $\chi_{0}\left(U_{0}\right) \subset \Sigma$ can be evaluated in the preferred Cartesian chart. Each such component can be viewed as a function on $\chi_{0}\left(U_{0}\right) \subset \Sigma$. This function in turn can be a linear combination of functions each of different Cartesian degree of differentiability $p$ and associated order $O\left(r^{-\beta}\right)$. Indeed, this is the typical situation we shall encounter in this paper.

Note that $\Sigma$ can of course also be given the less restrictive structure of a $C^{k}$ manifold by completing its maximal semianalytic atlas to a $C^{k}$ one. In this setting $\Sigma$ is still the disjoint union of $\Sigma \backslash \chi_{0}\left(U_{0}\right)$ and its asymptotic region $\chi_{0}\left(U_{0}\right)$ and the asymptotic region is still equipped with the preferred Cartesian chart described in the first paragraph of this section. It is then easy to see that the notions of Cartesian degree of differentiability as well as fall off order continue to be well defined.

In summary, $\Sigma$ can be thought of as follows. $\Sigma$ is a $C^{k}$ manifold. On $\Sigma$ there is a preferred family of $C^{k}$ charts which endow $\Sigma$ with the structure of a $C^{k}$ semianalytic manifold, with this preferred family comprising a maximal semianalytic atlas on $\Sigma$. In this family of semianalytic charts, one of the charts is the Cartesian chart on $\chi_{0}\left(U_{0}\right)$. From this point of view $\Sigma$ has the ability to support, simultaneously, both finite differentiability structures as well as the more restricted semianalytic structures. This is the point of view we shall employ in the rest of this work. In general, as in the case of compact space LQG [29], classical structures will be of finite differentiability but not necessarily semianalytic whereas structures relevant to quantum theory will be chosen to be semianalytic. 


\section{B. Phase space variables and functions}

In this section we relax the condition of semianalyticity and work with $\Sigma$ as a $C^{k}$ asymptotically flat manifold

The phase space of interest is coordinatized by pairs $\left(A_{a}^{i}, E_{i}^{a}\right)$ of $s u(2)$-valued 1-forms and densitized vector fields satisfying certain fall off conditions at infinity. Here $i=1,2,3$ are internal indices with respect to a fixed $s u(2)$ basis $\tau_{i}$ satisfying $\left[\tau_{i}, \tau_{j}\right]=\epsilon_{i j k} \tau_{k}$ (we work on a fixed global trivialization of the bundle). $A_{a}^{i}, E_{i}^{a}$ are taken to be respectively $C^{k-2}, C^{k-1}$ (not necessarily semianalytic) tensors on $\Sigma .{ }^{7}$ Let $\stackrel{\circ}{E}_{i}^{a}$ be a fixed 'zeroth order' triad such that in the Cartesian chart $\stackrel{\circ}{E}_{i}^{\alpha}=\delta_{i}^{\alpha}$. The conditions on $\left(A_{\alpha}^{i}, E_{i}^{\alpha}\right), i, \alpha=1,2,3$ (where $\alpha$ refers to components in the preferred Cartesian chart $\left.\left\{x^{\alpha}\right\}\right)$ as $r \rightarrow \infty$ are taken to be:

$$
\begin{aligned}
& E_{i}^{\alpha}=\stackrel{\circ}{E}_{i}^{\alpha}+\frac{h_{i}^{\alpha}(\hat{x})}{r}+O\left(r^{-1-\epsilon}\right), \\
& A_{\alpha}^{i}=\frac{g_{\alpha}^{i}(\hat{x})}{r^{2}}+O\left(r^{-2-\epsilon}\right)
\end{aligned}
$$

where $0<\epsilon<1,{ }^{8}$ and $h_{i}^{\alpha}, g_{\alpha}^{i}$ are functions on the Cartesian sphere satisfying:

$$
\begin{aligned}
h_{i}^{\alpha}(-\hat{x}) & =h_{i}^{\alpha}(\hat{x}), \\
g_{\alpha}^{i}(-\hat{x}) & =-g_{\alpha}^{i}(\hat{x}) .
\end{aligned}
$$

For technical reasons (see for instance footnote 32 we require $h_{i}^{\alpha}, g_{\alpha}^{i}$ to be respectively $C^{k}, C^{k-1}$ as functions on the sphere. As indicated in the previous section, these specifications are consistent with the degree of differentiability of the tensor fields $E_{i}^{a}$ and $A_{a}^{i}$ on $\Sigma$ and correspond to the ' $1 / r$ ' and ' $1 / r^{2}$ ' parts of the triad and connection fields being of Cartesian degree of differentiability $k$ and $k-1$ respectively. We denote by $\mathcal{E}_{E}$ the space of $s u(2)$-valued electric fields satisfying $(2.1)$ and $\mathcal{A}$ the space of $s u(2)$-valued one forms satisfying (2.2). As in $\mathrm{LQG}, \mathcal{A}$ plays the role of classical configuration space.

Conditions (2.1), (2.2), (2.3), (2.4) are motivated by the parity conditions of references [17, 19] and, indeed, at first glance look identical to them. However, whereas the standard parity conditions are defined in the $C^{\infty}$ setting, the parity conditions (2.1), (2.2) are defined for finitely differentiable fields.

The conditions ensure the symplectic structure

$$
\Omega\left(\delta_{1}, \delta_{2}\right):=\int_{\Sigma}\left(\delta_{1} A_{a}^{i} \delta_{2} E_{i}^{a}-\delta_{2} A_{a}^{i} \delta_{1} E_{i}^{a}\right)
$$

is well defined. The elementary phase space functions which admit direct operator corre-

\footnotetext{
${ }^{7}$ We take $E^{a}$ to be of maximal differentiability $\left(C^{k-1}\right)$. The reason we take the connection to be $C^{k-2}$ is that in classical theory $A_{a}$ arises as a combination of extrinsic curvature and spin connection, both of which involve first derivatives of the triad.

8 The condition $\epsilon<1$ plays no restriction (for $\epsilon_{0}>\epsilon, f(x)=O\left(r^{-\epsilon_{0}}\right) \Longrightarrow f(x)=O\left(r^{-\epsilon}\right)$ ). Its purpose is to allow for simplifications of the type: $O\left(r^{-1-\epsilon}\right)+O\left(r^{-2}\right)=O\left(r^{-1-\epsilon}\right)$.
} 
spondents in the KS representation are given by

$$
\begin{aligned}
h_{e}(A) & :=\mathcal{P} e^{\int_{e} A}, \\
\beta_{\bar{E}}(A) & :=e^{i \int_{\Sigma} \operatorname{Tr}\left[\bar{E}^{a} A_{a}\right]} \\
F_{S, f}(E) & :=\int_{S} d S_{a} \operatorname{Tr}\left[f E^{a}\right] .
\end{aligned}
$$

Here $h_{e}(A)$ is the $S U(2)$ matrix valued holonomy of the connection along the compactly supported edge $e, \beta_{\bar{E}}(A)$ is the background exponential labelled by the background electric field $\bar{E}$ subject to appropriate asymptotic conditions, and $F_{S, f}(E)$ is the electric flux smeared with the $s u(2)$-valued function $f$ through the surface $S \subset \Sigma$. "Tr" stands for -2 times the standard matrix trace, $\operatorname{Tr}[M]:=-2\left(M_{11}+M_{22}\right)$, so that $\operatorname{Tr}\left[\tau_{i} \tau_{j}\right]=\delta_{i j}$. Anticipating the key role that the 'probes' $e, \bar{E}, S, f$ play in quantum theory, we shall choose them to be semianalytic. The detailed definition of $e$ and the asymptotic conditions on $\bar{E}$ are displayed in section IIC. The detailed choice of surfaces $S$ and associated smearing functions $f$ is displayed in section IID.

\section{Probes of the connection}

\section{Holonomy related structures}

An oriented piecewise semianalytic curve $c$ is defined as a piecewise semianalytic map $c:[0,1] \rightarrow \Sigma$ (see Definition 6.2 .1 of [20]). The compact semianalytic parameter range $[0,1]$ in the definition of $c$ implies that curves are compactly supported in $\Sigma$ and, hence, do not extend to spatial infinity. Paths are defined by identifying curves differing in orientation preserving reparametrizations and retracings. They form a groupoid $\mathcal{P}$ with composition law given by concatenation. The compact support of the curves $c$ and the definition of paths in terms of equivalence classes of finite compositions of curves implies that any path $p$ is compactly supported in $\Sigma$. An edge $e$ is a path $p$ that has a representative curve such that the image $\tilde{e}$ of this representative curve is a connected 1 dimensional semianalytic submanifold with 2 point boundary. Thus, edges are elementary paths which generate $\mathcal{P}$ by composition. We denote by $\operatorname{Hom}(\mathcal{P}, S U(2))$ the set of all homomorphisms from $\mathcal{P}$ to $S U(2)$. The set of holonomies $\left\{h_{p}[A], p \in \mathcal{P}\right\}$ for a given connection $A \in \mathcal{A}$ define an element in $\operatorname{Hom}(\mathcal{P}, S U(2))$ by virtue of the composition law: $h_{p^{\prime}}[A]_{C}^{D}=h_{p^{\prime}}[A]_{C}^{C^{\prime}} h_{p}[A]_{C^{\prime}}^{D}$, whenever the endpoint $f(p)$ of $p$ coincides with the beginning point $b\left(p^{\prime}\right)$ of $p^{\prime}$.

A set of edges $e_{1}, \ldots, e_{n}$ is said to be independent if their intersections can only occur at their endpoints, i.e. if $\tilde{e}_{i} \cap \tilde{e}_{j} \subset\left\{b\left(e_{i}\right), b\left(e_{j}\right), f\left(e_{i}\right), f\left(e_{j}\right)\right\}$. We denote by $\gamma:=\left(e_{1}, \ldots, e_{n}\right)$ an ordered set of independent edges and by $\mathcal{L}_{\mathrm{H}}$ the set of all such ordered sets of independent edges. Given $\gamma, \gamma^{\prime} \in \mathcal{L}_{\mathrm{H}}$, we say that $\gamma^{\prime} \geq \gamma$ iff all edges of $\gamma$ can we written as composition of edges (or their inverses) of $\gamma^{\prime}$. With this relation $\left(\mathcal{L}_{\mathrm{H}}, \geq\right)$ becomes a directed set [13].

To summarise: The compactness of the curves $c$ ensures that no subtelities ensue relative to the treatment of the compact case in [13]. 


\section{Background exponential related structures}

The probes associated to the background exponentials are electric fields. Each such electric field $\bar{E}$ satisfies the fall offs:

$$
\bar{E}_{i}^{\alpha}=\frac{\bar{h}_{i}^{\alpha}(\hat{x})}{r}+O\left(r^{-1-\epsilon}\right)
$$

with

$$
\bar{h}_{i}^{\alpha}(-\hat{x})=\bar{h}_{i}^{\alpha}(\hat{x})
$$

where, as before, the expressions refer to components in the (semianalytic) Cartesian chart $\left\{x^{\alpha}\right\}, \bar{E}^{a}$ is $C^{k-1}$ semianalytic and $\bar{h}_{i}^{\alpha}$ is $C^{k}$ semianalytic as a function on the sphere. Note that these conditions, modulo semianalyticity, correspond to those associated with variations of asymptotically flat triads (2.1) and that these conditions ensure convergence of the 3-dimensional integral in (2.7).

We denote by $\mathcal{E}$ the set of all such electric fields $\bar{E}_{i}^{a} . \mathcal{E}$ is an Abelian group with composition law given by addition. We denote by $\operatorname{Hom}(\mathcal{E}, U(1))$ the set of homomorphism from $\mathcal{E}$ to $U(1)$. The set of background exponentials $\left\{\beta_{\bar{E}}[A], \bar{E} \in \mathcal{E}\right\}$ for a given connection $A \in \mathcal{A}$ define an element in $\operatorname{Hom}(\mathcal{E}, U(1))$ by virtue of $\beta_{\bar{E}^{\prime}}[A] \beta_{\bar{E}}[A]=\beta_{\bar{E}^{\prime}+\bar{E}}[A]$.

A set of electric fields $\bar{E}_{1}, \ldots, \bar{E}_{N}$ is said to be independent, if they are algebraically independent, i.e. if they are independent under linear combinations with integer coefficients:

$$
\sum_{I=1}^{N} q_{I} \bar{E}_{I}=0, q_{I} \in \mathbb{Z} \Longleftrightarrow q_{I}=0, I=1, \ldots, N .
$$

We denote by $\Upsilon=\left(\bar{E}_{1}, \ldots, \bar{E}_{N}\right)$ an ordered set of independent electric fields. The set of all ordered sets of independent electric fields is denoted by $\mathcal{L}_{\mathrm{B}}$. Given $\Upsilon, \Upsilon^{\prime} \in \mathcal{L}_{\mathrm{B}}$ we say that $\Upsilon^{\prime} \geq \Upsilon$ iff all the electric fields in $\Upsilon$ can be written as algebraic combinations of those in $\Upsilon^{\prime}$. One can verify that $\left(\mathcal{L}_{\mathrm{B}}, \geq\right)$ is a directed set exactly as done in [13] (the proof is purely algebraic and insensitive to the detailed properties of $\mathcal{E}$ such as the particular fall-off (2.9)).

\section{Combined Holonomy and Background exponential structures}

The combined set of labels associated to holonomies and background exponentials is given by pairs $l=(\gamma, \Upsilon) \in \mathcal{L}_{\mathrm{H}} \times \mathcal{L}_{\mathrm{B}}=: \mathcal{L}$ with preorder relation given by $\left(\gamma^{\prime}, \Upsilon^{\prime}\right) \geq$ $(\gamma, \Upsilon)$ iff $\gamma^{\prime} \geq \gamma$ and $\Upsilon^{\prime} \geq \Upsilon$. It immediately follows that $\mathcal{L}$ is a directed set. Given $l=\left(e_{1}, \ldots, e_{n}, \bar{E}_{1}, \ldots, \bar{E}_{N}\right) \in \mathcal{L}$ we define the group

$$
G_{l}:=S U(2)^{n} \times U(1)^{N}
$$

and the map

$$
\begin{aligned}
\pi_{l}: \mathcal{A} & \rightarrow G_{l} \\
A & \mapsto \pi_{l}[A]:=\left(h_{e_{1}}[A], \ldots, h_{e_{n}}[A], \beta_{\bar{E}_{1}}[A], \ldots, \beta_{\bar{E}_{N}}[A]\right) .
\end{aligned}
$$

It will be useful for later purposes to describe the relation between $\pi_{l}$ and $\pi_{l^{\prime}}$ whenever $l^{\prime} \geq l$.

Let $l^{\prime} \geq l$. Edges $e_{i} \in l$ can then be written as compositions of edges in $l^{\prime}$. Let us denote 
this relation by: $e_{i}=\tilde{p}_{i}\left(e_{1}^{\prime}, \ldots\right)$, where $\tilde{p}_{i}$ denotes a particular composition of edges (and their inverses) in $l^{\prime}$. This corresponds to a relation on the holonomies of the form:

$$
h_{e_{i}}[A]=h_{\tilde{p}_{i}\left(e_{1}^{\prime}, \ldots\right)}[A]=p_{i}\left(h_{e_{1}^{\prime}}[A], \ldots\right),
$$

where $p_{i}: S U(2)^{n^{\prime}} \rightarrow S U(2)$ is the map determined by interpreting the compositions rules of $\tilde{p}_{i}$ as matrix multiplications. For example, if $e_{1}=e_{2}^{\prime} \circ\left(e_{1}^{\prime}\right)^{-1}$ then $p_{1}\left(g_{1}^{\prime}, \ldots, g_{n^{\prime}}^{\prime}\right)=g_{2}^{\prime}\left(g_{1}^{\prime}\right)^{-1}$. Similarly, electric fields $\bar{E}_{I} \in l$ can be written as integer linear combinations of electric fields in $l^{\prime}$ :

$$
\bar{E}_{I}=\tilde{P}_{I}\left(\bar{E}_{1}^{\prime}, \ldots\right):=\sum_{J=1}^{N^{\prime}} q_{I}^{J} \bar{E}_{J}^{\prime}, \quad q_{I}^{J} \in \mathbb{Z}, \quad I=1, \ldots, N .
$$

Associated to 2.16 there is the map $P_{I}: U(1)^{N^{\prime}} \rightarrow U(1)$ given by $P_{I}\left(u_{1}^{\prime}, \ldots, u_{N^{\prime}}^{\prime}\right)=$ $\Pi_{J=1}^{N^{\prime}}\left(u_{J}^{\prime}\right)^{q_{I}^{J}}$ so that

$$
\beta_{\bar{E}_{I}}[A]=\beta_{\tilde{P}_{I}\left(\bar{E}_{1}^{\prime}, \ldots\right)}[A]=P_{I}\left(\beta_{\bar{E}_{1}^{\prime}}[A], \ldots\right) .
$$

The above maps combine in a map

$$
p_{l, l^{\prime}}:=\left(p_{1}, \ldots, p_{n}, P_{1}, \ldots, P_{N}\right): G_{l^{\prime}} \rightarrow G_{l}
$$

in terms of which equations (2.15) and (2.17) are summarized as:

$$
\pi_{l}[A]=p_{l, l^{\prime}}\left(\pi_{l^{\prime}}[A]\right)
$$

expressing $\pi_{l}$ in terms of $\pi_{l^{\prime}}$ and $p_{l, l^{\prime}}$.

\section{Probes of the Electric field}

The probes of the electric field are the pairs $(S, f)$ which go into the construction of the electric flux $F_{S, f}$ of equation (2.8). We choose the surfaces $S$ to be oriented compact 2 dimensional semianalytic submanifolds of $\Sigma$ with or without boundary. For $S$ without boundary, we require the $s u(2)$ valued function $f$ to be semianalytic as a function on $S$. For $S$ with boundary, we require that $f$ be semianalytic and compactly supported as a function on the interior, Int $S$, of $S$. The choice of surfaces ensures that any such surface $S$ intersects any edge (as defined in section II C) in at most a finite number of connected semianalytic submanifolds (i.e. in a finite number of isolated points and/or a finite number semianalytic edges tangential to $S$ ). This in turns ensures that the Poisson bracket between the holonomies and fluxes, is, as in the standard LQG case, well defined. The restriction of the support of $f$ within a compact set of Int $S$ is to avoid any further technicalties associated with semianalyticity in the presence of boundaries.

\section{E. Poisson brackets between Elementary functions}

The classical Poisson brackets of the above functions is as follows. Holonomies and background exponentials Poisson commute among themselves and each other. The Abelian Poisson bracket algebra they generate plays a crucial role in the construction of the quantum configuration space to be described in later sections. Poisson brackets between fluxes and 
holonomies are exactly as in LQG, since our choice of edges and surfaces is such that they do not 'reach' infinity and, as in the semianalytic, compact $\Sigma$ case, they can only intersect each other finitely many times. The Poisson bracket between fluxes and background exponentials is

$$
\left\{\beta_{\bar{E}}, F_{S, f}\right\}=i F_{S, f}(\bar{E})
$$

where $F_{S, f}(\bar{E})=\int_{S} d S_{a} \operatorname{Tr}\left[f \bar{E}^{a}\right]$. In order to obtain a classical algebra involving brackets between fluxes, it would be necessary to repeat the analysis of Reference [13] which, following References [21, 22] identifies these Poisson brackets with commutators of derivations on the space of connection dependent functions generated by sums and products of holonomies and background exponentials. While we anticipate no obstruction to doing so, we leave this for future work.

\section{THE KS REPRESENTATION FOR THE ASYMPTOTICALLY FLAT CASE}

In section III A we display the KS Hilbert space representation for the asymptotically flat case. Section IIIB discusses the implementation of the classical asymptotic boundary conditions on the electric field in this representation.

\section{A. The KS Hilbert space and operator actions thereon.}

The KS Hilbert space, $\mathcal{H}_{\mathrm{KS}}$, is spanned by states of the form $|s, E\rangle$, where $s$ is an LQG spin network with edges as described in the previous section, and $E^{a}$ a semianalytic asymptotically flat background electric field satisfying (2.1). The inner product is given by

$$
\left\langle s^{\prime}, E^{\prime} \mid s, E\right\rangle=\left\langle s \mid s^{\prime}\right\rangle_{\mathrm{LQG}} \delta_{E^{\prime}, E},
$$

where $\left\langle s \mid s^{\prime}\right\rangle_{\mathrm{LQG}}$ is the LQG spin network inner product and $\delta_{E^{\prime}, E}$ the Kronecker delta.

Holonomies 2.6) and background exponentials (2.7) act by

$$
\begin{aligned}
\hat{h}_{e}{ }_{D}^{C}|s, E\rangle & =\left|\hat{h}_{e}^{\mathrm{LQG} A}{ }_{B} s, E\right\rangle, \\
\hat{\beta}_{\bar{E}}|s, E\rangle & =|s, E+\bar{E}\rangle .
\end{aligned}
$$

Above, we have used the notation of [12] wherein given an LQG operator $\hat{O}$ with action $\hat{O}|s\rangle=\sum_{I} O_{I}^{(s)}\left|s_{I}\right\rangle$ in standard LQG, we have defined the state $|\hat{O} s, E\rangle$ in the KS representation through

$$
|\hat{O} s, E\rangle:=\sum_{I} O_{I}^{(s)}\left|s_{I}, E\right\rangle .
$$

Note that the action 3.3 is well defined because the new background field state label $E+\bar{E}$ satsifies the boundary conditions $(2.1)$ by virtue of the boundary conditions $\sqrt{2.9}$ on $\bar{E} .^{9}$

\footnotetext{
${ }^{9}$ It is in this sense that the imposition of the asymptotic conditions 2.1 on the background electric field labels of states is consistent with the imposition of the asymptotic conditions 2.9) on the background electric field labels of operators (see the remarks in the main text of section 1 preceding Footnote 2 .
} 
The action of fluxes is given by

$$
\hat{F}_{S, f}|s, E\rangle=\left|\hat{F}_{S, f}^{\mathrm{LQG}} s, E\right\rangle+F_{S, f}(E)|s, E\rangle,
$$

where $f=f^{i} \tau_{i}$ is the $s u(2)$-valued smearing scalar on the surface $S$ and $F_{S, f}(E)=$ $\int_{S} d S_{a} f^{i} E_{i}^{a}$ the flux associated to the background electric field $E_{i}^{a}$.

It is easily verified that with these definitions the background exponentials act as unitary operators with $\left(\hat{\beta}_{\bar{E}}\right)^{\dagger}=\hat{\beta}_{-\bar{E}}$, the fluxes as self adjoint (or, more precisely, symmetric) operators, and that adjointness relations of holonomy operators reproduce the relations of classical holonomies under complex conjugation.

The (unitary) action of spatial diffeomorphisms and $S U(2)$ gauge transformations will be discussed in section VIII.

\section{B. Implementation of the electric field boundary conditions.}

From a phase space perspective, the classical boundary conditions described in section IIB can be cast in the following form: Given a phase space point $(A, E)$, there exists a radius $r_{(A, E)}$ in the asymptotic region $\Sigma \backslash K$ such that Eqns. (2.1), (2.2) hold for $r>r_{(A, E)}$. By $r$ greater than a given radius $r_{0}$ we mean points in $\Sigma$ lying in:

$$
\Sigma \backslash \Sigma_{r_{0}}:=\left\{\vec{x} \in \mathbb{R}^{3}:\left(x^{1}\right)^{2}+\left(x^{2}\right)^{2}+\left(x^{3}\right)^{2}>r_{0}^{2}\right\}
$$

where (3.6) is described in the Cartesian chart. $\Sigma_{r_{0}}$, defined as the complement of the set (3.6), represents the points of $\Sigma$ 'inside' the 2-sphere of radius $r_{0}$.

An analogue statement in quantum theory (regarding the electric field boundary conditions) is: Given a KS spinnet $|\psi\rangle \equiv|s, E\rangle$, there exists a radius $r_{\psi}$ in the asymptotic region such that (i) the spin network $s$ lies in $\Sigma_{r_{\psi}}$ (as defined above), (ii) $E$ satisfies (2.1) for $r>r_{\psi}$. Property (i), ensured by the compactness of $s$, implies that the spinnet $|\psi\rangle$ can be thought of as an eigenvector of an electric field operator $\hat{E}^{a}(x)$ with $x$ outside $\Sigma_{r_{\psi}}$ :

$$
\hat{E}^{a}(x)|\psi\rangle=E^{a}(x)|\psi\rangle, \quad x \in \Sigma \backslash \Sigma_{r_{\psi}} .
$$

From property (ii), the eigenvalue in (3.7) satisfies (2.1). Thus Eq. (3.7) represents a quantum version of the electric field boundary condition (2.1).

An alternative strategy more attuned to standard LQG would be to describe the electric field boundary conditions purely in terms of fluxes. This is however more involved as it requires the use of surfaces approaching infinity. In appendix B we present the first steps towards the implementation of this idea.

\section{THE MASTER LEMMA}

\section{A. Statement of the Lemma}

Let $\left(e_{1}, \ldots, e_{n}\right)$ be a set of $n$ independent edges. Let $\left(\bar{E}_{1}, \ldots, \bar{E}_{N}\right)$ be a set of $N$ algebraically independent electric fields, each with asymptotic behaviour (2.9). Define the group

$$
G:=S U(2)^{n} \times U(1)^{N}
$$


and the map

$$
\begin{aligned}
\pi: \mathcal{A} & \rightarrow G \\
A & \mapsto \pi[A]:=\left(h_{e_{1}}[A], \ldots, h_{e_{n}}[A], \beta_{\bar{E}_{1}}[A], \ldots, \beta_{\bar{E}_{N}}[A]\right) .
\end{aligned}
$$

Then the map $\pi$ has dense range in $G$.

To prove the lemma, it clearly suffices to construct $A^{g, \delta} \in \mathcal{A}$ such that, given $g:=$ $\left(g_{1}, \ldots, g_{n}, u_{1}, \ldots, u_{N}\right) \in G_{l}=S U(2)^{n} \times U(1)^{N}$ and any $\delta>0, A^{g, \delta}$ has the property that

$$
\begin{aligned}
\left|h_{e_{\alpha}}\left[A^{g, \delta}\right]_{C}^{D}-g_{\alpha C}\right| & \leq C_{1} \delta \forall \alpha=1, . ., n \text { and } C, D=1,2 . \\
\left|\beta_{\bar{E}_{I}}\left[A^{g, \delta}\right]-e^{i \theta_{I}}\right| & \leq C_{2} \delta \forall I=1, . ., N .
\end{aligned}
$$

Here $C_{1}, C_{2}$ are $\delta$ - independent constants, $C, D$ are $S U(2)$ matrix indices and $u_{I}=: e^{i \theta_{I}}, \theta_{I} \in$ $\mathbb{R}$.

The proof parallels the proof of the Master Lemma for the case of compact $\Sigma$ in [13]. It consists of five steps, of which only the first one requires a minor adaptation to the present, non-compact $\Sigma$ case. We outline the steps in section IV B below referring to [13] for technical proofs. The end result is a connection $A^{g, \delta} \in \mathcal{A}$ which implements equations (4.4) and (4.5) and which falls off faster than $r^{-2}$ near spatial infinity thus satisfying the asymptotic conditions (2.2) with no leading order $r^{-2}$ term.

We remark here that connections with only subleading behaviour at infinity cannot describe spacetime solutions with non-vanishing linear momenta since the surface terms corresponding to the evaluation of the linear momenta only involve the leading order $r^{-2}$ part of the connection. Note however that we have only shown that a finite number of arbitrarily prescribed $S U(2)$ and $U(1)$ elements can be approximated to arbitrary accuracy using such connections with no leading order asymptotic behaviour. As we show in section $\mathrm{VA}$ the set of all edge holonomies and all background exponentials seperate points in $\mathcal{A}$. This together with the Master Lemma implies that one needs infinitely many evaluations of holonomies and background exponentials to distinguish between connections with vanishing and nonvanishing leading order fall offs.

As a final remark, note that by setting $l=\left(e_{1}, \ldots, e_{n}, \bar{E}_{1}, \ldots, \bar{E}_{N}\right)$, the Master Lemma implies that the map $\pi_{l}$ of equation $(2.14)$ is dense in $G_{l}$ for any $l$.

\section{B. Steps in the proof of the Lemma}

(i) Construct a connection $\bar{A}^{B, \delta}$ which satisfies 4.5 :

Assume without loss of generality that the electric fields $\left\{\bar{E}_{1}, \ldots, \bar{E}_{N}\right\}$ are ordered so that the first $M$ are linearly independent and that the remaining $P:=N-M$ electric fields can be written as linear combinations of the first $M$ ones so that:

$$
\bar{E}_{M+j}=\sum_{\mu=1}^{m} k_{j}^{\mu} \bar{E}_{\mu}, \quad j=1, \ldots, P,
$$


for some real constants $k_{j}^{\mu}$. Next, we construct $M$ connections $A^{\nu}, \nu=1, \ldots, M$ satisfying:

$$
\int_{\Sigma} \operatorname{Tr}\left[\bar{E}_{\mu}^{a} A_{a}^{\nu}\right]=\delta_{\mu}^{\nu}, \quad \mu, \nu=1, \ldots, M
$$

An adapted version of the argument given in [13] for the existence of such connections is as follows. Let $q_{a b}$ be an asymptotically flat metric in $\Sigma$ so that $q_{a b}=\stackrel{\circ}{q}_{a b}+O(1 / r)$ with $\stackrel{\circ}{q}_{a b}$ being the fixed flat metric at infinity. Let $\Omega_{p}$ be a strictly positive function on $\Sigma$ with an $O\left(r^{p}\right)$ behavior at infinity. Define the metric $q_{a b}^{(p)}:=\Omega_{p}^{2} q_{a b}$ so that we have

$$
q_{a b}^{(p)}:=\Omega_{p}^{2} q_{a b}, \quad q_{a b}^{(p)}=O\left(r^{2 p}\right) \text { as } r \rightarrow \infty .
$$

Define the following bilinear form on $\mathcal{E}$ :

$$
\left\langle\bar{E}, \bar{E}^{\prime}\right\rangle:=\int_{\Sigma} q^{-1 / 2} q_{a b} \operatorname{Tr}\left[\bar{E}^{a} \bar{E}^{\prime b}\right] .
$$

Let us now set $p>1$. Then the asymptotic behavior of $\Omega_{p}$ implies the integral $(4.9)$ is convergent. Nondegeneracy of $q_{a b}$ and positivity of $\Omega_{p}$ implies $\langle$,$\rangle is positive definite. It$ then follows that the $M \times M$ matrix defined by: $\left\langle\bar{E}_{\mu}, \bar{E}_{\nu}\right\rangle, \mu, \nu=1, \ldots, M$ is invertible. Denote its inverse by $c_{\mu \nu}$. Then the one-forms $A_{a}^{\nu}:=q^{-1 / 2} \sum_{\rho=1}^{M} c_{\rho \nu} q_{a b} \bar{E}_{\rho}^{b}$ satisfy (4.7) and, from equation (4.8), fall off as $r^{-p-1}$ at infinity so that $A_{a}^{\nu} \in \mathcal{A}$. By exactly the same steps as in [13], one can then find $M$ real numbers $t_{\mu}^{(\delta)}, 1 \leq \mu \leq M$ such that

$$
\bar{A}_{a}^{B, \delta}:=\sum_{\mu=1}^{M} t_{\mu}^{(\delta)} A_{a}^{\mu}
$$

satisfies 4.5 with $C_{2}=1$.

(ii) For sufficiently small $\epsilon$ and appropriately chosen $\epsilon$ - independent charts, construct balls $B_{\alpha}(2 \epsilon), \alpha=1, . ., n$ of coordinate size $2 \epsilon$ such that

$$
\begin{array}{r}
B_{\alpha}(2 \epsilon) \cap B_{\beta}(2 \epsilon)=\emptyset \text { iff } \alpha \neq \beta \\
B_{\alpha}(2 \epsilon) \cap \tilde{e}_{\beta}=\emptyset \text { iff } \alpha \neq \beta \\
\bar{B}_{\alpha}(2 \epsilon) \cap \tilde{e}_{\alpha} \text { is a semianalytic edge }
\end{array}
$$

Since the edges are compactly supported, the construction of (ii) is identical to the one given in [13].

(iii) Construct a real semianalytic function $f_{\epsilon}$ such that $\left|f_{\epsilon}\right| \leq 1$ on $\Sigma$ with

$$
\begin{aligned}
f_{\epsilon} & =1 \text { on } \Sigma-\cup_{\alpha} B_{\alpha}(2 \epsilon) \\
& =0 \text { on } \cup_{\alpha} B_{\alpha}(\epsilon),
\end{aligned}
$$

where $B_{\alpha}(\epsilon)$ denotes the $\epsilon$ size ball with the same centre as $B_{\alpha}(2 \epsilon)$.

The construction of $f_{\epsilon}$ is identical to that in [13].

(iv) From 4.13 it follows that each $e_{\alpha}$ can be written as the composition of three edges 
$s_{\alpha}^{1}, s_{\alpha}^{2}, s_{\alpha}$,

$$
e_{\alpha}=s_{\alpha}^{1} \circ s_{\alpha} \circ s_{\alpha}^{2}
$$

with

$$
\begin{aligned}
\tilde{s}_{\alpha} & :=\tilde{e}_{\alpha} \cap \bar{B}_{\alpha}(\epsilon) \\
\tilde{s}_{\alpha}^{1} \cup \tilde{s}_{\alpha}^{2} & =\tilde{e}_{\alpha} \cap\left(\Sigma-B_{\alpha}(\epsilon)\right) .
\end{aligned}
$$

Define:

$$
h_{s_{\alpha}^{i}}\left[A^{B, f}\right]=: g_{\alpha}^{i}, \quad i=1,2
$$

where $A^{B, f}:=f_{\epsilon} \bar{A}^{B, \delta}$. and construct a connection $A^{\epsilon}$ supported in $\cup_{\alpha} B_{\alpha}(\epsilon)$ such that

$$
h_{s_{\alpha}}\left[A^{\epsilon}\right]=\left(g_{\alpha}^{1}\right)^{-1} g_{\alpha}\left(g_{\alpha}^{2}\right)^{-1} .
$$

The construction of $A^{\epsilon}$ is identical to that in [13].

(v) Set $A^{g, \delta}:=A^{B, f}+A^{\epsilon}$. It is then possible to show that for small enough $\epsilon, A^{g, \delta}$ satisfies equations (4.4) and (4.5) with $C_{1}=0$ and $C_{2}=2$. The proof of this assertion is identical to that in [13].

\section{THE QUANTUM CONFIGURATION SPACE}

In section $\mathrm{VA}$ we introduce the (Abelian) holonomy-background exponential algebra $\mathcal{H B} \mathcal{A}$ and its associated $C^{*}$ algebra $\overline{\mathcal{H B A}}$. The spectrum $\Delta$ of $\overline{\mathcal{H B A}}$ is shown to be a topological completion of the space of connections $\mathcal{A}$. In section $\mathrm{VB}$ we give a characterization of $\Delta$ in terms of certain homomorphisms, to be later used in section $\mathrm{VE}$. In section $\mathrm{VC}$ we realize $\mathcal{H}_{\mathrm{KS}}$ as an $L^{2}$ space over $\Delta$ (with an integration measure $d \mu_{\mathrm{KS}}$ ) and show that the holonomies and background exponentials act by multiplication in this realization. Section $\mathrm{VD}$ is concerned with the action of flux operators on $L^{2}\left(\Delta, d \mu_{\mathrm{KS}}\right)$. We note that while the general arguementation of sections $\mathrm{VC}$ and $\mathrm{VD}$ follows that for the spatially compact case in [13], a key difference from the compact case stems from the unavailability of the "| $s=0, E=0\rangle "$ cyclic state of [13] in the asymptotically flat context due to the incompatibility of the $E=0$ label with asymptotic flatness. Notwithstanding this the considerations of sections $\mathrm{VC}$ and $\mathrm{VD}$ establish the unitary equivalence of the standard KS representation of the holonomy, background exponential and flux operators with their representations on $L^{2}\left(\Delta, d \mu_{\mathrm{KS}}\right)$. In section $\mathrm{VE}$ we use the characterization of section $\mathrm{VB}$ to show that elements of $\Delta$ satisfy a weakened version of the asymptotic conditions satisfied by elements of $\mathcal{A}$.

\section{A. The algebras $\mathcal{H B A}$ and $\overline{\mathcal{H B A}}$}

We denote by $\mathcal{H B} \mathcal{A}$ the $*$-algebra of functions of $\mathcal{A}$ generated by the elementary functions (2.6) and (2.7), with * relation given by complex conjugation. By the same arguments as those given in [13], any element $a[A] \in \mathcal{H B} \mathcal{A}$ can be written as

$$
a[A]=a_{l}\left(\pi_{l}[A]\right)
$$


for some $l=\left(e_{1}, \ldots, e_{n}, \bar{E}_{1}, \ldots, \bar{E}_{N}\right) \in \mathcal{L}, \pi_{l}[A]$ as in 2.14 $)$, and $a_{l} \in \operatorname{Pol}\left(G_{l}\right)$, where $\operatorname{Pol}\left(G_{l}\right)$ is the set of functions on $G_{l}$ that depend polynomially on the $S U(2)$ and $U(1)$ entries and the complex conjugates of these entries. Given $a, l, a_{l}$ for which (5.1) holds, $a$ is said to be compatible with $l, l$ is said to be compatible with $a$ and $a, l$ are said to be mutually compatible. That a given mutually compatible pair $a, l$ uniquely specifies $a_{l} \in \operatorname{Pol}\left(G_{l}\right)$ follows from the continuity of $a_{l}$ together with the denseness of $\pi_{l}[\mathcal{A}]$ in $G_{l}$. If $l^{\prime} \geq l$ and $l$ is compatible with $a$, then $l^{\prime}$ is compatible with $a$ and

$$
a_{l^{\prime}}=a_{l} \circ p_{l, l^{\prime}},
$$

with $p_{l, l^{\prime}}: G_{l^{\prime}} \rightarrow G_{l}$ the map induced by the way probes in $l$ are written in terms of probes in $l^{\prime}$ as described in section IIC 3 (see Reference [13] for a proof of this claim).

On $\mathcal{H} \mathcal{B A}$ we define the norm:

$$
\|a\|:=\sup _{A \in \mathcal{A}}|a[A]|, \quad a \in \mathcal{H B} \mathcal{A} .
$$

When $a$ is written as in (5.1), the lemma of section IV] implies:

$$
\|a\|=\sup _{A \in \mathcal{A}}\left|a_{l}\left(\pi_{l}[A]\right)\right|=\sup _{g \in G_{l}}\left|a_{l}(g)\right| .
$$

Being a sup norm, (5.3) is compatible with the product and complex-conjugation star relations on $\mathcal{H B A}$. The completion $\overline{\mathcal{H B A}}$ of $\mathcal{H B} \mathcal{A}$ in the norm $(5.3)$ is then a unital $C^{*}$ algebra. We denote the Gel'fand spectrum of $\overline{\mathcal{H B A}}$ by $\Delta . \quad \Delta$ is a compact, Hausdorff space and $\overline{\mathcal{H B A}} \simeq C(\Delta)$ where $C(\Delta)$ is the $C^{*}$ algebra of continuous functions on $\Delta$. We denote by $\operatorname{Cyl}(\Delta) \subset C(\Delta)$ the subalgebra of continuous functions corresponding to $\mathcal{H B A}$ in the Gel'fand identification.

From [23], the fact that $\mathcal{H B} \mathcal{A}$ separates points in $\mathcal{A}$, implies that $\mathcal{A}$ is topologically dense in $\Delta$ or, equivalently, that $\Delta$ is the completion of $\mathcal{A}$ in the Gel'fand topology. To see that $\mathcal{H B} \mathcal{A}$ separates points in $\mathcal{A}$, we proceed as follows. Given $A_{a}^{\prime} \neq A_{a}^{\prime \prime} \in \mathcal{A}$ we want to find $a \in \mathcal{H B A}$ such that $a\left[A^{\prime}\right] \neq a\left[A^{\prime \prime}\right]$. It is enough to consider elements of the form $a=\beta_{\bar{E}}$. Setting $A_{a}:=A_{a}^{\prime}-A_{a}^{\prime \prime}$ the condition translates to show that for any $A_{a} \neq 0$ there exists $\bar{E}^{a} \in \mathcal{E}$ such that $\beta_{\bar{E}}[A] \neq 1$. Since $A_{a} \neq 0$ we can find a local chart where at least one of its components, say $A_{1}^{1}$, is nonzero. Further, we can find a small enough open ball $U$ where this component is of definite sign, say $\left.A_{1}^{1}\right|_{U}>0$. By using semianalytic bump functions similar to those used in step (iii) of the master Lemma (see Eq. (A7) of [13]) one can construct an electric field $\bar{E}^{a} \in \mathcal{E}$ with support in $U$ such that $\left.\bar{E}_{1}^{1}\right|_{U}>0$ and with all remaining components being zero. Then $s:=\int_{\Sigma} \bar{E}_{i}^{a} A_{a}^{i}=\int_{U} \bar{E}_{1}^{1} A_{1}^{1}>0$. One can further take $\bar{E}^{a}$ such that $s \notin 2 \pi \mathbb{Z}$ so that $\beta_{\bar{E}}[A]=e^{i s} \neq 1$.

\section{B. Characterization of the spectrum $\Delta$}

Recall from II C1 and II C2, that $\operatorname{Hom}(\mathcal{P}, S U(2))$ and $\operatorname{Hom}(\mathcal{E}, U(1))$ denote the space of homomorphisms from the groupoid of paths $\mathcal{P}$ to the group $S U(2)$ and from the Abelian group of electric fields $\mathcal{E}$ to the group $U(1)$. As in the case of compact $\Sigma$, the spectrum admits a characterization in terms of these homomorphisms. More in detail, each element of the spectrum is in unique correspondence with a pair of homomorphisms, one member of 
the pair in $\operatorname{Hom}(\mathcal{P}, S U(2))$ and the other in $\operatorname{Hom}(\mathcal{E}, U(1))$ i.e. there is a bijection between $\Delta$ and $\operatorname{Hom}(\mathcal{P}, S U(2)) \times \operatorname{Hom}(\mathcal{E}, U(1))$. As in the compact $\Sigma$ case [13], we construct this bijection as follows.

First recall from standard Gel'fand theory that elements of the spectrum are in correspondence with $C^{*}$ algebraic homomorphisms from $\overline{\mathcal{H B A}}$ to $\mathbb{C}$. Accordingly, given such a homomorphism $\phi \in \Delta$ define:

$$
\begin{aligned}
s_{\phi}: \mathcal{P} & \rightarrow S U(2) \\
p & \mapsto s_{\phi}(p)_{C}^{D}:=\phi\left(h_{p}{ }_{C}^{D}\right)
\end{aligned}
$$

and

$$
\begin{aligned}
u_{\phi}: & \mathcal{E} \\
& \rightarrow U(1) \\
\bar{E} & \mapsto u_{\phi}(\bar{E}):=\phi\left(\beta_{\bar{E}}\right) .
\end{aligned}
$$

As in [13], one can verify that the $*$-homomorphism properties of $\phi$ imply that $s_{\phi} \in$ $\operatorname{Hom}(\mathcal{P}, S U(2))$ and $u_{\phi} \in \operatorname{Hom}(\mathcal{E}, U(1))$. Conversely, given $s \in \operatorname{Hom}(\mathcal{P}, S U(2))$ and $u \in \operatorname{Hom}(\mathcal{E}, U(1))$ one can find $\phi \in \Delta$ such that $u_{\phi}=u$ and $s_{\phi}=s$ as follows. $\phi$ is first defined as a $*$-homomorphism from $\mathcal{H B A}$ to $\mathbb{C}$ by:

$$
\phi(a):=a_{l}\left(s\left(e_{1}\right), \ldots, s\left(e_{n}\right), u\left(\bar{E}_{1}\right), \ldots, u\left(\bar{E}_{N}\right)\right),
$$

where $l=\left(e_{1}, \ldots, e_{n}, \bar{E}_{1}, \ldots, \bar{E}_{N}\right)$ is compatible with $a$. From the homomorphism properties of $s$ and $u$, and the considerations of section $\mathrm{VA}$ one can verify that $(5.9)$ is independent of the choice of $l$ and satisfies the $*$-homomorphism properties [13]. Finally one can show boundedness of $\phi$ by means of Eq. (5.4), so that $\phi$ admits a unique extension to $\overline{\mathcal{H B A}}[13]$. Thus $\phi \in \Delta$, and by construction $u_{\phi}=u$ and $s_{\phi}=s$.

\section{Realization of the KS Hilbert space as the space $L^{2}\left(\Delta, d \mu_{\mathrm{KS}}\right)$}

Fix a KS state $|0, E\rangle$ where $E^{a}$ is an asymptotically flat electric field. We wish to define a positive linear functional (PLF) on $\mathcal{H B A}$ associated to this state. ${ }^{10}$ Given $a \in \mathcal{H B A}$ and $l \in \mathcal{L}$ compatible with $a$, let $\hat{a}_{l}$ be the operator on the KS Hilbert space associated to $a_{l}$. Define:

$$
\omega(a):=\left\langle 0, E\left|\hat{a}_{l}\right| 0, E\right\rangle=\int_{G_{l}} a_{l}(g) d \mu_{l},
$$

where $d \mu_{l}$ is the Haar measure on the group $G_{l}$ normalized so that $\int_{G_{l}} d \mu_{l}=1$. The second equality in (5.10) is described in appendix B.2 of [13. That this definition is independent of the choice of compatible label $l \in \mathcal{L}$ follows from the 'cylindrical consistency' of the measures $\left\{\mu_{l}, l \in \mathcal{L}\right\}$, as shown in appendix C.3 of [13]. Positivity is also easily verified from the integral representation in $(5.10)$.

${ }^{10}$ Since we have not shown that the operators $3.2,3.3$ induce a representation of $\mathcal{H B} \mathcal{A}$ on the KS Hilbert space, we here verify explicitly that expectation values yield a well defined PLF on $\mathcal{H B A}$. 
From the master lemma and Eq. (5.1) one finds $\omega$ is bounded:

$$
|\omega(a)|=\left|\int_{G_{l}} a_{l}(g) d \mu_{l}\right| \leq \sup _{g \in G_{l}}\left|a_{l}(g)\right|=\|a\| .
$$

Eq. (5.11) serves two purposes. On the one hand it tell us that $a[A]=0 \Longrightarrow \omega(a)=0$, so that $\omega$ is well defined on the abstract algebra $\mathcal{H B} \mathcal{A}$ (i.e. it respects all quotienting relations like $\beta_{\bar{E}_{1}} \beta_{\bar{E}_{2}}-\beta_{\bar{E}_{1}+\bar{E}_{2}}=0$ ). On the other hand, the boundedness property implies the existence of a unique extension of $\omega$ to $\overline{\mathcal{H B} \mathcal{B}} \simeq C(\Delta)$ [20]. It then follows by Riesz-Markov theorem that $\omega$ defines a regular measure $\mu_{\mathrm{KS}}$ on $\Delta$ satisfying:

$$
\omega(a)=\int_{\Delta} a d \mu_{\mathrm{KS}}
$$

where in the RHS of $(5.12) a$ is seen as an element of $C(\Delta)$ via the standard Gel'fand identification.

We now show that $\mathcal{H}_{\mathrm{KS}} \simeq L^{2}\left(\Delta, d \mu_{\mathrm{KS}}\right)$ as Hilbert space representations of $\overline{\mathcal{H} \mathcal{B} \mathcal{A}}$. Note that $|0, E\rangle \in \mathcal{H}_{\mathrm{KS}}$ is a cyclic state i.e. the action of $\mathcal{H} \mathcal{B} \mathcal{A}$ on $|0, E\rangle$ yields a dense subspace $\mathcal{D}_{\mathrm{KS}}$ in $\mathcal{H}_{\mathrm{KS}} \cdot{ }^{11}$ Next, choose an element $\Psi_{0} \in C(\Delta)$ such that

$$
\left|\Psi_{0}\right|^{2}=1 \text { on } \Delta
$$

Define the vector space $\mathcal{D}_{C(\Delta)}:=\left\{a \Psi_{0} \in C(\Delta), a \in \mathcal{H B} \mathcal{A} \subset C(\Delta)\right\}$ and equip $\mathcal{D}_{C(\Delta)}$ with the inner product

$$
\left(a \Psi_{0}, b \Psi_{0}\right)=\int_{\Delta}\left(a \Psi_{0}\right)^{*}\left(b \Psi_{0}\right) d \mu_{\mathrm{KS}}, \quad a, b \in \mathcal{H B} \mathcal{A}
$$

Define $V_{E, \Psi_{0}}: \mathcal{D}_{\mathrm{KS}} \rightarrow \mathcal{D}_{C(\Delta)}$ by $V_{E, \Psi_{0}}(\hat{a}|0, E\rangle):=a \Psi_{0}$ where $\hat{a}$ denotes the KS operator correspondent of the element $a \in \mathcal{H B} \mathcal{A}$ and $a \Psi_{0} \in C(\Delta)$ as in equation (5.14) above. Then it is straightforward to check using equations (5.13), (5.14) and (5.12) that $V_{E, \Psi_{0}}$ is a one to one map from $\mathcal{D}_{\mathrm{KS}}$ to $\mathcal{D}_{C(\Delta)}$ which preserves the inner product. It follows that $V_{E, \Psi_{0}}$ admits a unique extension from $\mathcal{H}_{\mathrm{KS}}$ to $L^{2}\left(\Delta, d \mu_{\mathrm{KS}}\right)$. From the fact that $\mathcal{H B} \mathcal{A}$ is commutative, it follows that $V_{E, \Psi_{0}}$ unitarily maps the KS representation of $\mathcal{H B} \mathcal{A}$ to a representation by multiplication on $L^{2}\left(\Delta, d \mu_{\mathrm{KS}}\right)$ by the continuous functions in $\mathcal{H B} \mathcal{A} \subset C(\Delta)$. Since the holonomies and background exponentials are represented as bounded operators in the KS representation, and since elements of $\mathcal{H B \mathcal { A }}$ are finite polynomials in these basic operators, it follows that $\mathcal{H B} \mathcal{A}$ is represented as an algebra of bounded operators on $\mathcal{H}_{\mathrm{KS}}$. This boundedness is readily seen in the representation on $L^{2}\left(\Delta, d \mu_{\mathrm{KS}}\right)$ as a consequence of the boundedness of continuous functions on the compact Haussdorff space $\Delta$.

Finally, note that $L^{2}\left(\Delta, d \mu_{\mathrm{KS}}\right)$ supports not only the operator algebra $\mathcal{H B} \mathcal{A}$ but also its $C^{*}$ completion, $\overline{\mathcal{H B A}}$. Once again, since continuous functions on compact Haussdorff space are bounded, it follows that elements of $\overline{\mathcal{H B A}}$ are represented as bounded operators on

11 The action of a background exponential changes the label $E$ of $|0, E\rangle$ to any desired background field state label and appropriate finite sums and products of holonomy operators change the label 0 of $|0, E\rangle$ to any desired LQG spin net label. It follows the dense set of the finite span of KS spinnets may be obtained by the action of operators in $\mathcal{H B A}$. 
$L^{2}\left(\Delta, d \mu_{\mathrm{KS}}\right)$. It follows by using the inverse map $V_{E, \Psi_{0}}^{-1}$ that we may define the action of elements of $\overline{\mathcal{H B A}}$ on $\mathcal{H}_{\mathrm{KS}}$ also as bounded operators on $\mathcal{H}_{\mathrm{KS}}$. This completes the demonstration that $\mathcal{H}_{\mathrm{KS}} \equiv L^{2}\left(\Delta, d \mu_{\mathrm{KS}}\right)$ as Hilbert space representations of $\overline{\mathcal{H} \mathcal{B} \mathcal{A}}$.

Next we note that the identification of $\mathcal{H}_{\mathrm{KS}}$ with $L^{2}\left(\Delta, d \mu_{\mathrm{KS}}\right)$ is not unique. The nonuniqueness is two fold. First, as seen above, the map $V_{E, \Psi_{0}}$ may be defined for any $\Psi_{0} \in$ $C(\Delta)$ subject to equation (5.13). Second, it is easy to check that the PLF $\omega_{E}$ of equation (5.10) is independent of the choice of $E$ i.e. for any $a \in \mathcal{H B} \mathcal{A}$ and any $E_{1}, E_{2}$ subject to the asymptotic conditions (2.1) $\omega_{E_{1}}(a)=\omega_{E_{2}}(a)$. Thus the unitary map $V_{E, \Psi_{0}}$ may be defined for any $E$ subject to (2.1) and any $\Psi_{0} \in C(\Delta)$. In the compact case, there is a natural choice, namely $E=0$ and $\Psi_{0}=1$ and this together with the natural action of flux operators as derivations yields the unitary equivalence of $L^{2}\left(\Delta, d \mu_{\mathrm{KS}}\right)$ and $\mathcal{H}_{\mathrm{KS}}$ as Hilbert space representations of the holonomy, background exponential and flux operators [13.

In contrast, in the asymptotically flat case, there is no such natural choice because $E=0$ is not consistent with the requirements of asymptotic flatness (2.1). In order to proceed further, we make some choice, $E$, consistent with (2.1) and, henceforth, set $\Psi_{0}=1$ and use the notation $V_{E}:=V_{E, \Psi_{0}=1}$. As we shall see in the next section, the unitary equivalence of the KS representation of holonomies and background exponentials with the one on $L^{2}\left(\Delta, d \mu_{\mathrm{KS}}\right)$ defined by $V_{E}$ can be extended to include the action of flux operators. While, in contrast to the action of operators in $\overline{\mathcal{H B A}}$, the explicit action of the flux operators on (a dense subspace of $L^{2}\left(\Delta, d \mu_{\mathrm{KS}}\right)$ will involve the choice of $E$, the extension will be seen to hold for any choice of $E$ subject to (2.1).

\section{Action of Fluxes on $L^{2}\left(\Delta, d \mu_{\mathrm{KS}}\right)$}

The discussion of section $\mathrm{VC}$ indicates that elements $a \in \overline{\mathcal{H \mathcal { B }}} \simeq C(\Delta)$ have a dual interpretation: When seen as elements of $C(\Delta)$ they are 'wavefunctions' in the $L^{2}$ representation, i.e. vectors in the Hilbert space; when seen as elements of $\overline{\mathcal{H B} \mathcal{A}}$, they are naturally associated with operators $\hat{a}$ on the Hilbert space $\mathcal{H}_{\mathrm{KS}}$. Accordingly, when we use the former interpretation we shall refer to $a$ as a wavefunction and when we use the latter we shall denote $a$ by $\hat{a}$ and refer to it as an operator.

Let us fix a 'reference' KS spinet $|0, E\rangle$ as before. The wavefunction associated to a KS spinnet $|s, \tilde{E}\rangle$ via the map $V_{E}$ is then given by: $T_{s} \beta_{\tilde{E}-E} \in \operatorname{Cyl}(\Delta)$, where $T_{s}[A] \in \mathcal{H B} \mathcal{A}$ is the spin network function associated to $s[20], \beta_{\tilde{E}-E}[A] \in \mathcal{H B} \mathcal{A}$ the background exponential function (2.7), and $T_{s}, \beta_{\tilde{E}-E}$ the respective elements in $\operatorname{Cyl}(\Delta)$ under the Gel'fand identification $\mathcal{H} \mathcal{B} \mathcal{A} \simeq \operatorname{Cyl}(\Delta)$.

From the action of the flux operator $\hat{F}_{S, f}$ on $\mathrm{KS}$ spinnets $(3.5)$, and the above, $E$ dependent identification of $\mathrm{KS}$ spinnets with elements of $\operatorname{Cyl}(\Delta)$, we obtain the following action of the fluxes in the latter space:

$$
\hat{F}_{S, f}^{(E)}\left(T_{s} \beta_{\bar{E}}\right)=\left(\hat{F}_{S, f}^{\mathrm{LQG}} T_{s}\right) \beta_{\bar{E}}+F_{S, f}(E+\bar{E}) T_{s} \beta_{\bar{E}} .
$$

As indicated in section $\mathrm{VC}$, even though the natural choice $E=0$ is not available if we wish to describe asymptotically flat geometries, all different choices of asymptotically

\footnotetext{
12 Notice that there are no elements in $\operatorname{Cyl}(\Delta)$ that could correspond to " $\beta_{\tilde{E}}$ " or " $\beta_{E}$ " since these labels do not satisfy the required fall off 2.9 . However $\tilde{E}-E \in \mathcal{E}$ is a valid label for the background exponentials.
} 
flat 'reference' $E$ yield unitarily equivalent Hilbert spaces. The unitary transformation that maps the wave functions in the " $E^{\prime}$ representation" associated with $V_{E^{\prime}}$ to those in the " $E$ representation" associated with $V_{E}$ is given by the multiplication operator:

$$
\begin{aligned}
\hat{U}_{E, E^{\prime}}: L^{2}\left(\Delta, d \mu_{\mathrm{KS}}\right) & \rightarrow L^{2}\left(\Delta, d \mu_{\mathrm{KS}}\right) \\
\Psi & \mapsto \beta_{E^{\prime}-E} \Psi .
\end{aligned}
$$

The multiplicative operators from $\mathcal{H B} \mathcal{A}$ remain unaltered by this action, whereas flux operators (5.15) in the $E^{\prime}$ representation are mapped into the corresponding flux operators in the $E$ representation:

$$
\hat{U}_{E, E^{\prime}} \hat{F}_{S, f}^{\left(E^{\prime}\right)} \hat{U}_{E^{\prime}, E}=\hat{F}_{S, f}^{(E)}
$$

as can be verified by straightforward computation.

\section{E. Asymptotic behaviour of elements of $\Delta$.}

From section $\mathrm{VA}$, the quantum configuration space $\Delta$ is a topological completion of the classical configuration space of connections $\mathcal{A}$. Since elements of $\mathcal{A}$ are subject to the asymptotic conditions (2.2), one may interpret this feature of $\Delta$ as evidence for the incorporation of $(2.2)$ in the quantum theory. However, as we note in section VI, while topologically dense, $\mathcal{A}$ is measure theoretically sparse in $\Delta$ with respect to the measure $\mu_{K S}$. Since expectation value computations in quantum theory do not obtain contributions from zero measure sets, if there exists a measure theoretically thick set in $\Delta$ whose elements can be thought of as blatantly violating the conditions $(2.2)$, we would conclude that these conditions do not leave an imprint in quantum theory. That this is not so follows from the fact that every element of $\Delta$ satisfies a quantum analog these conditions. This quantum analog can be thought of as weaker than the classical conditions (2.2). To see this we proceed as follows.

From section $\mathrm{VB}$, every element of the quantum configuration space $\Delta$ is identified with an element of $\operatorname{Hom}(\mathcal{E}, U(1))$. In particular since $\mathcal{A} \subset \Delta$, every $A \in \mathcal{A}$ defines $h_{A} \in \operatorname{Hom}(\mathcal{E}, U(1))$ through $h_{A}(\bar{E})=\beta_{\bar{E}}(A)=e^{i \int_{\Sigma} A_{a}^{i} \bar{E}_{i}^{a}} \in U(1)$. The asymptotic fall offs on $A \sqrt{2.2}$ ensure that the integral $\int_{\Sigma} A_{a}^{i} \bar{E}_{i}^{a}$ is well defined for every $\bar{E} \in \mathcal{E}$ by virtue of the boundary conditions 2.9 on elements of $\mathcal{E}$. This in turn ensures the well- definedness of $h_{A}$. However, given the conditions (2.9), the conditions (2.2) are only sufficient (rather than necessary) for the well definedness of this integral. For example, consider a connection A with asymptotic behaviour

$$
\mathbf{A}_{\alpha}^{i}=\frac{d f}{d r} \frac{g_{\alpha}^{i}(\hat{x})}{r^{2}}+\frac{C_{\alpha}^{i}}{(r \log r)^{2}}
$$

with $f(r)=\cos \left(e^{r}\right)$ and $C_{\alpha}^{i}$ constant. A by parts integration shows that the contribution of the first term to the integral $\int_{\Sigma} \mathbf{A}_{a}^{k} \bar{E}_{k}^{a}$ (with $\bar{E} \in \mathcal{E}$ ) is finite and it is easy to check the finiteness of the contribution of the second term. Clearly $\mathbf{A} \notin \mathcal{A}$. Nevertheless, $h_{\mathbf{A}} \in \operatorname{Hom}(\mathcal{E}, U(1))$ with $h_{\mathbf{A}}(\bar{E})=\beta_{\bar{E}}(\mathbf{A})=e^{i \int_{\Sigma} \mathbf{A}_{a}^{i} \bar{E}_{i}^{a}} \in U(1)$. Since elements of $\Delta$ include all homomorphisms in $\operatorname{Hom}(\mathcal{E}, U(1))$ such connections are also a part of the quantum configuration space. Thus, instead of the detailed fall offs (2.2), such quantum connections $A^{\prime}$ 
satisfy the weaker condition that the integral:

$$
\int_{\Sigma} A_{b}^{\prime i} \bar{E}_{i}^{b}<\infty \quad \forall \bar{E} \in \mathcal{E}
$$

Of course not every homomorphism arises from a connection field; just as in the spatially compact case, there may be no connection field associated with a given homomorphism. To see this, note that the homomorphisms defined by elements of $\mathcal{A}$ have a 'smooth' dependence with respect to the background field which allows the computation of the functional derivative of any such homomorphism with respect to its background field dependence. More in detail, $h_{A} \in \operatorname{Hom}(\mathcal{E}, U(1))$ is defined through $h_{A}(\bar{E} \in \mathcal{E})=\exp \left(i \int A_{a}^{i} \bar{E}_{i}^{a}\right)$ so that

$\frac{\delta h_{A}}{\delta \bar{E}(x)}=i A(x) h_{A}$. However, it is possible to define elements of $\operatorname{Hom}(\mathcal{E}, U(1))$ which are not functionally differentiable. ${ }^{13}$

For such 'distributional' elements of $\Delta$ which cannot be realised as a connection field, a quantum analogue of condition $(5.20)$ is provided by the defining property of elements of $\Delta$ : namely that every such element yields a well defined element of $\operatorname{Hom}(\mathcal{E}, U(1))$. It is in this sense that elements of the quantum configuration space satisfy a weakened version of the classical fall offs 2.2 .

Note that the quantum configuration space is an enlargement of the classical one in two different ways. First, as in the compact case, the elements of $\Delta$ may be 'distributional' in the sense that the homomorphisms they define are not functionally differentiable with respect to their electric field argument. Second, elements of $\Delta$ satisfy the (distributional) analog of the weaker boundary condition $(5.20)$ than $(2.2)$. The weakening of the functional differentiability property may be thought of as indicative of a certain 'blindness' of (distributional) elements of $\Delta$ to the differential structure of the manifold (the differential structure is, after all, what allows the definition of infinitesimal variations of $\bar{E}$ and the consequent computation of the functional derivative. We speculate in closing that the weakened asymptotic behaviour may similarly be thought of as an insensitivity to the function space structure underlying the infinite dimensional symplectic structure of the classical phase space. More in detail, one expects that pairs of elements, one in the space of variations of the classical triad field and one in (the tangent space to) $\mathcal{A}$ lie in the tangent space to the infinite dimensional classical phase space. From this point of view the condition (2.2) and the (variation of the) condition (2.1) could, in a more rigorous treatment, possibly be seen as linked to the function space topology of the phase space. The weakened condition (5.20) is too coarse to define typical function space topologies and in this sense the weakened asymptotic behaviour of elements of $\Delta$ could be seen to arise from their insensitivity to the detailed function space structure.

\section{THE QUANTUM CONFIGURATION SPACE AS A PROJECTIVE LIMIT}

In section II C 3 we defined the label set $\mathcal{L}$, the spaces $\left\{G_{l}, l \in \mathcal{L}\right\}$, and the maps $\left\{p_{l, l^{\prime}}\right.$ : $\left.G_{l^{\prime}} \rightarrow G_{l}, l^{\prime} \geq l \in \mathcal{L}\right\}$. It is easy to verify that if $l^{\prime \prime} \geq l^{\prime} \geq l$ then $p_{l, l^{\prime \prime}}=p_{l, l^{\prime}} \circ p_{l^{\prime}, l^{\prime \prime}}$. Further, by the same argument as given in [13], the maps $p_{l, l^{\prime}}$ can be shown to be surjective. The

${ }^{13}$ As an example consider $A \in \mathcal{A}$ and $\phi \in \operatorname{Hom}(\mathbb{R}, U(1))$ such that $\phi(t)$ is nowhere differentiable in $\mathbb{R}$. Then $\bar{E} \rightarrow \phi\left(\int A_{a}^{i} \bar{E}_{i}^{a}\right)$ is a non-functionally differentiable element of $\operatorname{Hom}(\mathcal{E}, U(1))$. 
projective limit space $\overline{\mathcal{A}}$ can then be constructed from the family $\left(\mathcal{L},\left\{G_{l}\right\},\left\{p_{l l^{\prime}}\right\}\right)$ as in $[28]$ as follows.

One first considers the space $\mathcal{A}_{\infty}:=\times_{l \in \mathcal{L}} G_{l}$. A point in $\mathcal{A}_{\infty}$ is then given by a collection of points $\left\{x_{l} \in G_{l}\right\}$ for every $l \in \mathcal{L}$. $\mathcal{A}_{\infty}$ is given the so-called Tychonov topology [20], i.e. the weakest topology such that the canonical projections

$$
\begin{aligned}
p_{l}^{\infty}: & \mathcal{A}_{\infty} \rightarrow G_{l} \\
\left\{x_{l^{\prime}}\right\} & \mapsto x_{l}
\end{aligned}
$$

are continuous. Under this topology, $\mathcal{A}_{\infty}$ is compact and Hausdorff [20]. Next, one considers the subset of $\mathcal{A}_{\infty}$ given by:

$$
\overline{\mathcal{A}}:=\left\{\left\{x_{l}\right\} \in \mathcal{A}_{\infty} \mid p_{l, l^{\prime}}\left(x_{l^{\prime}}\right)=x_{l}, \forall l^{\prime} \geq l\right\}
$$

with the topology induced by $\mathcal{A}_{\infty}$. One can then show that $\overline{\mathcal{A}}$ is also compact and Hausdorff [28]. Finally, the projections $p_{l}: \overline{\mathcal{A}} \rightarrow G_{l}$ defined by $p_{l}:=\left.p_{l}^{\infty}\right|_{\overline{\mathcal{A}}}$ can be shown to be continuous and surjective [28].

The proof that $\overline{\mathcal{A}}$ is homeomorphic to $\Delta$ is the same as the one given in [13] following [27] and consists in showing that $C(\overline{\mathcal{A}})$ and $C(\Delta) \equiv \overline{\mathcal{H B} \mathcal{A}}$ are isomorphic as $C^{*}$ algebras. This is done by constructing a norm preserving $*$-isomorphism $T$, between $\operatorname{Cyl}(\Delta) \equiv \mathcal{H B} \mathcal{A}$ and

$$
\operatorname{Cyl}(\overline{\mathcal{A}}):=\cup_{l \in L} p_{l}^{*} \operatorname{Pol}\left(G_{l}\right) \subset C(\overline{\mathcal{A}}),
$$

given by:

$$
\begin{aligned}
T: \operatorname{Cyl}(\overline{\mathcal{A}}) & \rightarrow \mathcal{H B \mathcal { A }} \\
f=f_{l} \circ p_{l} & \mapsto T(f):=f_{l} \circ \pi_{l} .
\end{aligned}
$$

Finally, one makes use of Stone-Weierstrass theorem to show that the completion of $\operatorname{Cyl}(\overline{\mathcal{A}})$ coincides with $C(\overline{\mathcal{A}})$. Since $C(\Delta)$ can be seen as the completion of $\operatorname{Cyl}(\Delta)$, it follows that $C(\overline{\mathcal{A}})=C(\Delta)$ as $C^{*}$ algebras. By Gel'fand theory it then follows that $\overline{\mathcal{A}}$ and $\Delta$ are homeomorphic

As in [13], one can consider the two separate projective limit spaces $\overline{\mathcal{A}}_{\mathrm{H}}$ and $\overline{\mathcal{A}}_{\mathrm{B}}$ associated to $\left(\mathcal{L}_{\mathrm{H}},\left\{G_{\gamma}\right\},\left\{p_{\gamma \gamma^{\prime}}\right\}\right)$ and $\left(\mathcal{L}_{\mathrm{B}},\left\{G_{\Upsilon}\right\},\left\{p_{\Upsilon \Upsilon^{\prime}}\right\}\right)$, and show that $\overline{\mathcal{A}}=\overline{\mathcal{A}}_{\mathrm{H}} \times \overline{\mathcal{A}}_{\mathrm{B}}$. From this perspective, it may look as if one got a 'too big' space, somehow involving two copies of space of connections. Let us clarify this point. $\overline{\mathcal{A}}$ is understood as a topological completion of $\mathcal{A}$. $\mathcal{A}$ embeds in $\overline{\mathcal{A}}$ via the map $\pi_{l}$ of section II C 3 .

$$
\begin{aligned}
& \mathcal{A} \rightarrow \overline{\mathcal{A}}=\overline{\mathcal{A}}_{\mathrm{H}} \times \overline{\mathcal{A}}_{\mathrm{B}} \\
& A \mapsto\left\{\pi_{l}[A]\right\}=\left(\left\{\pi_{\gamma}[A]\right\},\left\{\pi_{\Upsilon}[A]\right\}\right)
\end{aligned}
$$

since by virtue of 2.19$)\left\{\pi_{l}[A]\right\} \in \mathcal{A}_{\infty}$ lies in $\overline{\mathcal{A}}(6.3)$. Above we also displayed the embedding in the $\overline{\mathcal{A}}_{\mathrm{H}} \times \overline{\mathcal{A}}_{\mathrm{B}}$ picture of $\overline{\mathcal{A}}$, where $\pi_{\gamma}[A]$ and $\pi_{\Upsilon}[A]$ are respectively given by the first $n$ and last $N$ components of (2.14). This product structure allows for other, inequivalent embeddings, for instance:

$$
\begin{aligned}
\mathcal{A} & \rightarrow \overline{\mathcal{A}} \\
A & \mapsto\left\{\left(\pi_{\gamma}[A], \operatorname{Id}_{G_{\Upsilon}}\right)\right\}
\end{aligned}
$$


and in this sense one could perhaps argue that $\overline{\mathcal{A}}$ is 'too big'. However the interpretation of $\overline{\mathcal{A}}$ as a completion of $\mathcal{A}$ is only with respect to the 'correct' embedding 6.8 .

The description of the measure $\mu_{\mathrm{KS}}$ on $\Delta \approx \overline{\mathcal{A}}$ can also be given in terms of cylindrically consistent measures $\left\{\mu_{l}\right\}$ on $\left\{G_{l}\right\}$ exactly as in [13], and the same argument given to show that $\mathcal{A} \subset \overline{\mathcal{A}}$ is of measure zero applies here as well.

\section{GAUGE TRANSFORMATIONS AND ASYMPTOTIC SYMMETRIES: CLASSICAL CONSIDERATIONS}

We describe the group of gauge transformations, Aut, in section VII A and the group of asymptotic symmetries, Aut ${ }^{\stackrel{\circ}{ }}$, in section VII B. The asymptotic behaviour of elements of Aut and Aut $^{E}$ displayed in sections VII A and VII B is derived as follows. We study the space of $C^{k-1} S U(2)$ rotations and $C^{k}$ diffeomorphisms subject to the restriction that their combined action preserves the space of classical triad fields with fall offs (2.1). In appendix C2 we show that this restriction on $S U(2)$ rotations and diffeomorphisms completely specifies their asymptotic behaviour. The restriction is a priori weaker than the restriction that such transformations preserve both the asymptotic fall offs on the triad as well those on the connection (2.2). However, as can be easily verified, the additional restriction of preservation of (2.2) is automatically satisfied by the transformations subject to the behaviour specified by the considerations of Appendix $\mathrm{C} 2$.

The conditions derived in Appendix $\mathrm{C} 2$ are as follows. The diffeomorphisms asymptote to a combination of rotations, translations and 'odd supertranslations' (odd supertranslations are defined in [19]). Diffeomorphisms with trivial rotational part are accompanied by $S U(2)$ transformations which asymptote to identity and diffeomorphisms with non-trivial rotational part are accompanied by $S U(2)$ transformations which compensate for this rotation so as to preserve the flat triad at infinity. Comparison with the asymptotic fall offs of the finite transformations generated by the $s u(2)$ multipliers and shift vectors which serve as smearing functions for the $S U(2)$ Gauss Law, the spatial diffeomorphism constraint and the total angular and linear momenta in the $C^{\infty}$ setting of Reference [19] allow us to identify which of the transformations of the previous paragraph are to be interpreted as gauge transformations and which as asymptotic symmetries. ${ }^{14}$ This comparison indicates we identify gauge transformations with combinations of diffeomorphisms with trivial rotational and translational parts together with $S U(2)$ transformations which asymptote to identity, and that we identify asymptotic symmetries with combinations of diffeomorphisms with non-trivial rotational and translational parts together with $S U(2)$ transformations which compensate for the rotational diffeomorphism so as to leave the fixed flat triad invariant at infinity.

In section VIIC we show that Aut is a normal subgroup of $\mathrm{Aut}^{\stackrel{\circ}{E}}$ and that the quotient group Aut ${ }^{\circ} /$ Aut is the finite dimensional group $\mathbb{R}^{3} \rtimes S U(2)$. In section VIID we display the action of semianalytic elements of Aut and $\mathrm{Aut}^{\stackrel{\circ}{E}}$ on the holonomy, flux and background exponential functions. The reason for the additional restriction of semianalyticity is that these functions are defined using semianalytic probes and the semianalyticity of these probes

${ }^{14}$ Reference 19$]$ specifies the asymptotic fall offs of the multipliers. In appendix $\mathrm{D}$ we use these conditions on the multipliers to deduce corresponding conditions on the finite $S U(2)$ transformations and diffeomorphisms generated by these multipliers. 
as well as the Poisson bracket algebra of these functions is preserved by semianalytic elements

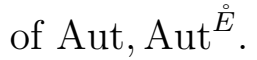

\section{A. Kinematical gauge group Aut}

A kinematic gauge transformation a consists of a pair $(g, \phi)$ where $g$ is a $C^{k-1}, S U(2)$ internal rotation, $\phi$ is a $C^{k}$ diffeomorphism and $a=(g, \phi)$ is connected to identity. ${ }^{15}$

The transformation $a \equiv(g, \phi)$ acts on phase space as:

$$
\begin{aligned}
(g, \phi) \cdot A_{a} & :=g \cdot\left(\phi \cdot A_{a}\right)=g \phi_{*} A_{a} g^{-1}-\left(\partial_{a} g\right) g^{-1}, \\
(g, \phi) \cdot E^{a} & :=g \cdot\left(\phi \cdot E^{a}\right)=g \phi_{*} E^{a} g^{-1} .
\end{aligned}
$$

$\phi_{*}$ denotes push-forward so for instance: $\left(\phi_{*} g\right)(x) \equiv g\left(\phi^{-1}(x)\right)$.

As explained in the beginning of section VII the considerations of Appendix C 2 together with the classical phase space analysis [18, 19] and the considerations of Appendix D imply the following asymptotic conditions as $r \rightarrow \infty$ on each such gauge transformation $a$ :

$$
\begin{aligned}
g(x) & =\mathbf{1}+\frac{\lambda(\hat{x})}{r}+O\left(r^{-1-\epsilon}\right), \\
\phi^{\alpha}(x) & =x^{\alpha}+s^{\alpha}(\hat{x})+O\left(r^{-\epsilon}\right),
\end{aligned}
$$

where

$$
\begin{aligned}
\lambda(-\hat{x}) & =\lambda(\hat{x}), \\
s^{\alpha}(-\hat{x}) & =-s^{\alpha}(\hat{x}),
\end{aligned}
$$

and $\lambda$ and $s^{\alpha}$ are respectively $C^{k}, C^{k+1}$ functions on the sphere. It follows from appendices $\mathrm{C} 2$ and $\mathrm{C} 1$ that the set Aut of all such gauge transformations preserves the asymptotic conditions (2.1) and (2.2). From appendix $\mathrm{C} 4$ it follows that Aut is a group. From (7.2) one finds the composition rule on Aut has the following 'semi-direct product' form:

$$
(g, \phi)\left(g^{\prime}, \phi^{\prime}\right)=\left(g \phi_{*} g^{\prime}, \phi \circ \phi^{\prime}\right)
$$

\section{B. Group Aut $^{\stackrel{\circ}{E}}$}

In the Cartesian chart $\left\{x^{\alpha}\right\}$ there is a preferred flat metric $\stackrel{\circ}{q}_{\alpha \beta}=\delta_{\alpha \beta}$ with isometries generated by (asymptotic) rotations $R_{\beta}^{\alpha} \in S O(3)$ and translations $t^{\alpha} \in \mathbb{R}^{3}$ :

$$
x^{\alpha} \rightarrow R_{\beta}^{\alpha} x^{\beta}+t^{\alpha}
$$

${ }^{15}$ We shall say that $a$ is connected to identity if there exists a one parameter family of automorphisms $a(s) \in$ Aut, $s \in[0,1]$ such that (i) $a(0)=\mathrm{Id}, a(1)=a$ (ii) for every point $p$ in $\Sigma, \phi(s)(p)=: p(s)$ is continuous with respect to the topology of $\Sigma$ and (iii) for every point $p \in \Sigma, g(s, p)$ is continuous in $S U(2)$. 
From the analysis of References [18, 19] we expect that the group of these asymptotic isometries is represented on the phase space variables $(A, E)$. We refer to this putative 'symmetry' group as Aut $\stackrel{\stackrel{\circ}{ } \text {. }}{\text {. }}$

Recall that the fall-off conditions on the electric field $E$ are such that the zeroth order value of $E$ at infinity is fixed by the triad $E^{\alpha}(2.1)$. Whereas under asymptotic translations $\stackrel{\circ}{E}^{\alpha}$ remains invariant, under asymptotic rotations it changes as

$$
\stackrel{\circ}{E}^{\alpha} \rightarrow R_{\beta}^{\alpha} \stackrel{\circ}{E}^{\beta}
$$

thus violating the fall-offs (2.1). If however the asymptotic rotation is accompanied by an internal $S U(2)$ rotation $\stackrel{\circ}{g}$ satisfying

$$
R_{\beta}^{\alpha} \stackrel{\circ}{g} \stackrel{\circ}{E}^{\beta} \stackrel{\circ}{ }-1=\stackrel{\circ}{E}^{\alpha},
$$

then the boundary condition (2.1) is preserved. This discussion suggests that elements of Aut $^{\stackrel{\circ}{E}}$ may be obtained by augmenting those in Aut with appropriate rotational and translational diffeomorphisms, together with gauge transformations of the type (7.10). Indeed as explained in the beginning of section VII this is exactly what happens.

We proceed as follows. We define every element $a \in$ Aut $^{\stackrel{\circ}{E}}$ to be in correspondence with a pair $(g, \phi)$ where $g$ is a $C^{k-1} S U(2)$ gauge transformation, $\phi$ a $C^{k}$ diffeomorphism, and $a=(g, \phi)$ is connected to identity (where by connected to identity we mean that there exists a path in Aut $^{\stackrel{\circ}{E}}$ subject to the conditions (i)- (iii) of Footnote 15). Each such element has phase space action given by (7.2). As explained in the beginning of section VII, the considerations of Appendix C 2 together with the classical phase space analysis [18, 19] and the considerations of Appendix $\mathrm{D}$ then imply the following conditions on $(g, \phi)$ as $r \rightarrow \infty$ :

$$
\begin{aligned}
g(x) & =\stackrel{\circ}{g}+\frac{\lambda(\hat{x})}{r}+O\left(r^{-1-\epsilon}\right), \\
\phi(x)^{\alpha} & =R_{\beta}^{\alpha}(\stackrel{\circ}{g}) x^{\beta}+t^{\alpha}+s^{\alpha}(\hat{x})+O\left(r^{-\epsilon}\right),
\end{aligned}
$$

where $\lambda$ and $s^{\alpha}$ are as in $(7.5),(7.6)$ and $R_{\beta}^{\alpha}(\stackrel{\circ}{g}) \in S O(3)$ is given by the adjoint action of $\stackrel{\circ}{g}$ where we identify $s u(2)$ with the $\mathbb{R}^{3}$ of the Cartesian chart. In other words, it is the matrix satisfying,

$$
\stackrel{\circ}{g}\left(x^{\alpha} \tau_{\alpha}\right) \stackrel{\circ}{g}^{-1}=R_{\beta}^{\alpha}(\stackrel{\circ}{g}) x^{\beta} \tau_{\alpha} .
$$

Condition 7.13 can be seen to be equivalent to 7.10 by noting that $\stackrel{\circ}{E}^{\alpha}=\tau_{\alpha}$ and $\left(R^{-1}\right)_{\beta}^{\alpha}=$ $R_{\alpha}^{\beta}$

It follows from Appendix $\mathrm{C}$ that the set $\mathrm{Aut}^{\stackrel{\circ}{E}}$ of all such transformations preserve the asymptotic conditions (2.1) and $(2.2)$ and that $\mathrm{Aut}^{\stackrel{\circ}{E}}$ is a group. From 7.2 it follows that the group composition rule on Aut has the same 'semi-direct product' form (7.7) as in the Aut case. For later purposes we display the asymptotic form of a composed element $\left(g^{\prime \prime}, \phi^{\prime \prime}\right):=(g, \phi)\left(g^{\prime}, \phi^{\prime}\right)=\left(g \phi_{*} g^{\prime}, \phi \phi^{\prime}\right)$ (see appendix C 4):

$$
\begin{aligned}
g^{\prime \prime} & =\stackrel{\circ}{g} \stackrel{\circ}{ }^{\prime}+(\text { even }) r^{-1}+O\left(r^{-1-\epsilon}\right) \\
\phi^{\prime \prime \alpha} & =R_{\beta}^{\alpha}\left(\stackrel{\circ}{g} \stackrel{\circ}{ }^{\prime}\right) x^{\beta}+R_{\beta}^{\alpha}(\stackrel{\circ}{g}) t^{\prime \beta}+t^{\alpha}+(\text { odd })+O\left(r^{-\epsilon}\right) .
\end{aligned}
$$




\section{Aut $^{\stackrel{\circ}{E}} /$ Aut}

From the above discussion it is clear that Aut $\subset$ Aut $^{\stackrel{\circ}{ }}$. We now show it is actually a normal subgroup. Let $a \in$ Aut $^{\stackrel{\circ}{E}}$ and $b \in$ Aut. Since both groups are defined to be connected to identity, there exist paths $a(s) \in \mathrm{Aut}^{\stackrel{\circ}{E}}$ and $b(s) \in$ Aut with parameter $s \in[0,1]$ such that $a(0)=b(0)=\mathrm{Id}$ and $a(1)=a, b(1)=b$. Using (7.14), (7.15) it is straightforward to verify that

$$
c(s):=a(s) b(s) a(s)^{-1}
$$

satisfies the fall-offs associated to Aut. Since $c(0)=\mathrm{Id}, c(s)$ represents a path in Aut so that $c(1)=a b a^{-1} \in$ Aut. This shows normality of Aut $\subset$ Aut $^{E}$. We now describe the resulting quotient group $\mathrm{Aut}^{\stackrel{\circ}{E}} / \mathrm{Aut}$.

Elements of $\mathrm{Aut}^{\stackrel{\circ}{E}} /$ Aut are equivalence classes $[a], a \in \mathrm{Aut}^{\stackrel{\circ}{E}}$ where two elements $a, a^{\prime} \in$ Aut $^{\stackrel{\circ}{E}}$ are equivalent if $a a^{-1} \in$ Aut. Let us denote by

$$
\mathbf{t}: \mathrm{Aut}^{\stackrel{\circ}{E}} \rightarrow \mathbb{R}^{3}, \quad \mathbf{g}: \mathrm{Aut}^{\stackrel{\circ}{E}} \rightarrow S U(2),
$$

the maps that assign to $a \in \mathrm{Aut}^{\stackrel{\circ}{E}}$ the value of its asymptotic translation and $S U(2)$ rotation respectively. Thus for $a=(g, \phi)$ as in 7.11 and 7.12$), \mathbf{t}(a)=\vec{t}$ and $\mathbf{g}(a)=\stackrel{\circ}{g}$. We now show that

$$
[a]=\left[a^{\prime}\right] \Longleftrightarrow \mathbf{t}(a)=\mathbf{t}\left(a^{\prime}\right), \mathbf{g}(a)=\mathbf{g}\left(a^{\prime}\right),
$$

so that the space of equivalence classes may be identified with $\mathbb{R}^{3} \times S U(2)$. We will later describe the product rule between equivalence classes.

From Eqs. 7.14), 7.15) one has that for any $c \in$ Aut, $a \in \operatorname{Aut}^{\stackrel{\circ}{E}:} \mathbf{t}(a c)=\mathbf{t}(a)$ and $\mathbf{g}(a c)=\mathbf{g}(a)$. This establishes the $\Longrightarrow$ implication in $(7.18)$. To prove the converse we will construct a particular family of $\mathrm{Aut}^{\stackrel{\circ}{E}}$ elements with prescribed asymptotic values and show they exhaust all classes.

Any element $\stackrel{\circ}{g} \in S U(2)$ can always be written as

$$
\stackrel{\circ}{g}=e^{\theta \hat{n}},
$$

where $\hat{n} \equiv \hat{n}^{i} \tau_{i} \in S^{2} \subset s u(2)$ and $\theta \in[0,2 \pi]$ (values greater than $2 \pi$ can be reached by flipping $\left.\hat{n}, e^{(2 \pi+\alpha) \hat{n}}=e^{-(2 \pi-\alpha) \hat{n}}\right)$. This parametrization is one-to-one except at the 'poles' $\stackrel{\circ}{g}= \pm \mathbf{1}$, where $\theta=0,2 \pi$ and $\hat{n} \in S^{2}$ is undetermined. Given $(\hat{n}, \theta) \in S^{2} \times[0,2 \pi]$ and $\vec{t} \in \mathbb{R}^{3}$ define the diffeomorphisms:

$$
\begin{aligned}
\phi_{\vec{t}}^{\alpha}(x) & :=x^{\alpha}+t^{\alpha} f(r), \\
\phi_{(\hat{n}, \theta)}^{\alpha}(x) & :=R_{\beta}^{\alpha}\left(e^{\hat{n} f(r) \theta}\right) x^{\beta} .
\end{aligned}
$$

Here $f$ is a semianalytic $C^{k}$ interpolating function such that $f(r)=1$ for $r>r_{2}$ and $f(r)=0$ for $r<r_{1}$ (see Eq. (A7) of [13] for explicit example of such function). In addition we require that $f^{\prime}(r) \ll|\vec{t}|^{-1}$ so as to ensure invertibility of $\phi_{\vec{t}}^{\alpha} \cdot{ }^{16} R_{\beta}^{\alpha}\left(e^{\hat{n} f(r) \theta}\right)$ is the (r-dependent) $S O(3)$

16 Let $\tilde{\phi}_{\vec{t}}: \mathbb{R}^{3} \rightarrow \mathbb{R}^{3}$ be the map induced by $\phi_{\vec{t}}$ on $\mathbb{R}^{3}$ by setting $\left.\tilde{\phi}_{\vec{t}}\right|_{B_{r_{1}}}=$ Id where $B_{r_{1}}$ is the solid ball of radius $r_{1}$ in $\mathbb{R}^{3}$. Let $D_{\alpha}^{\beta}(x):=\partial_{\beta} \tilde{\phi}_{\vec{t}}^{\alpha}(x)=\delta_{\beta}^{\alpha}+t^{\alpha} \hat{x}_{\beta} f^{\prime}(r)$ be the differential of such map. Condition 
rotation associated to the ( $r$-dependent) $S U(2)$ element $e^{\hat{n} f(r) \theta}$ as in Eq. 7.13 . The inverse of (7.21) is given by $\phi_{(-\hat{n}, \theta)}$. By construction $\phi_{\vec{t}}$ and $\phi_{(\hat{n}, \theta)}$ are semianalytic diffeomorphisms that asymptote to a given translation and rotation respectively. Finally define ${ }^{17}$

$$
b_{(\vec{t}, \hat{n}, \theta)}:=\left(e^{\theta \hat{n}}, \phi_{\vec{t}} \circ \phi_{(\hat{n}, \theta)}\right) \in \operatorname{Aut}^{\stackrel{\circ}{E}} .
$$

By construction we have $\mathbf{t}\left(b_{(\vec{t}, \hat{n}, \theta)}\right)=\vec{t}$ and $\mathbf{g}\left(b_{(\vec{t}, \hat{n}, \theta)}\right)=e^{\theta \hat{n}}$. We now take care of a subtlety due to the $S U(2)$ parametrization being used. At $\theta=0, b_{(\vec{t}, \hat{n}, 0)}=\left(\mathbf{1}, \phi_{\vec{t}}\right)$ is independent of $\hat{n}$ and no issue arises. At $\theta=2 \pi, b_{(\vec{t}, \hat{n}, 2 \pi)}=\left(-\mathbf{1}, \phi_{\vec{t}} \circ \phi_{(\hat{n}, 2 \pi)}\right)$ depends on $\hat{n}$. We thus need to show that all such elements are in the same class, that is:

$$
b_{\left(\vec{t}, \hat{n}^{\prime}, 2 \pi\right)}^{-1} b_{(\vec{t}, \hat{n}, 2 \pi)}=\left(\mathbf{1}, \phi_{\left(\hat{n}^{\prime}, 2 \pi\right)}^{-1} \circ \phi_{(\hat{n}, 2 \pi)}\right) \in \text { Aut, } \quad \forall \hat{n}, \hat{n}^{\prime} \in S^{2} .
$$

To show (7.23) we need to find a one parameter family of diffeomorphisms $\phi_{s} \in$ Aut such that $\phi_{0}=\operatorname{Id}$ and $\phi_{1}=\phi_{\left(\hat{n}^{\prime}, 2 \pi\right)}^{-1} \circ \phi_{(\hat{n}, 2 \pi)}$. Consider a path $\hat{n}(s) \in S^{2}$ such that $\hat{n}(0)=\hat{n}$ and $\hat{n}(1)=\hat{n}^{\prime}$. Then

$$
\phi_{s}:=\phi_{(\hat{n}(s), 2 \pi)}^{-1} \circ \phi_{(\hat{n}, 2 \pi)}
$$

provides such path.

We finally show that any element in $\mathrm{Aut}^{\stackrel{\circ}{E}}$ is equivalent to one of the elements 7.22$)$. Let $a \in \mathrm{Aut}^{\stackrel{\circ}{E}}$ with asymptotic values $\vec{t}$ and $\stackrel{\circ}{g}=e^{\theta \hat{n}}$. We want to show that

$$
c:=b_{(\vec{t}, \hat{n}, \theta)} a^{-1}
$$

is in Aut. By construction $c$ has Aut-type fall-offs, so that all we need to show is that it is connected to identity. Let $a(s) \in$ Aut $^{\stackrel{\Xi}{E}}$ be a path connecting identity at $s=0$ to $a$ at $s=1$. Set $\vec{t}(s):=\mathbf{t}(a(s))$ and $\stackrel{\circ}{g}(s):=\mathbf{g}(a(s))$. For reasons that will become clear in a moment, we choose a parametrization $s \mapsto a(s)$ such that each time $\stackrel{\circ}{g}(s)$ goes through $\mathbf{- 1}$, it stays there for some closed interval $s \in\left[s_{i_{1}}, s_{i_{2}}\right] \subset[0,1]$ (this can always be achieved by an appropriate reparametrization $\left.s \rightarrow s^{\prime}(s)\right)$. A path $(\hat{n}(s), \theta(s))$ that is continuous in $S^{2} \times[0,2 \pi]$ and satisfies $e^{\theta(s) \hat{n}(s)}=\stackrel{\circ}{g}(s)$ can then be constructed as follows: For $\stackrel{\circ}{g}(s) \neq-\mathbf{1}$, the values of $(\hat{n}(s), \theta(s))$ are uniquely defined and the path is continuous. When $\stackrel{g}{g}(s)$ goes through $\mathbf{- 1}$, $\hat{n}$ may undergo a 'flip' $\hat{n} \rightarrow-\hat{n}$. For $s \in\left[s_{i_{1}}, s_{i_{2}}\right]$ where $\stackrel{\circ}{g}(s)=\mathbf{- 1}$, we specify $\hat{n}(s)$ so as to implement this flip in a continuous fashion i.e. we hold $\theta$ fixed at $2 \pi$ and continuously deform $\hat{n}$ to $-\hat{n}$. The corresponding path $b_{(\vec{t}(s), \hat{n}(s), \theta(s))}$ is thus continuous in Aut $^{\stackrel{\circ}{E}}$ (in the sense of footnote 15) and

$$
c(s):=b_{(\vec{t}(s), \hat{n}(s), \theta(s))} a^{-1}(s),
$$

provides the continuous path in Aut connecting identity with $c$ (7.25).

The above discussion establishes the one to one correspondence (7.18) which allow us to parametrize elements in the quotient space by pairs $(\vec{t}, \stackrel{\circ}{g}) \in \mathbb{R}^{3} \times S U(2)$. We now discuss

$f^{\prime}(r) \ll|\vec{t}|^{-1}$ implies that $\left(D^{-1}\right)_{\beta}^{\alpha}(x)$ exists and its coefficients are bounded as functions on $\mathbb{R}^{3}$. A version of Hadamard theorem then tells $\tilde{\phi}_{\vec{t}}$ is invertible in $\mathbb{R}^{3}$ (see theorem 6.2.3 of [30]). It then follows that $\left.\tilde{\phi}_{\vec{t}}^{-1}\right|_{B_{r_{1}}}=$ Id so that it can be extended to $\Sigma$ to define an inverse for $\phi_{\vec{t}}$.

17 To see that 7.22 is connected to identity consider the path $b(s):=\left(e^{s \theta \hat{n}}, \phi_{s \vec{t}} \circ \phi_{(\hat{n}, s \theta)}\right)$. Then $b(s)=\operatorname{Id}$ and $b(1)=b_{(\vec{t}, \hat{n}, \theta)}$. 
the product rule.

Recall the product on the quotient space is defined by $[a]\left[a^{\prime}\right]:=\left[a a^{\prime}\right]$. Given $a, a^{\prime} \in$ Aut $^{\stackrel{\circ}{E}}$ with asymptotic values $\vec{t}, \stackrel{\circ}{g}$ and $\vec{t}^{\prime}, \stackrel{\circ}{g}^{\prime}$, the asymptotic values of the product $a^{\prime \prime}:=a a^{\prime}$ can be read-off from (7.14), 7.15) (see appendix C4). From this we conclude the product rule on the quotient space is:

$$
(\vec{t}, \stackrel{\circ}{g})\left(\overrightarrow{t^{\prime}}, \stackrel{\circ}{g}^{\prime}\right)=\left(R(\stackrel{\circ}{g}) \overrightarrow{t^{\prime}}+\vec{t}, \stackrel{\circ}{g} \stackrel{\circ}{ }^{\prime}\right),
$$

which corresponds to the semidirect product of translations with $S U(2)$ rotations, $\mathrm{Aut}^{\stackrel{\circ}{E}} / \mathrm{Aut}=\mathbb{R}^{3} \rtimes S U(2)$.

We conclude the section with some comments. An analogous analysis in metric variables (where the relevant groups are the diffeomorphism parts of Aut, Aut ${ }^{\circ}$ ) shows that there can be two possible quotient groups: The standard isometry group of Euclidean space $\mathbb{R}^{3} \rtimes S O(3)$, or its cover $\mathbb{R}^{3} \rtimes S U(2)$. Which one arises depends on the topology of the manifold [14. For simple topologies, for instance $\Sigma=\mathbb{R}^{3}$, the quotient group has only the $S O(3)$ factor. In [14] it is described how certain topologies, associated with an $S U(2)$ factor in the quotient group, support odd-spin states in quantum theory. We will recover this result when studying asymptotic symmetries in quantum theory. In particular we will find that there are no 'new' odd-spin states arising from the use of triad rather than metric variables. Eventhough in the triad formulation the quotient group has always an $S U(2)$ rather than $S O(3)$ factor, the condition for existence of nontrivial odd-spin sates is still the same as the one described in [14] for metric variables.

\section{Action of Aut, Aut ${ }^{\stackrel{\circ}{E}}$ on elementary phase space functions}

Since gauge transformations are a subset of asymptotic symmetry transformations, the action of Aut on elementary phase space functions can be obtained by suitable restriction of that of $\mathrm{Aut}^{\stackrel{\circ}{E}}$ on these functions. Accordingly, we focus on the action of the larger group Aut $^{E}$. Given $(g, \phi) \equiv a \in$ Aut $^{\stackrel{\circ}{E}}$, we define its action on phase space functions $F[A, E]$ by: $(a \cdot F)[A, E]:=F\left[a^{-1} \cdot A, a^{-1} \cdot E\right]$ so that $(a b) \cdot F=a \cdot(b \cdot F)$. The elementary phase space functions (2.6), (2.8), 2.7) then transform as follows:

$$
\begin{aligned}
a \cdot h_{e}(A) & =g^{-1}(\phi(f(e))) h_{\phi(e)}(A) g(\phi(b(e))), \\
a \cdot F_{S, f}(E) & =F_{\phi(S), g \phi_{*} f g^{-1}}(E), \\
a \cdot \beta_{\bar{E}}[A] & =e^{i \alpha(a, \bar{E})} \beta_{a \cdot \bar{E}}[A],
\end{aligned}
$$

where $b(e), f(e)$ denote the beginning and end points of the edge $e$ and

$$
\alpha(a, \bar{E}):=\int_{\Sigma} \operatorname{Tr}\left[\phi_{*}\left(\bar{E}^{a}\right) g^{-1} \partial_{a} g\right]=\int_{\Sigma} \operatorname{Tr}\left[\left(a \cdot \bar{E}^{a}\right) \partial_{a} g g^{-1}\right] .
$$

Note that the set of phase space functions above is not preserved by action of arbitrary elements of $\mathrm{Aut}^{\stackrel{\circ}{E}}$ because such elements do not, in general, preserve the semianalyticity property of the probes. In order that the semianalyticity is preserved we further restrict attention to those elements of $\mathrm{Aut}^{\stackrel{\circ}{E}}$ which are semianalytic. It is then straightforward to check that such elements form a group. Let us call this group Aut $^{\stackrel{\circ}{E}}$ (sa).

Next, we show that the transformation laws 7.28)-7.30 hold. Since edges and surfaces 
are of compact support, Eqns. (7.28) and (7.29) follow by the same argument as in the case of compact $\Sigma[9$. To show (7.30) it will be convenient to study separately the actions $g \cdot \beta_{\bar{E}} \equiv(g, \mathrm{Id}) \cdot \beta_{\bar{E}}$ and $\phi \cdot \beta_{\bar{E}} \equiv(\mathrm{Id}, \phi) \cdot \beta_{\bar{E}}$, and later combine them by $(g, \phi)=(g, \mathrm{Id}) \circ(\mathrm{Id}, \phi)$. Even though asymptotic rotations do not admit such 'splitting' in phase space, one can make sense of such splitting in the space of connections $\mathcal{A}$ and of 'barred' electric fields $\mathcal{E}$.

First we notice that if $\rho$ is a scalar density such that $I:=\int_{\Sigma} \rho<\infty$ and $\phi$ a diffeomorphism of $\Sigma$ then $I=\int_{\Sigma} \phi_{*} \rho$. In particular for $\rho=\operatorname{Tr}\left[\bar{E}^{a} \phi_{*}^{-1} A_{a}\right]$ and $\phi \in \operatorname{Diff}_{\infty}$ this implies

$$
\phi \cdot \beta_{\bar{E}}=\beta_{\phi \cdot \bar{E}} .
$$

On the other hand, $g \cdot \beta_{\bar{E}}$ is given by the exponential of:

$$
\int_{\Sigma} \operatorname{Tr}\left[\bar{E}^{a}\left(g^{-1} A_{a} g+g^{-1} \partial_{a} g\right)\right]=\int_{\Sigma} \operatorname{Tr}\left[g \bar{E}^{a} g^{-1} A_{a}\right]+\int_{\Sigma} \operatorname{Tr}\left[\bar{E}^{a} g^{-1} \partial_{a} g\right] .
$$

Both integrals on the RHS are convergent since $g=\stackrel{\circ}{g}+($ even $) r^{-1}+O\left(r^{-1-\epsilon}\right)$ and $\partial_{a} g=$ (odd) $r^{-2}+O\left(r^{-2-\epsilon}\right)$. We thus conclude that $g \cdot \beta_{\bar{E}}=e^{i \alpha(g, \bar{E})} \beta_{g \cdot \bar{E}}$. Applying this result to $g \cdot \beta_{\phi \cdot \bar{E}}$ and using (7.32) we obtain (7.30) with $a=(g, \phi)$.

By construction, (7.28), 7.29) and 7.30 provide a representation of $\operatorname{Aut}^{\stackrel{\circ}{E}}$ (sa) on holonomies, fluxes and background exponentials.

\section{GAUGE TRANSFORMATIONS AND ASYMPTOTIC SYMMETRIES: QUANTUM IMPLEMENTATION.}

As in compact space LQG, we restrict attention to convenient subgroups of the automorphism groups Aut ${ }^{E}$, Aut. The restricted subgroups are chosen so as to incorporate the property of semianalyticity in such a way that the ensuing quantum theory is tractable, elegant and structurally rich. ${ }^{18}$ As seen in the previous section, the set of holonomy-background exponential- flux functions is invariant under the action of the subgroup $\mathrm{Aut}^{\stackrel{\dot{E}}{ }}$ (sa) of semianalytic elements of $\mathrm{Aut}^{\mathrm{E}}$. Here we further restrict the set of these transformations by a requirement of connection to identity. More in detail, we define the quantum symmetry group $A u t_{\text {sa }}^{\stackrel{E}{E}}$ to be the set of elements of $\operatorname{Aut}^{\stackrel{\Xi}{E}}$ (sa) which are connected to identity by paths

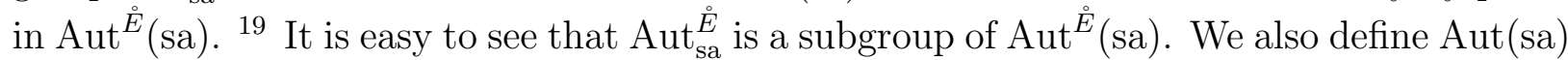
to be the set of semianalytic elements of Aut. It is easy to check that Aut(sa) is a subgroup of $\mathrm{Aut}^{\stackrel{\mathscr{E}}{(}}$ (sa). Finally we define the quantum gauge group Aut ${ }_{\mathrm{sa}}$ to be the set of elements of Aut(sa) which are connected to identity by paths in Aut(sa). It is straightforward to check that $\mathrm{Aut}_{\mathrm{sa}}$ is a subgroup of Aut(sa). The groups Aut $\mathrm{sa}_{\mathrm{sa}}^{\stackrel{\bullet}{E}}$, Aut $\mathrm{sa}_{\mathrm{sa}}$ are to be thought of as the quantum counterparts of the classical groups Aut ${ }^{\stackrel{\bullet}{E}}$, Aut. Clearly we have that Aut $_{\mathrm{sa}}$ is a subgroup of $\mathrm{Aut}_{\mathrm{sa}}^{E}$. It is then straightforward to see that the arguments of section VIIC apply unchanged if we replace $A u t^{\stackrel{E}{E}}$, Aut by $A u t_{\text {sa }}^{\stackrel{\leftrightarrow}{E}}$, Aut sa. It follows that Aut sa is a normal

\footnotetext{
${ }^{18}$ See section IX C for further discussion on the role of semianalyticity.

19 Note that any element in $\mathrm{Aut}^{\stackrel{\Xi}{E}}$ (sa) is connected to identity by paths in Aut ${ }^{\stackrel{\Xi}{ }}$. However it is not clear if any of these paths lie completely in $\operatorname{Aut}^{\stackrel{\complement}{E}}$ (sa). This is why we explicitly define Aut $t_{\text {sa }}^{\stackrel{\Xi}{E}}$. Similar comments apply to the necessity of defining Aut sa.
} 
subgroup of $\mathrm{Aut}_{\mathrm{sa}}^{\stackrel{\odot}{E}}$ and that the quotient group $\mathrm{Aut}_{\mathrm{sa}}^{\stackrel{\circ}{E}} / \mathrm{Aut}_{\mathrm{sa}}$ is, once again, $\mathbb{R}^{3} \rtimes S U(2)$.

In section VIII A we present a unitary action of Aut $\mathrm{sa}_{\mathrm{E}}^{\stackrel{\leftrightarrow}{E}}$ on $\mathcal{H}_{\mathrm{KS}}$. This unitary action,

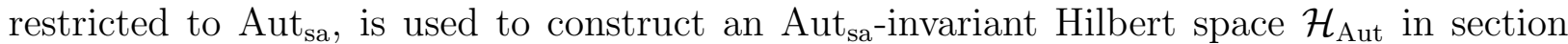
VIIIB. Section VIIIC discusses the unitary action of asymptotic rotations and translations on $\mathcal{H}_{\text {Aut }}$. Supplementary material to this section is given in appendix E.

\section{A. Unitary representation of $\mathrm{Aut}_{\mathrm{sa}}^{\stackrel{\circ}{E}}$ on the KS Hilbert space}

We wish to construct unitary operators $U(a): \mathcal{H}_{\mathrm{KS}} \rightarrow \mathcal{H}_{\mathrm{KS}}, a \in$ Aut $_{\mathrm{sa}}^{\stackrel{\bullet}{E}}$ satisfying $U(a b)=U(a) U(b), a, b \in \mathrm{Aut}_{\mathrm{sa}}^{\stackrel{\Xi}{E}}$ and such that they implement the analogue of the transformations $(7.28),(7.29),(7.30)$ for the corresponding operators $(3.2),(3.3),(3.5)$. The natural candidate is:

$$
U(a)|s, E\rangle:=e^{i \alpha(a, E)}\left|U^{\mathrm{LQG}}(a) s, a \cdot E\right\rangle,
$$

with $U^{\mathrm{LQG}}(a) s$ the usual action of $\mathrm{Aut}_{\mathrm{sa}}$ on spin networks and $a \cdot E$ as in $(7.2)$. In the case of compact $\Sigma$ the phase $\alpha(a, E)$ in $(8.1)$ is given by (7.31). This strategy does not work in the present case since the integral (7.31) is not guaranteed to be convergent if we replace $\bar{E}^{a}$ by $E^{a}$. Fortunately the divergent term is a total derivative that can be removed without affecting the composition property of the phases (Eq. (8.8) below) required for the satisfaction of $U(a b)=U(a) U(b)$. Given $a=(g, \phi)$, let

$$
\stackrel{\circ}{g}:=\lim _{r \rightarrow \infty} g
$$

be the zeroth order term of $g$. Thus $\stackrel{\circ}{g}=\mathbf{1}$ for $a \in$ Aut $_{\mathrm{sa}}$ and for asymptotic translations, whereas for asymptotic rotations $\stackrel{\circ}{g}$ is an internal rotation that ensures $E$ is fixed under the action of $a$. We define the phase $\alpha(a, E)$ in (8.1) by

$$
\alpha(a, E):=\int_{\Sigma} \rho(a, E)
$$

with

$$
\rho(a, E):=\operatorname{Tr}\left[a \cdot E^{a} \partial_{a} g g^{-1}\right]-\partial_{a} \operatorname{Tr}\left[E^{a} g g^{-1}\right] .
$$

In appendix E1 we show that the integral $(8.3)$ is finite. We now verify $U(a)$ is a representation of $\mathrm{Aut}_{\mathrm{sa}}^{\bar{E}}$ :

$$
U(a) U\left(a^{\prime}\right)=U\left(a a^{\prime}\right) \forall a, a^{\prime} \in \mathrm{Aut}_{\mathrm{sa}}^{\stackrel{\circ}{E}} .
$$

The action on a KS spinnet $|s, E\rangle$ of the operators on each side of (8.5) is:

$$
\begin{aligned}
U(a) U\left(a^{\prime}\right)|s, E\rangle & =e^{i \alpha\left(a, a^{\prime} \cdot E\right)} e^{i \alpha\left(a^{\prime}, E\right)}\left|U^{\mathrm{LQG}}\left(a a^{\prime}\right) s, a a^{\prime} \cdot E\right\rangle \\
U\left(a a^{\prime}\right)|s, E\rangle & =e^{i \alpha\left(a a^{\prime}, E\right)}\left|U^{\mathrm{LQG}}\left(a a^{\prime}\right) s, a a^{\prime} \cdot E\right\rangle,
\end{aligned}
$$

where we used that $U^{\mathrm{LQG}}\left(a a^{\prime}\right)=U^{\mathrm{LQG}}(a) U^{\mathrm{LQG}}\left(a^{\prime}\right)$ and $a \cdot\left(a^{\prime} \cdot E\right)=\left(a a^{\prime}\right) \cdot E$. In appendix E2 we show that

$$
\alpha\left(a a^{\prime}, E\right)=\alpha\left(a, a^{\prime} \cdot E\right)+\alpha\left(a^{\prime}, E\right)
$$

from which 8.5 follows.

It is straightforward to check that $U(a)$ preserves the inner product between KS spinnets. 
Finally, one can verify that $U(a)$ implements the transformations 7.28 , 7.29, 7.30 for the operators (3.2), (3.3), (3.5):

$$
\begin{aligned}
U(a) \hat{h}_{e} U(a)^{\dagger} & =g^{-1}(\phi(f(e))) \hat{h}_{\phi(e)} g(\phi(b(e))) \\
U(a) \hat{F}_{S, f} U(a)^{\dagger} & =\hat{F}_{\phi(S), g \phi_{*} f g^{-1}} \\
U(a) \hat{\beta}_{\bar{E}} U(a)^{\dagger} & =e^{i \alpha(a \bar{E})} \hat{\beta}_{a \cdot \bar{E}}
\end{aligned}
$$

where $a=(g, \phi)$. Again, because of the compact support property of $e$ and $S, \sqrt{8.9}$ and (8.10) follow from the same arguments given in the case of compact $\Sigma$ [9]. Eq. (8.11) can be shown by the same steps given in Eq. (C5) of [9] thanks to (8.8) and the fact that (8.3) satisfies (see appendix E1):

$$
\alpha(a, E+\bar{E})=\alpha(a, E)+\alpha(a, \bar{E})
$$

with $\alpha(a, \bar{E})$ as in 7.31 .

\section{B. Group averaging and Aut sa $_{\text {sanant }}$ Hilbert space $\mathcal{H}_{\text {Aut }}$}

\section{Setup}

In this section we denote KS spinnets labels as $\psi=(s, E)$, and the action of $\mathrm{Aut}_{\mathrm{sa}}$, Aut $\mathrm{sa}_{\mathrm{sa}}^{\stackrel{\mathrm{E}}{\mathrm{e}}}$ on these labels as $a \cdot \psi$. Let $\mathcal{D}_{\mathrm{KS}}$ be the dense subspace of $\mathcal{H}_{\mathrm{KS}}$ given by the finite linear span of KS spinnets. Let

$$
\mathrm{Ph}_{\psi}:=\left\{a \in \operatorname{Aut}_{\mathrm{sa}}: U(a)|\psi\rangle \propto|\psi\rangle\right\}, \quad \operatorname{Sym}_{\psi}:=\left\{a \in \operatorname{Aut}_{\mathrm{sa}}: U(a)|\psi\rangle=|\psi\rangle\right\} \subset \mathrm{Ph}_{\psi} .
$$

We start with the candidate for a group averaging map as in [9]:

$$
\eta(|\psi\rangle)=\left\{\begin{array}{cl}
0 & \text { if } \mathrm{Sym}_{\psi} \subsetneq \mathrm{Ph}_{\psi} \\
\eta_{[\psi]} \sum_{\mathbf{c} \in \mathrm{Autsa}_{\mathrm{sa}} / \mathrm{Sym}_{\psi}}\left(U\left(a_{\mathbf{c}}\right)|\psi\rangle\right)^{\dagger} & \text { if } \mathrm{Sym}_{\psi}=\mathrm{Ph}_{\psi},
\end{array}\right.
$$

where the sum is over the set of right cosets of $\mathrm{Aut}_{\mathrm{sa}}$ by $\mathrm{Sym}_{\psi}$, that is, the set of distinct $\operatorname{Sym}_{\psi}$-orbits $a \operatorname{Sym}_{\psi} \subset$ Aut $_{\text {sa }}$ for all $a \in$ Aut $_{\text {sa }} . a_{\mathbf{c}}$ is a choice of representative on each orbit c and $\eta_{[\psi]}$ are as yet unspecified positive constants that depend only on the Aut sa-orbit $[\psi]$ of $\psi$, that is $\eta_{[a \cdot \psi]}=\eta_{[\psi]} \forall a \in$ Aut $_{\mathrm{sa}}$. From the same arguments as in [9], (8.14) gives a well defined antilinear map $\eta: \mathcal{D}_{\mathrm{KS}} \rightarrow \mathcal{D}_{\mathrm{KS}}{ }^{\prime}\left(\mathcal{D}_{\mathrm{KS}}\right.$ ' the algebraic dual of $\left.\mathcal{D}_{\mathrm{KS}}\right)$, satisfying

$$
\begin{gathered}
\eta(U(a)|\psi\rangle)=\eta(|\psi\rangle) \forall a \in \text { Aut }_{\text {sa }}, \\
\eta\left(\left|\psi_{1}\right\rangle\right)\left[\left|\psi_{2}\right\rangle\right]=\frac{\eta\left(\left|\psi_{2}\right\rangle\right)\left[\left|\psi_{1}\right\rangle\right]}{\eta}, \quad \eta\left(\left|\psi_{1}\right\rangle\right)\left[\left|\psi_{1}\right\rangle\right] \geq 0 \quad \forall\left|\psi_{1}\right\rangle,\left|\psi_{2}\right\rangle \in \mathcal{D}_{\mathrm{KS}} .
\end{gathered}
$$

The last property allows one to define the inner product $\left.\left\langle\eta\left(\left|\psi_{1}\right\rangle\right)\right| \eta\left(\left|\psi_{2}\right\rangle\right)\right\rangle_{\eta}:=\eta\left(\left|\psi_{1}\right\rangle\right)\left[\left|\psi_{2}\right\rangle\right]$ that is used in the definition of the Aut $_{\text {sa }}$-invariant Hilbert space $\mathcal{H}_{\text {Aut }}[1,9]$.

There remains to be imposed a third requirement on $\eta$ that allows to define 'observables' in $\mathcal{H}_{\text {Aut }}$ from operators in $\mathcal{H}_{\mathrm{KS}}$. Let $\mathcal{O}$ be the set of operators on $\mathcal{H}_{\mathrm{KS}}$ such that $O \in \mathcal{O}$ 
satisfies (i) both $O$ and $O^{\dagger}$ are defined on $\mathcal{D}_{\mathrm{KS}}$ and their action preserves $\mathcal{D}_{\mathrm{KS}}$; (ii) $U(a) O=$ $O U(a) \forall a \in$ Aut. Following [1] we require:

$$
\eta\left(\left|\psi_{1}\right\rangle\right)\left[O\left|\psi_{2}\right\rangle\right]=\eta\left(O^{\dagger}\left|\psi_{1}\right\rangle\right)\left[\left|\psi_{2}\right\rangle\right] \quad \forall \psi_{1}, \psi_{2} \in \mathcal{D}_{\mathrm{KS}}, \forall O \in \mathcal{O} .
$$

This condition ensures that all such $O$ obey, as operators in $\mathcal{H}_{\text {Aut }}$, the same adjointness relations as operators in $\mathcal{H}_{\mathrm{KS}}$. In particular, if $O$ is unitary as operator in $\mathcal{H}_{\mathrm{KS}}$, it guarantees $O$ is unitary as an operator in $\mathcal{H}_{\text {Aut }}$. As in [9], we shall see that (8.17) determines the coefficients $\eta_{[\psi]}$ within a 'superselection sector' (see below).

It is however not obvious that condition (8.17) will guarantee a unitary action of asymptotic rotations and translations, $U(b), b \in \mathrm{Aut}_{\mathrm{sa}}^{E}$, since these operators do not belong to $\mathcal{O}$ for the following reason: whereas $\mathcal{O}$ defined above consists of operators that commute 'strongly' with the gauge group, asymptotic rotations and translations commute 'weakly' with the gauge group. That is, instead of satisfying (ii) in the definition of $\mathcal{O}$, they satisfy: (ii)': Given $a \in \mathrm{Aut}_{\mathrm{sa}}, O U(a)=U\left(a^{\prime}\right) O$ for some $a^{\prime} \in \mathrm{Aut}_{\mathrm{sa}}$. Whereas (i) and (ii)' are sufficient conditions to be able to define $O$ as an operator on $\mathcal{H}_{\text {Aut }}$, it is a priori not clear that such operator will satisfy the correct adjointess properties. In subsection VIIIC we will verify that rotations and translation do act unitarily in $\mathcal{H}_{\text {Aut }}$.

\section{Superselection sectors}

By 'superselection sector' we will mean a subspace of $\mathcal{H}_{\text {Aut }}$ such that any two elements in the subspace can be mapped into each other by an operator in $\mathcal{O}$ or by some $U(b), b \in \mathrm{Aut}_{\mathrm{sa}}{ }^{E}{ }^{20}$ If $\eta(|\psi\rangle)$ and $\eta\left(\left|\psi^{\prime}\right\rangle\right)$ lie in the same (different) superselection sector, we shall say that $|\psi\rangle$ and $\left|\psi^{\prime}\right\rangle$ lie in the same (different) superselection sector.

Given an asymptotically flat $E^{a}$, we define its rank sets as in [9] by

$$
\begin{aligned}
& V_{0}^{E}:=\{x \in \Sigma: \operatorname{rank}(E)=0\}, \\
& V_{1}^{E}:=\{x \in \Sigma: \operatorname{rank}(E)=1\}, \\
& V_{2}^{E}:=\{x \in \Sigma: \operatorname{rank}(E) \geq 2\} .
\end{aligned}
$$

In appendix E3 we show the rank sets can be decomposed into finite union of semianalytic submanifolds. Diff-classes of these sets can be used as partial labels for superselection sectors as follows.

By the same arguments given in [9], it follows that

$$
\begin{array}{ll}
\left\langle s, E|O| s^{\prime}, E^{\prime}\right\rangle \neq 0 \Longrightarrow & \text { i) } \quad \stackrel{\circ}{V}_{0}^{E}=\stackrel{\circ}{V}_{0}^{E^{\prime}}, \quad \bar{V}_{2}^{E}=\bar{V}_{2}^{E^{\prime}} \\
& \text { ii) } \quad \operatorname{dim}\left(V_{n}^{E} \cap\left(\cup_{n^{\prime} \neq n} V_{n^{\prime}}^{E^{\prime}}\right)\right)<3, n=0,1,2 \\
& \text { iii) } \tilde{\gamma}(s) \cap \stackrel{\circ}{V}_{0}^{E}=\tilde{\gamma}\left(s^{\prime}\right) \cap \stackrel{V}{0}_{0}^{E},
\end{array}
$$

where $\tilde{\gamma}(s), \tilde{\gamma}\left(s^{\prime}\right)$ are the graphs of the spin networks, and dim denotes the dimension of

20 The inclusion of $U(b), b \in \mathrm{Aut}_{\mathrm{sa}}^{\stackrel{̊}{E}}$ in this definition may be redundant. It may be the case that any superselection sector defined only with respect to $\mathcal{O}$ is automatically invariant under the action of $U(b), b \in$ $\mathrm{Aut}_{\mathrm{sa}}^{\stackrel{\leftrightarrow}{E}}$. 
the sets in (ii) (which are also composed of finite union of semianalytic submanifolds, see appendix E3). As in [9], these conditions imply that for any pair of KS spinnets $|s, E\rangle$ and $\left|E^{\prime}, s^{\prime}\right\rangle$ lying in a same superselection sector there exists a diffeomorphism $\phi$ such that 8.21 , 8.22 and 8.23 hold with $\phi\left(V_{n}^{E^{\prime}}\right)$ in place of $V_{n}^{E^{\prime}}$ and $\phi\left(s^{\prime}\right)$ in place of $s^{\prime}$.

\section{Group averaging in the absence of rank 1 backgrounds}

We now focus on a particular class of superselection sectors, and discuss how the coefficients $\eta_{[\psi]}$ are determined. The type of states we consider are the analogue of those described in section 7 of [9]: $|\psi\rangle=|s, E\rangle$ such that: (1) $V_{1}^{E}$ is of zero measure, (2) the only infinitesi-

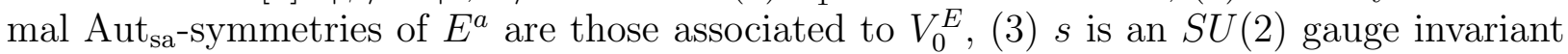
spin network, (4) $\mathrm{Ph}_{\psi}=\mathrm{Sym}_{\psi}$. Conditions (1) to (4) give a set of consistent restrictions in the sense described in $[9] .{ }^{21}$ Next, we define

$$
\operatorname{Sym}_{\psi}^{0} \equiv \operatorname{Sym}_{(s, E)}^{0}:=\left\{a \in \operatorname{Aut}_{\mathrm{sa}}:\left.a\right|_{\bar{V}_{2}^{E}}=\mathrm{Id}, a(\tilde{e})=\tilde{e} \quad \forall \tilde{e} \in s\right\},
$$

where $\bar{V}_{2}^{E}$ is the closure of $V_{2}^{E}, \tilde{e} \in s$ denotes the edges in $s$ (as 1-dimensional manifolds) and $a(\tilde{e}) \equiv(g, \phi)(\tilde{e}):=\phi(\tilde{e})$. It is easy to verify that $\operatorname{Sym}_{\psi}^{0}$ is a normal subgroup of $\operatorname{Sym}_{\psi}$, and as in [9] we assume the corresponding quotient group

$$
D_{\psi}:=\operatorname{Sym}_{\psi} / \operatorname{Sym}_{\psi}^{0}
$$

is finite, this finiteness being expected from that of the group of allowed edge permutations of $\gamma(s)$ and of the discrete symmetries of the background. By the same arguments given in [9], condition (8.17) is then satisfied if and only if

$$
\eta_{[\psi]}=C\left|D_{\psi}\right|
$$

for some constant $C>0$. Thus, the ambiguity in the coefficients $\eta_{[\psi]}$ is reduced to one constant per each superselection sector.

\section{Asymptotic rotations and translations on $\mathcal{H}_{\text {Aut }}$}

\section{Unitary action}

We now discuss the analogue of condition (8.17) for asymptotic rotations and translations: ${ }^{22}$

$$
\eta\left(\left|\psi_{1}\right\rangle\right)\left[U(b)\left|\psi_{2}\right\rangle\right]=\eta\left(U^{\dagger}(b)\left|\psi_{1}\right\rangle\right)\left[\left|\psi_{2}\right\rangle\right] \quad \forall\left|\psi_{1}\right\rangle,\left|\psi_{2}\right\rangle \in \mathcal{D}_{\mathrm{KS}}, \forall b \in \mathrm{Aut}_{\mathrm{sa}}^{\stackrel{\bullet}{E}} .
$$

${ }^{21}$ Condition (3) should actually be improved upon to allow for gauge variant spin networks, see discussion of section $9 \mathrm{~B}$ in $[9]$.

${ }^{22}$ If 8.27) holds for some $b$, it automatically holds for $b^{\prime}=b a$ and $b^{\prime \prime}=a b, \forall a \in \mathrm{Aut}_{\mathrm{sa}}$, so that the set of independent conditions in 8.27 are parametrized by $\mathrm{Aut}_{\mathrm{sa}}^{\stackrel{\leftrightarrow}{E}} / \mathrm{Aut}_{\mathrm{sa}}$. Similarly, one has that $U(b), U(a b)$ and $U(b a)$, define the same operator on $\mathcal{H}_{\text {Aut }} \forall a \in$ Aut $_{\text {sa }}$. 
We first note that the coefficients $\eta_{[\psi]}$ determined in the last section by Eq. (8.26) satisfy

$$
\eta_{[b \cdot \psi]}=\eta_{[\psi]}, \forall b \in \operatorname{Aut}_{\mathrm{sa}}^{\stackrel{̊}{E}} .
$$

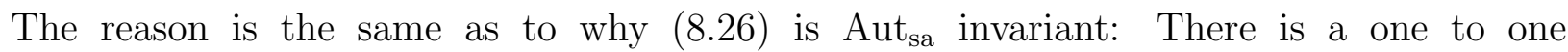
correspondence between elements of $\mathrm{Sym}_{\psi} / \mathrm{Sym}_{\psi}^{0}$ and elements of $\mathrm{Sym}_{b \cdot \psi} / \mathrm{Sym}_{b \cdot \psi}^{0}=$ $b \operatorname{Sym}_{\psi} b^{-1} / b \operatorname{Sym}_{\psi}^{0} b^{-1}$ given by: $[s]=s \operatorname{Sym}_{\psi}^{0} \in \operatorname{Sym}_{\psi} / \operatorname{Sym}_{\psi}^{0} \Longleftrightarrow b[s] b^{-1} \in \operatorname{Sym}_{b \cdot \psi} / \operatorname{Sym}_{b \cdot \psi}^{0}$, so that $\left|D_{\psi}\right|=\left|D_{b \cdot \psi}\right|$.

We now show that Eq. (8.28) implies Eq. (8.27). Consider the following equivalent form of Eq. (8.27):

$$
\eta(U(b)|\psi\rangle)(U(b)|\phi\rangle)=\eta(|\psi\rangle)(|\phi\rangle) \quad \forall|\psi\rangle,|\phi\rangle \in \mathcal{D}_{\mathrm{KS}}, \forall b \in \mathrm{Aut}_{\mathrm{sa}}^{\stackrel{\bullet}{E}},
$$

Using (8.14), the left hand side of $(8.29)$ can be written as:

$$
\eta(U(b)|\psi\rangle)(U(b)|\phi\rangle)=\eta_{[b \cdot \psi]} \sum_{\mathbf{c} \in \operatorname{Aut}_{\mathrm{sa}} / \mathrm{Sym}_{b \cdot \psi}}\left\langle\psi\left|U^{\dagger}\left(b^{-1} a_{\mathbf{c}} b\right)\right| \phi\right\rangle .
$$

Next, we note that $\operatorname{Sym}_{b \cdot \psi}=b \operatorname{Sym}_{\psi} b^{-1}$ and that there is a one to one correspondence between $\mathrm{Aut}_{\mathrm{sa}} / \mathrm{Sym}_{\psi}$ and $\mathrm{Aut}_{\mathrm{sa}} /\left(b \mathrm{Sym}_{\psi} b^{-1}\right)$ given by

$$
\mathbf{c} \in \mathrm{Aut}_{\mathrm{sa}} /\left(b \operatorname{Sym}_{\psi} b^{-1}\right) \Longleftrightarrow b^{-1} \mathbf{c} b \in \mathrm{Aut}_{\mathrm{sa}} / \mathrm{Sym}_{\psi} .
$$

Further, $b^{-1} a_{\mathbf{c}} b \in b^{-1} \mathbf{c} b$. Thus, the sum on the right hand side of 8.30 takes the same form as the sum in the definition of $\eta[|\psi\rangle](|\phi\rangle)$. Using (8.28), equation (8.29) follows.

Recall from section VIIC that $\mathrm{Aut}_{\mathrm{sa}}^{\mathrm{E}} / \mathrm{Aut}_{\mathrm{sa}}=\mathbb{R}^{3} \rtimes S U(2)$. Let $b_{\vec{t}}, b_{\grave{g}} \in \mathrm{Aut}_{\mathrm{sa}}^{\stackrel{\bullet}{E}}$ denote representatives of a translation $\vec{t} \in \mathbb{R}^{3}$ and a rotation $\stackrel{\circ}{g} \in S U(2)$. The unitary action of $\vec{t}, \vec{g}$ on $|\psi\rangle:=\eta(|\psi\rangle) \in \mathcal{H}_{\text {Aut }}$ can then be written as:

$$
\left.U(\vec{t}) \mid \psi):=\eta\left(U\left(b_{\vec{t}}\right)|\psi\rangle\right), \quad U(\stackrel{\circ}{g}) \mid \psi\right):=\eta\left(U\left(b_{\grave{g}}\right)|\psi\rangle\right) .
$$

\section{Friedman-Sorkin 'Spin 1/2 from Gravity' States}

In [14, Friedman and Sorkin (FS) provide a general argument for the appearance of oddspin states in a quantum theory of the gravitational field. The argument may be summarized as follows. Consider a theory of quantum gravity with states given by wavefunctions of the 3 metric on the spatial slice. The diffeomorphism constraint implies the physical wavefunctions are invariant under the action of diffeomorphisms that are trivial at infinity and connected to identity. Let us denote by 'gauge' such diffeomorphisms. Let $\phi_{\mathrm{FS}}$ be a diffeomorphism that interpolates between a $2 \pi$ rotation at infinity with identity in the interior (for instance take $\phi_{\mathrm{FS}}:=\phi_{\left(\tau_{3}, 2 \pi\right)}$ as in Eq. (7.21)). Doubly connectedness of $S O(3)$ implies that $\phi_{\mathrm{FS}} \circ \phi_{\mathrm{FS}}$ is guage (an argument for this follows from the discussion leading to Eq. (7.24)). Thus on the physical space:

$$
\hat{\phi}_{\mathrm{FS}} \hat{\phi}_{\mathrm{FS}}=\mathrm{Id} \text {. }
$$

We now ask if $\phi_{\mathrm{FS}}$ is gauge, i.e. if it can be continuosly deformed to identity in such a way that it stays trivial at infinity. It turns out that the answer depends on the topology of 
the manifold $\Sigma$. The characterization of all possible topologies for which $\phi_{\mathrm{FS}}$ is not gauge is available in the mathematics literature and described in [14. Manifolds $\Sigma$ with such topologies are termed 'spinorial'. For spinorial manifolds, there are states $\psi$ such that

$$
\psi^{\prime}:=\hat{\phi}_{\mathrm{FS}} \psi \neq \psi \text {. }
$$

It then follows that the state $\psi^{\prime}-\psi$ exhibits odd spin behaviour under the asymptotic $2 \pi$ rotation:

$$
\hat{\phi}_{\mathrm{FS}}\left(\psi^{\prime}-\psi\right)=-\left(\psi^{\prime}-\psi\right) \text {. }
$$

The adaptation of these ideas to our model are straightforward. We only need to keep track of additional $S U(2)$ rotations. ${ }^{23}$ In our language, odd spin states can arise if $\mathbf{- 1} \in$ $S U(2) \subset \mathrm{Aut}_{\mathrm{sa}}^{\stackrel{\varrho}{E}} /$ Aut $_{\mathrm{sa}}$ acts non-trivially in $\mathcal{H}_{\text {Aut }}$. Let

$$
b_{-1}:=\left(-1, \phi_{\mathrm{FS}}\right) \text {, }
$$

be a representative of the type given in section VIIC, If $\Sigma$ is not spinorial, we can deform $b_{-1}$ through Aut by deforming the diffeomorphisms while keeping the $\mathbf{- 1}$ factor fixed. In this way one then obtains a new representative $b_{-\mathbf{1}}^{\prime}:=(-\mathbf{1}$, Id). It is easy to see that $U\left(b_{-1}^{\prime}\right)|s, E\rangle=|s, E\rangle$ for all KS spinnets. This implies $U(-\mathbf{1})=$ Id on $\mathcal{H}_{\text {Aut }}$ so that no odd spin states arise.

For spinorial $\Sigma$ the argument above does not apply and odd spin states may be constructed as in the above FS argument, for instance by considering states of the form $\left|\left(\phi_{\mathrm{FS}}\right)_{*} \bar{E}\right\rangle-|\bar{E}\rangle$. Note that in this case $(-\mathbf{1}, \mathrm{Id}) \notin \mathrm{Aut}_{\mathrm{sa}}^{\stackrel{\mathscr{E}}{E}}$ as there is no path in $A u t_{\mathrm{sa}}^{\stackrel{\bullet}{E}}$ connecting this element to identity. In particular $\left(-\mathbf{1}\right.$, Id) is not a representative of $-\mathbf{1} \in S U(2) \subset \mathrm{Aut}_{\mathrm{sa}}^{\stackrel{\leftrightarrow}{E}} / \mathrm{Aut}_{\mathrm{sa}}$ as it was in the non-spinorial manifold case.

\section{Comments on eigenstates of linear and angular momentum}

We conclude the section with some comments regarding the possibility of finding states with definite linear or angular momentum. A first example is given by the case where $\Sigma=\mathbb{R}^{3}$ and $\left|\psi_{0}\right\rangle:=|0, \stackrel{\circ}{E}\rangle$ where 0 denotes the trivial spin network and $\stackrel{\circ}{E}^{a}$ the flat triad on $\mathbb{R}^{3}$. The corresponding Aut-invariant state $\eta\left(\left|\psi_{0}\right\rangle\right)$ is left invariant under $U(\stackrel{\circ}{g}), U(\vec{t}), \forall \stackrel{\circ}{\mathrm{g}} \in$ $S U(2), \vec{t} \in \mathbb{R}^{3}$ and hence $\eta\left(\left|\psi_{0}\right\rangle\right)$ is a state with zero linear and angular momenta. A similar state with $E^{a}$ replaced by a spherically symmetric triad (but not translation invariant) yields a state with zero angular moment and indefinite linear momentum. We have not succeeded in finding examples of normalizable states in $\mathcal{H}_{\text {Aut }}$ with definite nonzero momenta. On the other hand, while we do not do so here, distributional states with definite linear/angular momenta can be constructed by group averaging over the appropriately weighted action of asymptotic translations/rotations on suitable states in $\mathcal{H}_{\text {Aut }}$.

${ }^{23}$ Another difference which does not alter the argument is the presence of odd supertranslations which are non-trivial at infinity but still 'gauge'. 


\section{CONCLUSIONS}

\section{A. Summary of results}

In this work we developed a quantum kinematics for asymptotically flat gravity which suitably addresses the classical asymptotic conditions on the triad and connection variables of the theory. The quantum kinematics supports a unitary representation of the gauge group Aut $_{\text {sa }}$ (suitably restricted by the requirement of semianalyticity and connectedness to identity) of internal rotations which asymptote to identity and spatial diffeomorphisms which asymptote to odd supertranslations. The kinematics also supports a unitary representation of the larger group Aut $t_{\mathrm{sa}}^{\stackrel{\leftrightarrow}{E}}$ of asymptotic symmetries (also subject to semianalyticity and connectedness to identity restrictions) which, modulo gauge, asymptote to non-trivial rotations and translations at spatial infinity. Similar to the case of compact space LQG, solutions to the Gauss Law and spatial diffeomorphism constraints are identified with states which are invariant under the action of Aut $\mathrm{sa}$. We constructed a large sector of the Hilbert space of such gauge invariant states using group averaging methods. As in the case of compact space LQG, this sector is superselected with respect to the action of a certain set of gauge invariant observables. ${ }^{24}$ We showed that the group of asymptotic rotations and translations (obtained

as the quotient of the symmetry group Aut $t_{\mathrm{sa}}^{E}$ by the gauge group Aut $_{\mathrm{sa}}$ ) is implemented unitarily on this sector. Finally, following Friedman and Sorkin [14 we showed that for $\Sigma$ with appropriate topology, the gauge invariant Hilbert space contains states that change by a sign under a $2 \pi$ rotation.

\section{B. Remarks and caveats of a technical nature}

While, in sections III $[\mathrm{VI}$, we used asymptotic boundary conditions corresponding to finite differentiability versions of the so called 'parity' conditions [17-19], our considerations in these sections are expected to go through for any choice of asymptotic conditions which implement asymptotic flatness in such a way that the phase space is realized as a cotangent bundle over the configuration space of connections. The subsequent developments in sections VII and VIII do, however, depend on the detailed choice of these parity conditions. More in detail, the demonstration of the finiteness of the phase factors (7.31) and (8.3) as well as the composition property 8.8 depend crucially on the parity conditions and would have to be checked explicitly for any other choice of asymptotic behaviour.

Regarding the restriction to semianalytic fields: The KS background electric field labels have the double condition of satisfying (2.1) and being semianalytic. In appendix A we show there exist a rich family of functions satisfying both the required fall-offs and semianaliticity conditions. Similarly, the group elements of $A u t_{\mathrm{sa}}$ and $A u t_{\mathrm{sa}}^{\stackrel{\mathscr{E}}{2}}$ are required to be semianalytic in addition to possess the fall-offs described in sections VIIA and VIIB. Explicit examples of such (connected to identity) elements with nontrivial asymptotic translations and rotations appear in section VIIC. It is possible to construct by the same methods examples of Aut $\mathrm{t}_{\mathrm{sa}}$ elements with non-trivial asymptotic supertranslations.

${ }^{24}$ We do not expect that these 'kinematical' superselection sectors will be preserved by the Hamiltonian constraint. 
Our final remark is concerned with a technicality in our treatment of group averaging in section VIII B 3. Similar to Reference [9] we assumed finiteness of the group $D_{\psi}$ defined in Eq. 8.25 .

\section{The role of semianalytic structures}

Let us first discuss the role of semianalytic structures in compact space LQG. One may attempt to view compact space LQG (on a $C^{k}$ Cauchy slice admitting a semianalytic structure) as a quantization of a Hamiltonian formulation of gravity with $C^{k-1}$ triads and $C^{k-2}$ connections defined on this slice.

In this view, the classical holonomy-flux algebra may be obtained by smearing electric fields along semianalytic $C^{k}$ surfaces $S$ and semianalytic $C^{k-1}$ functions $f$ thereon; and by smearing the connections along semianalytic $C^{k}$ edges. This algebra is invariant under semianalytic $C^{k-1} S U(2)$ rotations and semianalytic $C^{k}$ diffeomorphisms. Therefore the kinematical gauge group of LQG is taken to be the semidirect product of semianalytic $S U(2)$ rotations with semianalytic diffeomorphisms. In the process, we see that the generic finite differentiability gauge transformations of the classical theory have been restricted to be semianalytic. Nevertheless, since the quantum theory is so elegantly formulated for the semianalytic category and since the quantum excitations which are invariant under both the kinematical gauge transformations as well as the (putative) action of the Hamiltonian constraint are expected to be very different from the kinematic ones, it is a good strategy to explore where this relatively mild restriction leads us to. Indeed, our view is that (i) this strategy is likely to be too conservative to construct the final quantum theory but (ii) that nevertheless pursuing this strategy will provide enough information to motivate more radical but educated departures therefrom so as to construct fundamental quantum spacetime structures. Indeed, in the final picture one would perhaps expect that the spacetime manifold only emerges at scales much larger than the Planck scale. Similar remarks apply to the KS representation for compact spaces. Therefore our view is that one should not worry too much about the exact class of differential structures one uses in quantum theory relative to classical theory.

At this point it is necessary to make the following caveat regarding classical Hamiltonian theory in the $C^{k}$ setting. The infinitesimal transformations generated by the diffeomorphism and Hamiltonian constraints involve single spatial derivatives of the fields so that the infinitesimal variations of the fields suffer a drop of degree of differentiability. Consequently, these transformations do not leave the phase space of finite differentiability fields invariant. While it may well be the case that the consideration of finite transformations remedies this defect for flows corresponding to a combination of arbitrary shifts and non-vanishing lapses ${ }^{25}$ we are not aware of a demonstration of this property. However, with our view on classical structures emerging from quantum ones at large distance scales, we are not unduly concerned if such a property holds; it is enough for us that the quantum theory is based on structures which are similar but not necessarily identical to the classical case. Since the problems discussed in this paragraph disappear for $C^{\infty}$ fields, in our view, it is an acceptable

${ }^{25}$ If the lapse vanishes, standard results 31 indicate that the diffeomorphism constraint smeared with a $C^{k-1}$ shift generates $C^{k-1}$ diffeomorphisms. Such diffeomorphisms in general map a $C^{k-1}$ tensor field to a $C^{k-2}$ one, so that phase space is not preserved. 
strategy to (a) restrict the $C^{\infty}$ category to the semianalytic one so as to construct an algebra which admits an elegant quantization for which a large class of diffeomorphisms (namely the semianalytic ones) act unitarily, (b) to define kinematically gauge invariant states as those invariant under suitable semianalytic transformations and (c) to then attempt to impose the Hamiltonian constraint on this space.

Relative to the compact case, in the asymptotically flat case we need to specify not only the differential structure of the fields considered but also their asymptotic behaviour. Once again for the reasons discussed above, the simplest consistent Hamiltonian formulations are defined in the $C^{\infty}$ setting. The fall offs we would like to use are those provided by the parity conditions of References [17, 19]. As indicated in section II B the subleading terms in these fall offs are only defineable in the $C^{\infty}$ setting. Nevertheless, guided by the role of semianalytic structures in quantum theory in the compact space setting, we would like to base our considerations on the $C^{k}$ semianalytic differential structure while still making contact with the parity conditions in some way. For the phase space variables, this leads to the conditions (2.1), (2.2) which are closely related to the parity conditions in that while the leading order terms look identical to those for the $C^{\infty}$ case, the fall offs of the subleading terms are restricted by their finite differentiability. Similarly, the asymptotic behaviour of the gauge and symmetry transformations (as described in sections VII A and VII B) is closely related to their $C^{\infty}$ counterparts in appendix $\mathrm{D}$.

\section{Future work}

Our considerations are based on the KS representation which is a generalization of standard compact space LQG [6, 7, 9, 12, 13, to account for non-trivial 'background' spatial geometries, the LQG representation being associated with a 'zero background spatial geometry' state corresponding to a vanishing (and hence, degenerate) triad field. Two distinct research directions suggest themselves depending on whether one views the KS representation as fundamental or whether one views the KS representation as an effective description of fundamental LQG excitations. If one views the KS representation as fundamental, the next step would be to develop an understanding of the Hamiltonian constraint operator in this representation. If one views the LQG representation as fundamental then it would be of great interest to use the KS kinematics developed here as a template for the development of asymptotically flat LQG, building on the earlier work of references [10, 11].

Irrespective of which line of thought one pursues, key physical issues can only be addressed once one has an adequate understanding of the Hamiltonian constraint (in the LQG context see [33-38]; in the KS context see [39, 40]). In our view, the two most important and crucial issues are the existence of (A) a quantum positive energy theorem 26 and (B) a unitary representation of asymptotic Poincare transformations (including time translations and boosts, both of which require an understanding of the Hamiltonian constraint operator). Indeed, any understanding of these issues, even without a full understanding of the Hamiltonian constraint itself, would be very exciting and would go a long way in showing the physical viability of the theory.

Acknowledgements: We are indebted to Fernando Barbero and Hanno Sahlmann for

\footnotetext{
${ }^{26}$ See Reference [32] for first steps, albeit in the context of self dual rather than real connection variables.
} 
their comments on a draft version of this work. MC thanks Alok Laddha for helpful discussions.

\section{Appendix A: Semianalyticity of functions on $\chi_{0}\left(U_{0}\right)$ from that of functions on $S^{2}$.}

$\chi_{0}\left(U_{0}\right)$ can be given an analytic structure compatible with the preferred Cartesian chart $x^{\alpha}$. In (1)- (3a) below we shall treat $\chi_{0}\left(U_{0}\right)$ as an analytic manifold with this analytic structure.

(1) Let $S^{2}$ denote the topological 2 sphere. We endow $S^{2}$ with an analytic atlas and denote the resulting analytic manifold by $S_{w}^{2}$ so that $S^{2}$ and $S_{w}^{2}$ are identical as point sets and topologies. Let $(u, v)_{N, S}$ be the (analytic) stereographic coordinate charts associated with $S^{2}-N, S^{2}-S$ where $N, S$ are the North and South Poles of $S^{2}$. Define the natural analytic map $g: \chi_{0}\left(U_{0}\right) \rightarrow S_{w}^{2}$ in the charts $x^{\alpha}$ and $(u, v)_{N, S}$ as:

$$
(u, v)_{N}=\left(\frac{x^{1}}{1-x^{3}}, \frac{x^{2}}{1-x^{3}}\right), \quad(u, v)_{S}=\left(\frac{x^{1}}{1+x^{3}},-\frac{x^{2}}{1+x^{3}}\right)
$$

This implies that given any analytic chart $x^{A}$ on $S_{w}^{2}$, we have that:

(i) $g^{A}\left(x^{\alpha}\right) \equiv x^{A}\left(x^{\alpha}\right)$ are analytic functions of $x^{\alpha}$.

(ii) $\left(r\left(x^{\alpha}\right)=\sqrt{x^{\alpha} x_{\alpha}}, x^{A}\left(x^{\alpha}\right)\right)$ are analytic coordinates on $\chi_{0}\left(U_{0}\right)$.

(2) Consider a maximal $C^{n}$ semianalytic atlas on $S^{2}$ which contains the analytic charts of (1). This atlas endows $S^{2}$ with the structure of a $C^{n}$ semianalytic manifold. We refer to it as $S_{n}^{2}$ so that as point sets and topologies $S^{2}=S_{w}^{2}=S_{n}^{2}$. It is easy to check that the function $g$ is a semianalytic function from $\chi_{0}\left(U_{0}\right)$ to $S_{n}^{2}$.

(3)(a) Let $f: S_{n}^{2} \rightarrow \mathbb{R}$ be a $C^{n}$ semianalytic function. Define $h=f \circ g: \chi_{0}\left(U_{0}\right) \rightarrow \mathbb{R}$. It is easy to check that $h$ is a semianalytic function using the fact that $f$ and $g$ are semianalytic functions. (This can be checked by using semianalytic charts on $S_{n}^{2}$, analytic ones on $\chi_{0}\left(U_{0}\right)$ and then applying Proposition A.4 and Definition A.11 of [22]).

In terms of the preferred Cartesian chart on $\chi_{0}\left(U_{0}\right)$ and any analytic chart $x^{A}$ on $S_{w}^{2}$ (which, by (2) is also a semianalytic chart on $S_{n}^{2}$ ), we may write $h \equiv f\left(x^{A}\left(x^{\alpha}\right)\right.$ ). Using the expression of Cartesian derivatives in terms of spherical ones in (4) below, it follows that $h$ is $C^{n}$. Hence $h$ is a semianalytic function from $\chi_{0}\left(U_{0}\right)$ as an analytic manifold to $S_{n}^{2}$.

(3) (b) Let us restore the semianalytic $C^{k}$ structure which $\chi_{0}\left(U_{0}\right)$ inherits from $\Sigma$. It immediately follows from (3a) that for $n \leq k, h$ is $C^{n}$ semianalytic function from $\chi_{0}\left(U_{0}\right) \subset \Sigma$ to $S_{n}^{2}$ and that for $n>k, h$ is a $C^{k}$ semianalyic function from $\chi_{0}\left(U_{0}\right) \subset \Sigma$.

(4) Start with:

$$
\left(\frac{\partial}{\partial x^{\alpha}}\right)^{a}=\stackrel{\circ}{q}^{a b} \partial_{b}\left(x_{\alpha}\right)
$$

In spherical $\left(r, x^{A}\right)$ coordinates the flat metric $\stackrel{\circ}{q}_{a b}$ takes the form:

$$
d s^{2}=d r^{2}+r^{2} q_{A B} d x^{A} d x^{B}
$$


where $q_{A B}$ is the unit sphere metric. Then in spherical coordinates $(\mathrm{A} 2)$ becomes:

$$
\begin{aligned}
\frac{\partial}{\partial x^{\alpha}} & =\partial_{r}\left(x_{\alpha}\right) \partial_{r}+r^{-2} q^{A B} \partial_{B}\left(x_{\alpha}\right) \partial_{A} . \\
& =\hat{x}_{\alpha} \partial_{r}+r^{-1} q^{A B} \partial_{B}\left(\hat{x}_{\alpha}\right) \partial_{A} .
\end{aligned}
$$

\section{Appendix B: Electric field boundary conditions in terms of fluxes}

In this appendix we present the first steps in coding the classical boundary conditions (2.1) in terms of limiting behaviour of fluxes associated with families of surfaces obtained by translations 'towards infinity' of a suitably chosen surface. The surfaces considered are all in the asymptotic region $\Sigma \backslash K$ and in the rest of this section we shall work in the preferred Cartesian chart $\left(x_{1}, x_{2}, x_{3}\right) \equiv \vec{x}$ in this region. Unit vectors are normalized with respect to the coordinate metric. We denote the unit vector along the $I$ th direction $(I=1,2,3)$ by $\hat{I}$ and the unit vector along $\vec{x}$ by $\hat{x}$ so that $\hat{x}=\frac{\vec{x}}{|\vec{x}|}$ with $|\vec{x}|=\sqrt{x_{1}^{2}+x_{2}^{2}+x_{3}^{2}}$.

For each $I=1,2,3$, let $S\left(\vec{x}_{0}, I\right)$ be the planar disc of radius $r_{0}$ centred at $\vec{x}_{0}$ with unit normal $\hat{I}$, with $\left|\vec{x}_{0}\right|, r_{0}$ chosen so that the disc is contained entirely in $\Sigma \backslash K$. Thus we have that

$$
S\left(\vec{x}_{0}, I\right)=\left\{\vec{x}: \vec{x}=\vec{x}_{0}+\vec{r},|\vec{r}| \leq r_{0}, \vec{r} \cdot \hat{I}=0\right\} .
$$

Let $S_{R}^{+}\left(\vec{x}_{0}, I\right)$ be the rigid translation of $S\left(\vec{x}_{0}, I\right)$ by the vector $\vec{R}=R \hat{x}_{0}, R>0$. Let $S_{R}^{-}\left(\vec{x}_{0}, I\right)$ be the rigid translation of $S\left(\vec{x}_{0}, I\right)$ by the vector $-2 \overrightarrow{x_{0}}-\vec{R}$ so that $S_{R}^{-}\left(\vec{x}_{0}, I\right)$ is obtained by reflecting $S_{R}^{+}\left(\vec{x}_{0}, I\right)$ through the origin while assigning its orientation such that its unit normal is also $\hat{I}$. Let $f_{I}^{i}(\vec{x}), \vec{x} \in S\left(\vec{x}_{0}, I\right)$ be the $s u(2)$ valued smearing function associated with $S\left(\vec{x}_{0}, I\right)$. Define the smearing functions $f_{ \pm, I}^{i}$ associated with $S_{R}^{ \pm}\left(\vec{x}_{0}, I\right)$ by appropriate translations of $f_{I}^{i}$ :

$$
\begin{aligned}
f_{+, I}^{i}(\vec{x}) & =f_{I}^{i}(\vec{x}-\vec{R}) \\
f_{-, I}^{i}(\vec{x}) & =f_{I}^{i}\left(\vec{x}+2 \overrightarrow{x_{0}}+\vec{R}\right) .
\end{aligned}
$$

Then it is easy to check that the fall-off conditions (2.1) on the triad field induce the following limiting behaviour on the fluxes on the above families of probes for each $I=1,2,3$ :

$$
\begin{gathered}
\lim _{R \rightarrow \infty} F_{S_{R}^{ \pm}\left(\vec{x}_{0}, I\right), f_{ \pm}, I}=\int_{S\left(\vec{x}_{0}, I\right)} d^{2} S_{I} f_{I}^{i=I}(\vec{x}), \\
\lim _{R \rightarrow \infty} R\left(F_{S_{R}^{ \pm}\left(\vec{x}_{0}, I\right), f_{ \pm, I}}-\int_{S\left(\vec{x}_{0}, I\right)} d^{2} S_{I} f_{\hat{I}}^{i=I}(\vec{x})\right)=h_{i}^{I}\left(\hat{x}_{0}\right) \int_{S\left(\vec{x}_{0}, I\right)} d^{2} S_{I} f_{I}^{i}(\vec{x}), \\
\lim _{R \rightarrow \infty} R^{1+\epsilon}\left(F_{S_{R}^{ \pm}\left(\vec{x}_{0}, I\right), f_{ \pm}^{i}, I}-\int_{S\left(\vec{x}_{0}, I\right)} d^{2} S_{I} f_{\hat{I}}^{i=I}(\vec{x})-R^{-1} h_{i}^{I}\left(\hat{x}_{0}\right) \int_{S\left(\vec{x}_{0}, I\right)} d^{2} S_{I} f_{I}^{i}(\vec{x})\right)=0,
\end{gathered}
$$

for some $\epsilon>0$. Here $d^{2} S_{I}:=d x_{J} d x_{K}, I \neq J \neq K$ and $h_{i}^{I}\left(\hat{x}_{0}\right)$ is the evaluation of the (even) function $h_{i}^{I}$ which parameterizes the next to leading order part of the triad (see equation (2.1) at the point $\hat{x}_{0}$ on the unit 2-sphere. We have used the summation convention over repeated occurrences of the $s u(2)$ index $i$ but not over the Cartesian coordinate index $I$.

It is easy to verify that the existence of $s u(2)$ valued functions $h_{I}$ on the unit 2-sphere for 
which equations (B4), (B5), (B6) are satisfied for all choices of $f_{I}^{i}, \hat{x}_{0}$ imply an asymptotic expansion of $E^{a}$ of the form:

$$
E_{i}^{\alpha}=\stackrel{\circ}{E}_{i}^{\alpha}+\frac{h_{i}^{\alpha}(\hat{x})}{r}+g_{i}^{\alpha},
$$

with $\lim _{r \rightarrow \infty} r^{1+\epsilon} g_{i}^{\alpha}=0$. This is however not the full set of conditions on $E^{a}$. For example, there is no control over the fall-offs of $\partial_{\beta} g_{i}^{\alpha}$. This requires further specifications, involving pair of surfaces approaching to each other at appropriate rates. Whereas we do not envisage obstacles in doing so, we leave for future work the determination of a full set of conditions on fluxes capturing (2.1).

We conclude by showing that conditions $(\mathrm{B} 4),(\mathrm{B} 5),(\mathrm{B} 6)$ hold in the quantum theory defined by the KS representation in the following sense. Consider any KS spinnet $|s, E\rangle$. From section III A, each such background triad state label $E_{i}^{a}$ satisfies the asymptotic conditions (2.1) for some appropriate $h_{i}^{a}(\hat{x})$. Since the spinnet graphs are confined to some compact region, it follows that for large enough $R$ the surfaces $S_{R}^{ \pm}\left(\vec{x}_{0}, I\right)$ do not intersect the graph underlying the spinnet label $s$. It follows that the standard LQG contributions to the action of flux operators associated with these surfaces vanish for sufficiently large $R$. This implies that

$$
\begin{aligned}
\hat{F}_{S_{R}^{ \pm}\left(\vec{x}_{0}, I\right), f_{ \pm, I}}|s, E\rangle & =\lambda_{E, S_{R}^{ \pm}\left(\vec{x}_{0}, I\right), f_{ \pm, I}}|s, E\rangle \\
\lambda_{E, S_{R}^{ \pm}\left(\vec{x}_{0}, I\right), f_{ \pm, I}} & =\int_{S_{R}^{ \pm}\left(\vec{x}_{0}, I\right)} d^{2} S_{a} f_{ \pm, I}^{i}(\vec{x}) E_{i}^{a}(\vec{x})
\end{aligned}
$$

so that, for sufficiently large $R$ the $\mathrm{KS}$ spinnet $|s, E\rangle$ is an eigenvector of the operators $\hat{F}_{S_{R}^{ \pm}\left(\vec{x}_{0}, I\right), f_{ \pm, I}}$. It is then easy to check that if we replace the classical fluxes in the conditions (B4), (B5) by their quantum eigenvalues (B8), these conditions hold.

\section{Appendix C: Supplementary material for section VII}

\section{Preservation of $\mathcal{A}$ and $\mathcal{E}_{E}$ by Aut $^{\stackrel{\circ}{E}}$}

Let $\phi$ and $g$ be a $C^{k}$ diffeomorphism and $C^{k-1} S U(2)$ rotation as in section VII B.

$$
\begin{aligned}
\phi(x)^{\alpha} & =R_{\beta}^{\alpha} x^{\beta}+t^{\alpha}+s^{\alpha}(\hat{x})+O\left(r^{-\epsilon}\right), \\
g(x) & =\stackrel{\circ}{g}+\frac{\lambda(\hat{x})}{r}+O\left(r^{-1-\epsilon}\right),
\end{aligned}
$$

where $s^{\alpha}$ is a $C^{k+1}$ odd function on the sphere and $\lambda$ a $C^{k}$ even function on the sphere. In this section we show that for any asymptotically flat electric field $E^{a} \in \mathcal{E}_{E}$ as in 2.1 and any connection $A_{a} \in \mathcal{A}$ as in 2.2 one has:

$$
\begin{aligned}
\phi_{*} E^{\alpha}(x) & =R_{\beta}^{\alpha} \stackrel{\circ}{E}^{\beta}+(\text { even }) / r+O\left(r^{-1-\epsilon}\right) \\
\phi_{*} A_{\alpha}(x) & =(\text { odd }) / r^{2}+O\left(r^{-2-\epsilon}\right), \\
g(x) E^{\alpha}(x) g^{-1}(x) & =\stackrel{\circ}{g} \stackrel{\circ}{\alpha}^{\alpha}{ }^{-1}+(\text { even }) / r+O\left(r^{-1-\epsilon}\right), \\
g(x) A_{\alpha}(x) g^{-1}(x)-\left(\partial_{\alpha} g(x)\right) g^{-1}(x) & =(\text { odd }) / r^{2}+O\left(r^{-2-\epsilon}\right),
\end{aligned}
$$


where "(even)" denote $C^{k}$ even functions on the sphere and "(odd)" denote $C^{k-1}$ odd functions on the sphere (from here onwards, all appearances of "(even)" and "(odd)" will refer to functions with these degrees of differentiability). Our main tool will be given by general properties of remainders described in appendix C 5 .

We first note that from appendix C $3 \phi^{-1}$ has the asymptotic expansion:

$$
\phi^{-1 \alpha}(x)=\left(R^{-1}\right)_{\beta}^{\alpha} x^{\beta}+t^{\prime \alpha}+s^{\prime \alpha}(\hat{x})+O\left(r^{-\epsilon}\right),
$$

with $t^{\prime \alpha}=-\left(R^{-1}\right)_{\beta}^{\alpha} t^{\beta}$ and $s^{\prime \alpha}(x)=-\left(R^{-1}\right)_{\beta}^{\alpha} s^{\beta}\left(R^{-1}(x)\right)$. Next we note that:

$$
\begin{aligned}
\partial_{\beta} \phi^{\alpha}(x) & =R_{\beta}^{\alpha}+(\text { even }) / r+O\left(r^{-1-\epsilon}\right), \\
\partial_{\beta} \phi^{-1 \alpha}(x) & =\left(R^{-1}\right)_{\beta}^{\alpha}+(\text { even }) / r+O\left(r^{-1-\epsilon}\right) .
\end{aligned}
$$

The Cartesian coordinate expression of $(\mathrm{C} 3)$ consists of a product of three terms:

$$
\phi_{*} E^{\alpha}(x)=\left[\operatorname{det}\left(\partial \phi^{-1}(x)\right)\right]\left[\partial_{\beta} \phi^{\alpha}\left(\phi^{-1}(x)\right)\right]\left[E^{\beta}\left(\phi^{-1}(x)\right)\right] .
$$

From (C9) and (C69) one finds: $\operatorname{det}\left(\partial \phi^{-1}(x)\right)=1+($ even $) / r+O\left(r^{-1-\epsilon}\right)$. From (C8), (C7) and Eq. (C88) one has: $\partial_{\beta} \phi^{\alpha}\left(\phi^{-1}(x)\right)=\left(R^{-1}\right)_{\beta}^{\alpha}+($ even $) / r+O\left(r^{-1-\epsilon}\right)$. Finally, from (2.1) and $\mathrm{C} 88$ one finds: $E^{\beta}\left(\phi^{-1}(x)\right)=\AA^{\beta}+($ even $) / r+O\left(r^{-1-\epsilon}\right)$. Multiplying these three expansions one obtains (C3).

The Cartesian coordinate expression of (C4) consists of a product of two terms:

$$
\phi_{*} A_{\alpha}(x)=\left[\partial_{\alpha} \phi^{-1 \beta}(x)\right]\left[A_{\beta}\left(\phi^{-1}(x)\right] .\right.
$$

The first term is given by (C9). The second term is $A_{\beta}\left(\phi^{-1}(x)\right)=($ odd $) r^{-2}+O\left(r^{-2-\epsilon}\right)$ by use of Eq. (C89). Multiplying the two terms and using Eq. (C70) one recovers (C4).

Next, taking the complex conjugate and transpose of (C2) one gets:

$$
g^{-1}(x)=\stackrel{\circ}{g}^{-1}+(\text { even }) / r+O\left(r^{-1-\epsilon}\right) .
$$

Eq. (C5) follows straightforwardly by multiplying the expansions of $g(\mathrm{C} 2), E^{a}$ (2.1) , and $g^{-1}$ (C13) and use of Eq. (C70). Similarly the linear in $A_{a}$ term in (C6) directly follows by use of Eq. (C70). The inhomogeneous term in (C6) follows by multiplying $\partial_{\alpha} g(x)=($ odd $) / r^{2}+O\left(r^{-2-\epsilon}\right)$ with (C13) and using Eq. C70.

\section{Characterization of Aut $^{\stackrel{\circ}{E}}$ as symmetry group of $\mathcal{E}_{E}$}

In this section we refer to $\operatorname{Aut}^{{ }^{\circledR}}$ as the set of pairs $\left(g^{\prime}, \phi^{\prime}\right)$ leaving the space of asymptotically flat electric fields $\mathcal{E}_{E}$ invariant:

$$
\operatorname{Aut}^{\stackrel{̊}{E}}:=\left\{\left(g^{\prime}, \phi^{\prime}\right): g^{\prime} \phi_{*}^{\prime} E^{a} g^{\prime-1} \in \mathcal{E}_{\mathscr{E}}, \quad \forall E^{a} \in \mathcal{E}_{E}\right\} .
$$

Here $g^{\prime}$ are $C^{k-1} S U(2)$ rotations and $\phi^{\prime} C^{k}$ diffeomorphisms of $\Sigma$. The purpose of the section is to show that the (component connected to identity of the) set (C14) coincides with what is referred to as $\mathrm{Aut}^{\stackrel{\bullet}{E}}$ in section VIIB. Let $\phi:=\phi^{\prime-1}$ and $g:=\phi_{*}^{\prime-1} g^{\prime-1}$ so that 
$(g, \phi)\left(g^{\prime}, \phi^{\prime}\right)=(\mathbf{1}$, Id $)$ and condition (C14) takes the form $\left(\phi^{-1}\right)_{*}\left(g^{-1} E^{a} g\right) \in \mathcal{E}_{E_{E}}$. For the particular case of an electric field $E^{a}$ that exactly coincides with the flat $E^{a}$ in the asymptotic region (for sufficiently large $r$ ), condition (C14) reads: ${ }^{27}$

$$
E^{\prime \alpha}:=\left(\phi^{-1}\right)_{*}\left(g^{-1} \stackrel{\circ}{E}^{\alpha} g\right)(x)=\stackrel{\circ}{E}^{\alpha}+(\text { even }) / r+O\left(r^{-1-\epsilon}\right) .
$$

It will be convenient to work with undensitized quantities. Taking the determinant of $E^{\prime \alpha}$ on both sides of (C15) and using Eq. (C69) one finds $\operatorname{det}\left(E^{\prime}\right)=1+($ even $) / r+O\left(r^{-1-\epsilon}\right)$. From Eq. (C72) any power of the determinant obeys similar fall-offs. Since $\operatorname{det}\left(E^{\prime}\right)=\phi_{*}^{-1} \operatorname{det}(\stackrel{\circ}{E})$ (for large enough $r$ ) one can then obtain an 'undensitized' version of (C15):

$$
e^{\prime \alpha}=\left(\phi^{-1}\right)_{*}\left(g^{-1} e^{\alpha} g\right)(x)=e^{\alpha}+(\text { even }) / r+O\left(r^{-1-\epsilon}\right),
$$

where $e^{\prime \alpha}:=\operatorname{det}^{-1 / 2}\left(E^{\prime}\right) E^{\prime \alpha}$ and $e^{\alpha}$ is the flat undensitized triad associated to $E^{\alpha}$. Setting $q^{\prime \alpha \beta}:=e_{i}^{\prime \alpha} e_{i}^{\prime \beta}$ one finds $q^{\prime \alpha \beta}=\dot{q}^{\alpha \beta}+($ even $) / r+O\left(r^{-1-\epsilon}\right)$ where $\dot{q}^{\alpha \beta}$ is the contravariant flat metric in the asymptotic region. Writing $q_{\alpha \beta}^{\prime}$ in terms of $q^{\prime \alpha \beta}$ by the standard inverse matrix formula and using (C69), (C71) one finds:

$$
q_{\alpha \beta}^{\prime}(x)=\stackrel{\circ}{q} \alpha \beta+(\text { even }) / r+O\left(r^{-1-\epsilon}\right),
$$

where $\stackrel{\circ}{\alpha \beta}_{\alpha}=\delta_{\alpha \beta}$ is the flat metric in the asymptotic region. We then conclude $\phi$ satisfies:

$$
\phi_{*}^{-1} \stackrel{\circ}{\alpha \beta}_{\alpha \beta}(x)=\stackrel{\circ}{q}_{\alpha \beta}+(\text { even }) / r+O\left(r^{-1-\epsilon}\right)=: C_{\alpha \beta}(x) .
$$

We now use $(\mathrm{C} 18)$ to determine the asymptotic form of $\phi$. In what follows we use $\stackrel{\circ}{\alpha \beta}_{\alpha}$ to rise and lower indices. Let

$$
D_{\alpha}^{\beta}(x):=\partial_{\alpha} \phi^{\beta}(x),
$$

so that from the definition of push-forward in (C18) we have:

$$
C_{\alpha \beta}(x)=D_{\alpha}^{\mu}(x) D_{\beta \mu}(x) .
$$

Eq. (C18) and its first derivative then read:

$$
\begin{aligned}
C_{\alpha \beta}(x) & =\delta_{\alpha \beta}+r^{-1} \text { (even) }+O_{k-1}\left(r^{-1-\epsilon}\right), \\
\partial_{\gamma} C_{\alpha \beta}(x) & =r^{-2} \text { (odd) }+O_{k-2}\left(r^{-2-\epsilon}\right)
\end{aligned}
$$

(subscripts in remainders denote number of derivatives with known fall-offs, see appendix C 5). For later purposes, we note that the $r^{-2}$ (odd) term in (C22) is of Cartesian degree of differentiability $k-1$ (this follows as direct consequence of the $r^{-1}$ (even) term in (C15) being of Cartesian degree of differentiability $k$ ). From the 'integrability condition' $\partial_{\gamma} \partial_{\beta} \phi^{\alpha}=$ $\partial_{\beta} \partial_{\gamma} \phi^{\alpha}$ :

$$
\partial_{\gamma} D_{\alpha \beta}(x)=\partial_{\alpha} D_{\gamma \beta}(x),
$$

${ }^{27}$ Such an electric field can be obtained by interpolating $\stackrel{\circ}{E}^{a}$ for $r>r_{2}$ with a zero electric field for $r<r_{1}<r_{2}$ through a function $f(r)$ of the type given in Eq. (A7) of [13] by $E^{a}(x)=f(r) \dot{E}^{a}$. 
one can verify the identity:

$$
\partial_{\gamma} D_{\alpha \beta}=\frac{1}{2}\left(D^{-1}\right)_{\beta}{ }^{\mu}\left(\partial_{\alpha} C_{\gamma \mu}+\partial_{\gamma} C_{\alpha \mu}-\partial_{\mu} C_{\alpha \gamma}\right),
$$

where $D^{-1}$ is the inverse matrix of $D$. Contracting indices in $\mathrm{C} 21$ we find $D^{\alpha \beta}(x) D_{\alpha \beta}(x)=$ $3+O_{0}\left(r^{-1}\right)$ which implies $D_{\alpha}^{\beta}$ is bounded. Equations similar to C20 (C21) hold for an analysis of the push forward of the contravariant metric with $D$ replaced by $D^{-1}$. As a result, we have that $D_{\alpha}^{-1} \beta$ is also bounded. This, together with Eqns. (C24) and (C22) imply:

$$
\partial_{\gamma} D_{\alpha}^{\beta}(x)=O_{0}\left(r^{-2}\right) .
$$

Let $r, \theta, \phi$ denote polar coordinates. Integration of (C25) along the radial direction implies that $\lim _{r \rightarrow \infty} D(r, \theta, \phi)=: D(\theta, \phi)$. Angular integration of (C25) at fixed $r$ followed by taking the $r \rightarrow \infty$ then implies that $D$ asymptotes to a constant matrix:

$$
R_{\alpha}^{\beta}:=\lim _{r \rightarrow \infty} D_{\alpha}^{\beta}(x) .
$$

From $(\mathrm{C} 21)$ and $(\mathrm{C} 20)$ this matrix is orthogonal. Furthermore, since $\phi$ is connected to identity, it has to be a rotation. ${ }^{28}$ It will be convenient to factor out this rotation and write $\phi$ and $D$ as:

$$
\phi^{\alpha}(x)=R_{\beta}^{\alpha}\left(x^{\beta}+y^{\beta}(x)\right), \quad D_{\alpha}^{\beta}(x)=R_{\gamma}^{\beta} \bar{D}_{\alpha}^{\gamma}(x)
$$

where

$$
\bar{D}_{\alpha}^{\beta}(x)=\delta_{\alpha}^{\beta}+Y_{\alpha}^{\beta}(x), \quad Y_{\alpha}^{\beta}(x)=\partial_{\alpha} y^{\beta}(x),
$$

with

$$
\lim _{r \rightarrow \infty} Y_{\alpha}^{\beta}(x)=0, \quad \partial_{\gamma} Y_{\alpha}^{\beta}(x)=O_{0}\left(r^{-2}\right) .
$$

The overall rotation does not affect relations $(\mathrm{C} 20)$ and $(\mathrm{C} 22)$ and one has:

$$
\begin{aligned}
C_{\alpha \beta} & =\bar{D}_{\alpha}{ }^{\mu} \bar{D}_{\beta \mu}, \\
\partial_{\gamma} Y_{\alpha \beta} \equiv \partial_{\gamma} \bar{D}_{\alpha \beta} & =\frac{1}{2}\left(\bar{D}^{-1}\right)_{\beta}{ }^{\mu}\left(\partial_{\alpha} C_{\gamma \mu}+\partial_{\gamma} C_{\alpha \mu}-\partial_{\mu} C_{\alpha \gamma}\right) .
\end{aligned}
$$

We now determine the form of $y^{\alpha}(x)$. Contracting the second equation in (C29) with $\hat{x}^{\gamma}$ and using $\partial_{r}=\hat{x}^{\gamma} \partial_{\gamma}$ one finds $\partial_{r} Y_{\alpha}{ }^{\beta}=O_{0}\left(r^{-2}\right)$. Integrating with respect to $r$ and using the first equation in $(\mathrm{C} 29)$ we find:

$$
Y_{\alpha}^{\beta}(x)=O_{0}\left(r^{-1}\right) .
$$

From the first equation in $\mathrm{C} 28$ and $\mathrm{C} 32$ it is easy to verify that:

$$
\left(\bar{D}^{-1}\right)_{\alpha}^{\beta}(x)=\delta_{\alpha}^{\beta}+O_{0}\left(r^{-1}\right) .
$$

Using (C33) and (C22) in C31 one finds:

$$
\partial_{\gamma} Y_{\beta}^{\alpha}(x)=r^{-2}(\text { odd })+O_{0}\left(r^{-2-\epsilon}\right) .
$$

${ }^{28}$ Even if we drop the connected to identity condition on $\phi$, the preservation of the triad at infinity in (C15) implies $\phi$ is orientation preserving so that $D$ can only asymptote to a rotation. 
Contracting C34 with $\hat{x}^{\gamma}$ one obtains $\partial_{r}\left(Y_{\beta}{ }^{\alpha}\right)=r^{-2}($ even $)+O_{0}\left(r^{-2-\epsilon}\right)$ so that

$$
Y_{\beta}^{\alpha}(x)=r^{-1} f_{\beta}^{\alpha}(\hat{x})+O_{1}\left(r^{-1-\epsilon}\right),
$$

where $f_{\beta}^{\alpha}$ is even. That the remainder in $(\overline{\mathrm{C} 35})$ is $O_{1}$ rather than $O_{0}$ can be seen by applying $\partial_{\gamma}$ to $(\mathrm{C} 35)$ and comparing the result with (C34). Contracting (C35) with $\hat{x}^{\beta}$ we obtain an equation for $\partial_{r} y^{\alpha}$ which upon integration over $r$ gives:

$$
y^{\alpha}(x)=\ln r f^{\alpha}(\hat{x})+\dot{y}^{\alpha}(\hat{x})+O_{2}\left(r^{-\epsilon}\right),
$$

where

$$
f^{\alpha}(\hat{x}):=\hat{x}^{\beta} f_{\beta}^{\alpha}(\hat{x})=(\text { odd }),
$$

$\dot{y}^{\alpha}(\hat{x})$ an integration 'constant' and the fact that the remainder is $O_{2}$ follows from the same logic as in (C35). In order to further determine the form of the terms in (C36), we apply $\partial_{\beta}$ to (C36) and compare the result with (C35). In spherical coordinates $\left(r, x^{A}\right)$ we have:

$$
\partial_{\beta}=r^{-1} D^{A} \hat{x}_{\beta} D_{A}+\hat{x}_{\beta} \partial_{r},
$$

where sphere indices $A, B, \ldots$ are raised with the unit sphere metric and $D_{A}$ is the derivative on the unit sphere (see Eq. (A5)). Acting on (C36) with (C38) we get:

$$
\partial_{\beta} y^{\alpha}(x)=r^{-1} \ln r D^{A} \hat{x}_{\beta} D_{A} f^{\alpha}(\hat{x})+r^{-1} \hat{x}_{\beta} f^{\alpha}(\hat{x})+r^{-1} D^{A} \hat{x}_{\beta} D_{A} \dot{y}^{\alpha}(\hat{x})+O_{1}\left(r^{-1-\epsilon}\right) .
$$

In order for $(\mathrm{C} 39)$ to be compatible with $(\mathrm{C} 35)$ it must be the case that $D^{A} \hat{x}_{\beta} D_{A} f^{\alpha}(\hat{x})=0$ which is only satisfied if $f^{\alpha}(\hat{x})=c^{\alpha}=$ constant. From the parity condition (C37) this constant must be zero and we conclude that $f^{\alpha}=0 .{ }^{29}$ Finally, for the third term in (C39) to be compatible with (C35) we must have:

$$
\grave{y}^{\alpha}(\hat{x})=t^{\alpha}+s^{\alpha}(\hat{x}),
$$

with $t^{\alpha}$ constant and $s^{\alpha}$ of odd parity. Note that from Eq. (C34) we have that $\partial_{\gamma} \partial_{\beta} s^{\alpha}$ is of Cartesian degree of differentiability $k-1$ (this differentiability coming from that in the leading order term of $(\overline{\mathrm{C} 22})$ ). Thus $s^{\alpha}(x)$ is of Cartesian degree of differentiability $k+1$ as a function on $U_{0}$. This in turn implies $s^{\alpha}(\hat{x})$ is $C^{k+1}$ as a function on the sphere.

To summarize, we have thus far shown that $\phi$ has the asymptotic form:

$$
\phi^{\alpha}(x)=R_{\beta}^{\alpha}\left(x^{\beta}+t^{\beta}+s^{\beta}(\hat{x})+y_{1}^{\beta}(x)\right),
$$

with $y_{1}^{\alpha}(x)=O_{2}\left(r^{-\epsilon}\right)$ and $s^{\alpha}(\hat{x})$ a $C^{k+1}$ odd function on the sphere. We finally show that $y_{1}^{\alpha}=O_{k}\left(r^{-\epsilon}\right)$.

If one computes $\phi_{*}^{-1} \stackrel{\circ}{\alpha \beta}_{\alpha}(x) \equiv C_{\alpha \beta}(x)$ from (C20) and (C41) one gets:

$$
C_{\alpha \beta}(x)=\delta_{\alpha \beta}+2 \partial_{(\alpha} s_{\beta)}(x)+O_{1}\left(r^{-1-\epsilon}\right) .
$$

Comparing $\mathrm{C} 42$ with $(\mathrm{C} 21)$ we conclude that the $r^{-1}$ (even) part of $C_{\alpha \beta}$ in $(\mathrm{C} 21)$ is given

${ }^{29}$ In the absence of parity conditions the resulting diffeomorphisms $x^{\alpha} \mapsto x^{\alpha}+\ln r c^{\alpha}$ are a spatial version of the so-called 'logarithmic translations' 43 . 
by $2 \partial_{(\alpha} s_{\beta}$. Eq. (C22) can then be written as:

$$
\partial_{\gamma} C_{\alpha \beta}(x)=2 \partial_{\gamma} \partial_{(\alpha} s_{\beta)}(x)+O_{k-2}\left(r^{-2-\epsilon}\right) .
$$

We now show $y_{1}^{\alpha}(x)=O_{k}\left(r^{-\epsilon}\right)$ by induction. Assume we know

$$
y_{1}^{\alpha}(x)=O_{p-1}\left(r^{-\epsilon}\right)
$$

where $3 \leq p \leq k$. We then have:

$$
\bar{D}_{\alpha}^{\beta}(x)=\delta_{\alpha}^{\beta}+\partial_{\alpha} s^{\beta}(x)+O_{p-2}\left(r^{-1-\epsilon}\right),
$$

which in turn implies:

$$
\left(\bar{D}^{-1}\right)_{\alpha}^{\beta}(x)=\delta_{\alpha}^{\beta}-\partial_{\alpha} s^{\beta}(x)+O_{p-2}\left(r^{-1-\epsilon}\right),
$$

as can be verified from the inverse matrix formula and Eqns. C69), C71). Using (C46) and (C43) in (C31) one obtains:

$$
\partial_{\gamma} Y_{\alpha \beta}(x)=\partial_{\gamma} \partial_{\alpha} s_{\beta}(x)+O_{p-2}\left(r^{-2-\epsilon}\right) .
$$

The supertranslation terms on both sides simplify and we get:

$$
\partial_{\gamma} \partial_{\beta} y_{1}^{\alpha}(x)=O_{p-2}\left(r^{-2-\epsilon}\right) .
$$

Integrating with respect to $r$ in similar way as done earlier for Eq. C34 one finds $y_{1}^{\alpha}(x)=$ $O_{p}\left(r^{-\epsilon}\right)$ as desired.

Now, recall that the diffeomorphism featuring in the definition (C14) is $\phi^{\prime} \equiv \phi^{-1}$. From appendix $\mathrm{C} 3$ one finds $\phi^{\prime}$ has the same type of expansion, with leading rotation $\left(R^{-1}\right)_{\beta}^{\alpha}$. This concludes the specification of $\phi^{\prime}$ in (C14).

We now discuss the $S U(2)$ part. Recall that we require $g$ to be such that (C15) holds. If (C15) holds then acting with $\phi_{*}$ on $(\mathrm{C} 15)$ implies that the following condition must hold:

$$
g^{-1}(x) \stackrel{\circ}{E}^{\alpha} g(x)=\phi_{*}\left(\stackrel{\circ}{E}^{\beta}+(\text { even }) / r+O\left(r^{-1-\epsilon}\right)\right) .
$$

From Eqns. (C1), (C3) of appendix C1 together with our considerations above which show that $\phi$ satisfies $\left[\mathrm{C} 1\right.$, we have that $\phi_{*}\left(\breve{E}^{\beta}+(\right.$ even $\left.) / r+O\left(r^{-1-\epsilon}\right)\right)=R_{\beta}^{\alpha} \stackrel{\circ}{ }^{\beta}+($ even $) / r+O\left(r^{-1-\epsilon}\right)$ which implies that:

$$
g^{-1}(x) \stackrel{\circ}{E}^{\alpha} g(x)=R_{\beta}^{\alpha} \stackrel{\circ}{E}^{\beta}+(\text { even }) / r+O\left(r^{-1-\epsilon}\right) .
$$

Note that the left hand side expresses the adjoint action of $g(x)$ so that it must equal $R_{\beta}^{\alpha}(x) \stackrel{\circ}{E}^{\beta}$ for some rotation matrix $R_{\beta}^{\alpha}(x)$. Conversely from the 2 to 1 map from $S U(2)$ to $S O(3), R_{\beta}^{\alpha}(x)$ uniquely specifies $g(x)$ upto a sign. Since $\lim _{r \rightarrow \infty} R_{\beta}^{\alpha}(x)=R_{\beta}^{\alpha}$ and since $g(x)$ is continuous in $x^{\alpha}$, we have that $\lim _{r \rightarrow \infty} g(x)=\stackrel{\circ}{g}$ with $\stackrel{g}{g}$ being the constant $S U(2)$ rotation satisfying $\stackrel{\circ}{g}^{-1} \stackrel{\circ}{E}^{\alpha} \stackrel{\circ}{g}=R_{\beta}^{\alpha} \stackrel{\circ}{E}^{\beta}$. From $(\mathrm{C} 50)$ it is then straightforward to conclude that $g(x)$ must be of the form

$$
g(x)=\stackrel{\circ}{g}+\lambda(\hat{x}) / r+O_{0}\left(r^{-1-\epsilon}\right),
$$

with $\lambda$ an even function on the sphere. By comparing derivatives of (C50) and (C51) one can 
conclude that the remainder term in $\mathrm{C} 51$ is $O\left(r^{-1-\epsilon}\right)$. By comparing the $r^{-1}$ (even) term in (C51) with the one in (C50) one concludes it is of Cartesian degree of differentiability $k$, which in turn implies $\lambda(\hat{x})$ is $C^{k}$ as a function on the sphere. Thus $g(x)$ is an $S U(2)$ rotation as in section VIIB. This directly implies $g^{-1}(x)$ is of the same type (see Eq. (C13)). Finally from Eq. (C74) one finds that $g^{\prime}=\phi_{*}^{-1} g^{-1}$ is also of the form given in VIIB, with leading term $\stackrel{\circ}{g}^{\prime} \equiv \stackrel{\circ}{g}^{-1}$. This concludes the characterization of $\mathrm{Aut}^{\stackrel{\circ}{E}}$ elements.

\section{Asymptotic form of $\phi^{-1}$}

Given the asymptotic form of $\phi \equiv \phi^{\prime-1}$ :

$$
\phi(x)^{\alpha}=R_{\beta}^{\alpha}\left(x^{\beta}+t^{\beta}+s^{\beta}(\hat{x})\right)+O_{k}\left(r^{-\epsilon}\right),
$$

we want to find the asymptotic form of $\phi^{\prime}=\phi^{-1}$. We first show that C52 implies:

$$
\phi^{\alpha}(x)=\left(R^{-1}\right)_{\beta}^{\alpha} x^{\beta}+O_{0}(1) .
$$

From (C52) we have that there exists $r_{0}, M>0$ s.t. $\forall r>r_{0}$ we have that

$$
|\phi(x)-R(x)|<M .
$$

$\phi$ is a homeomorphism of $\Sigma$ so that $\phi\left(B\left(r_{0}\right)\right)$ is closed and $\phi\left(\partial B\left(r_{0}\right)\right)=\partial \phi\left(B\left(r_{0}\right)\right)$ where $B\left(r_{0}\right)$ is the interior of the sphere of cartesian radius $r_{0}$ together with its boundary $\partial B\left(r_{0}\right)$. Since for $r>r_{0}$ we have that $\phi(x)$ is also in the asymptotic region, there exists $r_{0}^{\prime}$ such that $\phi\left(B\left(r_{0}\right)\right) \subset B\left(r_{0}^{\prime}\right)$. This means that if $|\phi(x)|>r_{0}^{\prime}$ then $r>r_{0}$ so that for $|\phi(x)|>r_{0}^{\prime}$ we have from equation (C54) that $|\phi(x)-R(x)|<M$ which is the same as the statement that for $|x|>r_{0}^{\prime}$, we have that $\left|x-R\left(\phi^{-1}(x)\right)\right|<M$ which is same as $\left|R^{-1}(x)-\phi^{-1}(x)\right|<M$ which means that $\phi^{-1}(x)=R^{-1}(x)+O_{0}(1)$.

Let $D_{\beta}^{\alpha}(x)=\partial_{\beta} \phi^{\alpha}(x)$ so that

$$
D_{\beta}^{\alpha}(x)=R_{\gamma}^{\alpha}\left(\delta_{\beta}^{\gamma}+\partial_{\beta} s^{\alpha}(x)\right)+O_{k-1}\left(r^{-1-\epsilon}\right) .
$$

From inverse matrix formula and Eqns. (C69), (C71) one finds:

$$
\left(D^{-1}\right)_{\beta}^{\alpha}(x)=\left(R^{-1}\right)_{\beta}^{\gamma}\left(\delta_{\gamma}^{\alpha}-\partial_{\gamma} s^{\alpha}(x)\right)+O_{k-1}\left(r^{-1-\epsilon}\right) .
$$

The inverse derivative formula tells:

$$
\partial_{\alpha} \phi^{\prime \beta}(x)=\left(D^{-1}\right)_{\alpha}^{\beta}\left(\phi^{\prime}(x)\right) .
$$

Substituting $(\mathrm{C} 53)$ in the RHS of $\overline{\mathrm{C} 57)}$ and using that $O_{0}\left(\left|\phi^{\prime}(x)\right|^{-1-\epsilon}\right)=O_{0}\left(r^{-1-\epsilon}\right)$ and $\partial_{\gamma} s^{\alpha}\left(\phi^{\prime}(x)\right)=\partial_{\gamma} s^{\alpha}\left(R^{-1}(x)\right)+O_{0}\left(r^{-2}\right)$ by Taylor expansion (see footnote 31 ) one finds:

$$
\partial_{\alpha} \phi^{\prime \beta}(x)=\left(R^{-1}\right)_{\beta}^{\gamma}\left(\delta_{\gamma}^{\alpha}-\partial_{\gamma} s^{\alpha}\left(R^{-1}(x)\right)\right)+O_{0}\left(r^{-1-\epsilon}\right)
$$

which upon integration leads to:

$$
\phi^{\prime \alpha}(x)=\left(R^{-1}\right)_{\beta}^{\alpha} x^{\beta}-s^{\alpha}\left(R^{-1}(x)\right)+c^{\alpha}+O_{1}\left(r^{-\epsilon}\right)
$$


where $c^{\alpha}$ are integration constants. The condition $\phi^{\prime}(\phi(x))=\operatorname{Id}$ fixes $c^{\alpha}=-t^{\alpha}$. That the remainder in (C59) is $O_{k}\left(r^{-\epsilon}\right)$ can be shown by induction using Eq. (C57): Assume the remainder is $O_{m-1}\left(r^{-\epsilon}\right), 2 \leq m \leq k$ for $\phi^{\prime}$ on the RHS of (C57) and compute $\partial_{\alpha} \phi^{\prime \beta}$ from this equation. Using Eq. C88) with $\left(D^{-1}\right)_{\beta}^{\alpha}(x)$ seen as the $C^{k-1}$ function (C56) and $\phi^{\prime}$ seen as a $C^{m-1}$ diffeomorphism, one obtains $\partial_{\alpha} \phi^{\prime \beta}(x)=\left(R^{-1}\right)_{\beta}^{\gamma}\left(\delta_{\gamma}^{\alpha}-\partial_{\gamma} s^{\alpha}\left(R^{-1}(x)\right)\right)+O_{m-1}\left(r^{-1-\epsilon}\right)$ which implies the remainder of $\phi^{\prime}$ is $O_{m}\left(r^{-\epsilon}\right)$. ${ }^{30}$

\section{Asymptotic form of Aut ${ }^{\stackrel{\circ}{E}}$ compositions}

Let $(g, \phi),\left(g^{\prime}, \phi^{\prime}\right) \in \mathrm{Aut}^{\stackrel{\circ}{E}}$ as in 7.11 and $\left(g^{\prime \prime}, \phi^{\prime \prime}\right):=(g, \phi)(g, \phi)=\left(g \phi_{*} g^{\prime}, \phi \phi^{\prime}\right)$. Definition C14 implies $\left(g^{\prime \prime}, \phi^{\prime \prime}\right) \in$ Aut $^{E}$ so that from section $\mathrm{C} 2$ it has also the asymptotic form:

$$
\begin{gathered}
\phi^{\prime \prime \alpha}(x)==R_{\beta}^{\alpha}\left(\stackrel{\circ}{g}^{\prime \prime}\right) x^{\beta}+t^{\prime \prime \alpha}+s^{\prime \prime \alpha}(\hat{x})+O\left(r^{-\epsilon}\right), \\
g^{\prime \prime}(x)=\stackrel{\circ}{g}^{\prime \prime}+r^{-1}(\text { even })+O\left(r^{-1-\epsilon}\right),
\end{gathered}
$$

where $R_{\beta}^{\alpha}: S U(2) \rightarrow S O(3)$ denotes the Adjoint action of $S U(2)$ defined by Eq. (7.10). From $\phi^{\prime \prime}=\phi \phi^{\prime}$ and using properties as in appendix $\mathrm{C} 5$ one finds

$$
\begin{aligned}
R_{\beta}^{\alpha}\left(\stackrel{\circ}{g}^{\prime \prime}\right) & =R_{\mu}^{\alpha}(\stackrel{\circ}{g}) R_{\beta}^{\mu}\left(\stackrel{\circ}{g}^{\prime}\right), \\
t^{\prime \prime \alpha} & =R_{\mu}^{\alpha}(\stackrel{\circ}{g}) t^{\prime \mu}+t^{\alpha}, \\
s^{\prime \prime \alpha}(x) & =R_{\mu}^{\alpha}(\stackrel{\circ}{g}) s^{\prime \mu}(x)+s^{\alpha}\left(R\left(\stackrel{\circ}{g}^{\prime}\right) x\right) .
\end{aligned}
$$

From $g^{\prime \prime}=g \phi_{*} g^{\prime}$ and the properties of appendix C 5 one finds $\stackrel{\circ}{g}^{\prime \prime}=\stackrel{\circ}{g} \stackrel{\circ}{ }^{\prime}$. This is consistent with C62 since $R_{\gamma}^{\alpha}(\stackrel{\circ}{g}) R_{\beta}^{\gamma}\left(\stackrel{\circ}{g}^{\prime}\right)=R_{\beta}^{\alpha}\left(\stackrel{\circ}{g} \stackrel{g}{ }^{\prime}\right)$.

From these properties it follows that the set of Aut $^{\stackrel{\circ}{E}}$ elements with trivial asymptotic rotations and translations form a subgroup of Aut $^{E}$. The group Aut is defined as the component connected to identity of this subgroup (one can verify that the connected to identity property as defined in footnote 15 is preserved under the group operations).

\section{Properties of remainders}

A $C^{p}$ field is said to be $O_{n}\left(r^{-\beta}\right)(0 \leq n \leq p \leq k ; \beta \geq 0)$ if for $m=0, \ldots, n$ the coefficients of the $m$-th partial derivatives in the Cartesian chart are bounded by $\mathrm{cr}^{-\beta-m}$ for some constant $c$. The case when $n=p$ is denoted by $O\left(r^{-\beta}\right)$ as presented in section II B. Product of remainders satisfies:

$$
O_{m}\left(r^{-\beta}\right) O_{n}\left(r^{-\gamma}\right)=O_{\min (m, n)}\left(r^{-\beta-\gamma}\right),
$$

30 The last induction step $m=k$ makes use of the $C^{k+1}$ Cartesian differentiability of $s^{\alpha}$ (in its role of $\partial_{\gamma} s^{\alpha}$ term in (C56) by the same reason described in footnote 32 
as can be verified by taking derivatives on the left hand side. We now describe the behaviour under various operations of functions of the form:

$$
\begin{aligned}
& f(x)=f_{0}+f_{1}(x)+O\left(r^{-1-\epsilon}\right), \\
& a(x)=a_{2}(x)+O\left(r^{-2-\epsilon}\right),
\end{aligned}
$$

where $f$ is $C^{p}, a$ is $C^{p^{\prime}}, 0 \leq p, p^{\prime} \leq k, f_{0}=$ constant and

$$
f_{1}(x)=g(\hat{x}) / r, \quad a_{2}(x)=b(\hat{x}) / r^{2},
$$

with $g(\hat{x}), b(\hat{x})$ respectively $C^{p+1}, C^{p^{\prime}+1}$, functions on the sphere.

We first consider the product of functions: Given $f(x), a(x)$ and $f^{\prime}(x)=f_{0}^{\prime}+f_{1}^{\prime \prime}(x)+$ $O\left(r^{-1-\epsilon}\right)$ as in (C66), it is easy to verify that their product is again of the type (C66), (C67) with:

$$
\begin{aligned}
f(x) f^{\prime}(x) & =f_{0} f_{0}^{\prime}+\left(f_{0} f_{1}^{\prime}(x)+f_{0}^{\prime} f_{1}(x)\right)+O\left(r^{-1-\epsilon}\right), \\
f(x) a(x) & =f_{0} a_{2}(x)+O\left(r^{-2-\epsilon}\right) .
\end{aligned}
$$

Next we note that when $f_{0} \neq 0$ :

$$
1 / f(x)=f_{0}^{-1}-f_{0}^{-2} f_{1}(x)+O\left(r^{-1-\epsilon}\right) .
$$

To see (C71), use Taylor expansion around $t=1$ of the function $t \mapsto 1 / t$ to obtain: $1 / f(x)=$ $f_{0}^{-1}-f_{0}^{-2} f_{1}(x)+O_{0}\left(r^{-1-\epsilon}\right)$. The appropriate bounds on the derivatives of the remainders can be obtained by induction as follows. Assume $1 / f(x)=f_{0}^{-1}-f_{0}^{-2} f_{1}(x)+O_{n}\left(r^{-1-\epsilon}\right)$. Writing $\partial_{\alpha}(1 / f(x))=-(1 / f(x))^{2} \partial_{\alpha} f(x)$ and using (C65) one concludes that the derivative of the remainder is $O_{n}\left(r^{-2-\epsilon}\right)$. This in turn implies the remainder is $O_{n+1}\left(r^{-1-\epsilon}\right)$.

More generally one can verify that:

$$
(f(x))^{\lambda}=f_{0}^{\lambda}+\lambda f_{0}^{\lambda-1} f_{1}(x)+O\left(r^{-1-\epsilon}\right),
$$

for any $\lambda \in \mathbb{R}$ and $f_{0} \neq 0$. This can again be shown by induction: Assume $f^{\lambda}(x)=$ $f_{0}^{\lambda}+\lambda f_{0}^{\lambda-1} f_{1}(x)+O_{n}\left(r^{-1-\epsilon}\right)\left(n=0\right.$ case is obtained by Taylor expansion of $\left.t \mapsto t^{\lambda}\right)$. From $\partial_{\alpha}\left(f^{\lambda}(x)\right)=\lambda\left(f^{\lambda}(x)\right)(1 / f(x)) \partial_{\alpha} f(x)$ and using (C65) and C71) one concludes that the derivative of the remainder is $O_{n}\left(r^{-2-\epsilon}\right)$, which in turn implies the remainder is $O_{n+1}\left(r^{-1-\epsilon}\right)$.

We now describe the behaviour of functions (C66), C67) under composition with diffeomorphisms. To simplify the discussion we first consider diffeomorphisms with asymptotic trivial rotational part. Consider a $C^{l}, 0 \leq l \leq k$ diffeomorphism with asymptotic form

$$
\phi(x)^{\alpha}=x^{\alpha}+\dot{\circ}^{\alpha}(\hat{x})+O\left(r^{-\epsilon}\right)
$$

with $\grave{y}^{\alpha}(\hat{x})$ a $C^{l+1}$ function on the sphere (the case $l=k, \stackrel{\circ}{y}^{\alpha}(\hat{x})=t^{\alpha}+s^{\alpha}(\hat{x})$ corresponds to the diffeomorphisms of section C2. For $f$ as in (C66) we have:

$$
f(\phi(x))=f_{0}+f_{1}(x)+O\left(r^{-1-\epsilon}\right) .
$$


To show (C74) we need to verify that:

$$
\begin{aligned}
f(\phi(x)) & =f_{0}+f_{1}(x)+O_{0}\left(r^{-1-\epsilon}\right) \\
\partial_{\beta_{n}} \ldots \partial_{\beta_{1}} f(\phi(x)) & =\partial_{\beta_{n}} \ldots \partial_{\beta_{1}} f_{1}(x)+O_{0}\left(r^{-(n+1)-\epsilon}\right), \quad n=1, \ldots, \min (l, p) .
\end{aligned}
$$

$(\min (l, p)$ is the differentiability of $f \circ \phi)$. First, notice that argumentation similar to that after Eq. C53 implies that diffeomorphisms (C73) do not change $O_{0}\left(r^{-\beta}\right)$ bounds:

$$
F(x)=O_{0}\left(|x|^{-\beta}\right) \Longleftrightarrow F(x)=O_{0}\left(|\phi(x)|^{-\beta}\right),
$$

where $|\phi(x)|:=\sqrt{\phi^{\alpha}(x) \phi^{\alpha}(x)}$ and $|x| \equiv r$. We thus have:

$$
f(\phi(x))=f_{0}+f_{1}(\phi(x))+O_{0}\left(r^{-1-\epsilon}\right) .
$$

Taylor expanding $f_{1}(\phi(x))$ around $x$ we find: ${ }^{31}$

$$
f_{1}(\phi(x))=f_{1}(x)+O_{0}\left(r^{-2}\right) .
$$

From (C78) and (C79) we conclude (C75). To show $(\mathrm{C} 76)$ we use chain rule formula to take the $n$-th partial derivative of $f(\phi(x))$,

$$
\partial_{\beta_{n}} \ldots \partial_{\beta_{1}} f(\phi(x))=\sum_{m=1}^{n} C_{\beta_{n} \ldots \beta_{1}}^{\gamma_{m} \ldots \gamma_{1}}(x)\left(\partial_{\gamma_{m}} \ldots \partial_{\gamma_{1}} f\right)(\phi(x)),
$$

where:



where the sum is over all distinct partitions $I_{1}, \ldots, I_{m}$ of $\{1, \ldots, n\}$ with $I_{k}=\left\{i_{I_{k}}^{1}, \ldots, i_{I_{k}}^{j_{k}}\right\}$, $i_{I_{k}}^{1}<i_{I_{k}}^{2}<\ldots<i_{I_{k}}^{j_{k}}$ (thus $j_{k}$ is the cardinality of $I_{k}$ and $j_{1}+\ldots+j_{m}=n$ ). The formula (C80), (C81) is a realization to the present case of an abstract chain rule formula described in appendix A of [42].

From (C66) and $\mathrm{C} 77$ we have the following rough bound:

$$
\left(\partial_{\gamma_{m}} \ldots \partial_{\gamma_{1}} f\right)(\phi(x))=O_{0}\left(r^{-(m+1)}\right) .
$$

Using (C82) and the following rough bounds on derivatives of $\phi$ :

$$
\partial \phi(x)=O_{0}(1), \quad \partial^{n} \phi(x)=O_{0}\left(r^{-n}\right), n>1,
$$

one can verify that all $m<n$ terms in C80 are $O_{0}\left(r^{-(n+2)}\right)$ whereas the $m=n$ one is $O_{0}\left(r^{-(n+1)}\right)$. It then follows that:

$$
\partial_{\beta_{n}} \ldots \partial_{\beta_{1}} f(\phi(x))=\left(\partial_{\beta_{n}} \ldots \partial_{\beta_{1}} f\right)(\phi(x))+O_{0}\left(r^{-(n+2)}\right)
$$

$\overline{{ }^{31} \text { Let } x^{\prime \alpha}}:=\phi^{\alpha}(x), r^{\prime}:=|\phi(x)|$ and $\hat{x}^{\prime \alpha}=x^{\alpha} / r^{\prime}$. Using that $x^{\prime}=x+O_{0}(1)$ it is easy to verify using Taylor approximation that $r^{-1}=r^{-1}\left(1+O_{0}\left(r^{-1}\right)\right)$ and $\hat{x}^{\prime}=\hat{x}+O_{0}\left(r^{-1}\right)$. The latter implies $g\left(\hat{x}^{\prime}\right)=$ $g(\hat{x})+O_{0}\left(r^{-1}\right)$. Using these expansions for $f_{1}\left(x^{\prime}\right)=r^{\prime-1} g\left(\hat{x}^{\prime}\right)$ one obtains $\mathrm{C} 79$. Analogous considerations for functions of the form $f_{n}(x)=r^{-n} g(\hat{x}), n>0$ leads to $f_{n}\left(x^{\prime}\right)=f_{n}(x)+O_{0}\left(r^{-(n+1)}\right)$. 
where we have used that for $m=n$, Eq. C81 reduces to: $C_{\beta_{n} \ldots \beta_{1}}^{\gamma_{n} \ldots \gamma_{1}}=\partial_{\beta_{n}} \phi^{\gamma_{n}} \ldots \partial_{\beta_{1}} \phi^{\gamma_{1}}$. Using (C66) for $f$ in the right hand side of C84 we obtain

$$
\partial_{\beta_{n}} \ldots \partial_{\beta_{1}} f(\phi(x))=\left(\partial_{\beta_{n}} \ldots \partial_{\beta_{1}} f_{1}\right)(\phi(x))+O_{0}\left(r^{-(n+1)-\epsilon}\right) .
$$

Finally, Taylor expanding the first term in C85 around $x$ we find: ${ }^{32}$

$$
\left(\partial_{\beta_{n}} \ldots \partial_{\beta_{1}} f_{1}\right)(\phi(x))=\left(\partial_{\beta_{n}} \ldots \partial_{\beta_{1}} f_{1}\right)(x)+O_{0}\left(r^{-(n+2)}\right) .
$$

Using (C86) in (C85) we obtain the desired result (C76). For later use in appendix D we note that the proof goes through if $p=l=k=\infty$, so that the analogue of equation (C74) still holds in the smooth setting. The only difference is that in this case the $O_{0}\left(r^{-(n+1)-\epsilon}\right)$ bounds in (C76) are in general of the form $c_{n} r^{-(n+1)-\epsilon}$ with $n$-dependent constants $c_{n}$.

This result can be used to show that the composition of (C66) with a $C^{l}$ diffeomorphism with nontrivial rotation

$$
\phi(x)^{\alpha}=R_{\beta}^{\alpha}\left(x^{\beta}+\stackrel{\circ}{y}^{\beta}(\hat{x})\right)+O\left(r^{-\epsilon}\right)
$$

is given by

$$
f(\phi(x))=f_{0}+f_{1}(R(x))+O\left(r^{-1-\epsilon}\right),
$$

where $R(x)^{\alpha} \equiv R_{\beta}^{\alpha} x^{\beta}$. To see $\left(\right.$ C88), write $\phi^{\alpha}(x)=R_{\beta}^{\alpha} \psi^{\beta}(x)$ with $\psi^{\alpha}(x):=\left(R^{-1}\right)_{\beta}^{\alpha} \phi^{\alpha}(x)$. Next, note that $F(x)=O\left(r^{-\beta}\right) \Longleftrightarrow F(R(x))=O\left(r^{-\beta}\right)$. It follows that $f(R(x))=$ $f_{0}+f_{1}(R(x))+O\left(r^{-1-\epsilon}\right)$. Using $\mathrm{C} 74$ for $f(R(x))$ in place of $f(x)$ and $\psi^{\alpha}(x)$ in place of $\phi^{\alpha}(x)$ one obtains $(\mathrm{C} 88)$, where it is easily verified that $f_{1}(R(x))$ satisfies the same conditions of the type (C68) which are satisfied by $f_{1}(x)$.

We finally show that for $\phi$ as in (C87) and $a(x)$ as in (C67) one has:

$$
a(\phi(x))=a_{2}(R(x))+O\left(r^{-2-\epsilon}\right) .
$$

This can be seen by writing $a(x)=[r(x) a(x)][1 / r(x)], r(x) \equiv \sqrt{x^{\alpha} x^{\alpha}}$, and noting that: The function $f(x):=r(x) a(x)$ is of the form (C66) (with $f_{0}=0$ ), and so it satisfies (C88); $1 / r(x)$ is also of the form (C66) (with $f_{0}=0$ and $f_{1}=r^{-1}$ ) and so $1 / r(\phi(x))=1 / r+O\left(r^{-1-\epsilon}\right)$. Substituting these expansions in

$$
a(\phi(x))=[r(\phi(x)) a(\phi(x))][1 / r(\phi(x))]
$$

one obtains (C89).

\section{Appendix D: Phase space description of gauge and asymptotic symmetry group}

In this appendix we study the classical phase space description of gauge and asymptotic symmetries. After reviewing the infinitesimal generators as described in [19], we study the corresponding finite transformations. As in [17 [19], we restrict attention to $C^{\infty}$ fields.

32 See footnote 31 . When $p \leq l$, the condition $g(\hat{x})$ being $C^{p+1}$ is used to obtain the $n=p$ case of C86. 


\section{Infinitesimal transformations}

In standard phase space description, the kinematical gauge transformations are generated by the Gauss and diffeomorphism constraints:

$$
G[\Lambda]:=-\int_{\Sigma} \operatorname{Tr}\left[\Lambda\left(\partial_{a} E^{a}+\left[A_{a}, E^{a}\right]\right)\right], \quad D[\xi]:=\int_{\Sigma} \operatorname{Tr}\left[E^{a} \mathcal{L}_{\xi} A_{a}\right] .
$$

The fall-off conditions

$$
\Lambda^{i}=\frac{\lambda^{i}(\hat{x})}{r}+O\left(r^{-1-\epsilon}\right), \quad \xi^{\alpha}=S^{\alpha}(\hat{x})+O\left(r^{-\epsilon}\right),
$$

with $\lambda(\hat{x})$ even and $S^{\alpha}(\hat{x})$ odd ensure the well-definedness of the constraints as phase space functions. Their action is then given by:

$$
\begin{aligned}
(\Lambda, \xi) \cdot A_{a}:=\left[\Lambda, A_{a}\right]-\mathcal{L}_{\xi} A_{a}-\partial_{a} \Lambda & =\left\{G[\Lambda]+D[\xi], A_{a}\right\}, \\
(\Lambda, \xi) \cdot E^{a}:=\left[\Lambda, E^{a}\right]-\mathcal{L}_{\xi} E^{a} & =\left\{G[\Lambda]+D[\xi], E^{a}\right\},
\end{aligned}
$$

and their commutator evaluates to:

$$
\left[(\Lambda, \xi),\left(\Lambda^{\prime}, \xi^{\prime}\right)\right]=\left(-\mathcal{L}_{\xi} \Lambda^{\prime}+\mathcal{L}_{\xi^{\prime}} \Lambda+\left[\Lambda, \Lambda^{\prime}\right],-\left[\xi, \xi^{\prime}\right]\right),
$$

which corresponds to a representation of the Poisson bracket algebra of the constraints (D1). Equations (D3), (D4) represent the infinitesimal version of (7.2) and (7.7) respectively. In the case of asymptotic symmetries, the fall-off of $S U(2)$ multiplier and shift are:

$$
\begin{aligned}
& \Lambda^{i}=\Lambda_{R}^{i}+\frac{\lambda^{i}(\hat{x})}{r}+O_{2}\left(r^{-1-\epsilon}\right), \\
& \xi^{\alpha}=T^{\alpha}+R^{\alpha}+S^{\alpha}(\hat{x})+O_{2}\left(r^{-\epsilon}\right),
\end{aligned}
$$

where

$$
\Lambda_{R}^{i}:=\frac{1}{2} \epsilon_{i j k} \stackrel{\circ}{E}_{a}^{j} \mathcal{L}_{R} \stackrel{\circ}{E}_{k}^{a} .
$$

The phase space action of these generators is given by the same expressions as in the Aut case, Eq. (D3), and the commutator of generators by (D4). This action is generated via Poisson brackets by well-defined phase space functions representing the total linear and angular momenta of the spacetime [18, 19].

\section{Finite transformations}

We now describe the asymptotic form of the finite transformations associated to the above infinitesimal generators. We proceed in steps: 


\section{a. Flow equations}

Given a pair $(\Lambda, \xi)$ as in (D5), (D6) we wish to solve the 'flow equation' $d a_{t} / d t=(\Lambda, \xi) a_{t}$. Writing $a_{t}=\left(g_{t}, \phi_{t}\right)$ and assuming the composition law (7.7) this translates into:

$$
\begin{aligned}
\frac{d}{d t} \phi_{t}^{a} & =\xi^{a}\left(\phi_{t}\right), \\
\frac{d}{d t} g_{t} & =\Lambda g_{t}-\mathcal{L}_{\xi} g_{t} .
\end{aligned}
$$

Conversely, it is easy to verify that the flow equations (D8) and (D9) together with (7.7) yield the infinitesimal transformations of section D 1 so that (7.7) is indeed a finite version of these infinitesimal transformations.

\section{b. Asymptotic form of diffeomorphisms (D8)}

We show the diffeomorphisms $\phi_{t}$ obtained by integrating (D8) have asymptotic form as in 7.12 . Define the following vector fields in the asymptotic region:

$$
\stackrel{\circ}{\xi}^{\alpha}:=T^{\alpha}+R^{\alpha}, \quad Y^{\alpha}:=\xi^{\alpha}-\stackrel{\circ}{\xi}^{\alpha}, \quad \delta^{\alpha}:=Y^{\alpha}-S^{\alpha},
$$

so that

$$
\begin{aligned}
\xi^{\alpha} & =\stackrel{\circ}{\xi}^{\alpha}+Y^{\alpha}, \\
Y^{\alpha} & =S^{\alpha}+\delta^{\alpha}, \\
\delta^{\alpha} & =O\left(r^{-\epsilon}\right) .
\end{aligned}
$$

Let $\dot{\phi}_{t}$ be the flow of $\stackrel{\circ}{\xi}^{\alpha}$ and define

$$
\begin{aligned}
\psi_{t} & :=\dot{\phi}_{t}^{-1} \circ \phi_{t} \\
Y_{t}^{\alpha} & :=\stackrel{\circ}{t *}_{t}^{-1} Y^{\alpha} .
\end{aligned}
$$

It is easy to verify that $\psi_{t}$ is the flow of the $t$-dependent vector field $Y_{t}^{\alpha}$, that is, $\psi_{t=0}(x)=x$ and

$$
\frac{d}{d t} \psi_{t}^{\alpha}(x)=Y_{t}^{\alpha}\left(\psi_{t}(x)\right)
$$

Indeed, one has:

$$
\begin{aligned}
\frac{d}{d t} \dot{\phi}_{t}^{-1}\left(\phi_{t}(x)\right)^{\alpha} & =-\dot{\xi}^{\alpha}\left(\dot{\phi}_{t}^{-1}\left(\phi_{t}(x)\right)\right)+\frac{\partial\left(\dot{\phi}_{t}^{-1}\right)^{\alpha}}{\partial y^{\beta}}\left(\phi_{t}(x)\right) \xi^{\beta}\left(\phi_{t}(x)\right) \\
& =-\dot{\xi}^{\alpha}\left(\psi_{t}(x)\right)+\left(\dot{\phi}_{t *}^{-1} \xi\right)^{\alpha}\left(\psi_{t}(x)\right) \\
& =\left(\dot{\phi}_{t *}^{-1} Y\right)^{\alpha}\left(\psi_{t}(x)\right),
\end{aligned}
$$

where in going from the first to second line we used the local coordinate expression for the push-forward of vector fields: $\left(\phi_{*} X\right)^{\alpha}(x)=\partial \phi^{\alpha} / \partial y^{\beta}\left(\phi^{-1}(x)\right) X^{\beta}\left(\phi^{-1}(x)\right)$, and in going to the last line we used that $\dot{\circ}_{t *}^{-1} \stackrel{\circ}{\xi}^{a}=\stackrel{\circ}{\xi}^{a}$. 
$\stackrel{\circ}{\phi}_{t}$ is given by:

$$
\dot{\phi}_{t}(x)=R_{t}(x)+c_{t},
$$

with $R_{t}(x) \equiv\left(R_{t}\right)_{\beta}^{\alpha} x^{\beta}$ the rotation generated by $R^{\alpha}$ (so that $\frac{d}{d t} R_{t}(x)^{\alpha}=R^{\alpha}\left(R_{t}(x)\right)$ ) and $c_{t}^{\alpha}=\left(R_{t}\right)_{\beta}^{\alpha} \int_{0}^{t}\left(R_{t^{\prime}}^{-1}\right)_{\gamma}^{\beta} T^{\gamma} d t^{\prime}$ the translation piece. The nontrivial result we will show is that:

$$
\psi_{t}^{\alpha}(x)=x^{\alpha}+s_{t}^{\alpha}(\hat{x})+O\left(r^{-\epsilon}\right)
$$

with $s_{t}^{\alpha}(\hat{x})$ odd. From (D21), (D20), (D14) the desired result (7.12) directly follows. Condition (D21) can be expressed as:

$$
\begin{aligned}
\psi_{t}^{\alpha}(x) & =x^{\alpha}+s_{t}^{\alpha}(\hat{x})+O_{0}\left(r^{-\epsilon}\right) \\
\partial_{\beta_{n}} \ldots \partial_{\beta_{1}} \psi_{t}^{\alpha}(x) & =\partial_{\beta_{n}} \ldots \partial_{\beta_{1}}\left(x^{\alpha}+s_{t}^{\alpha}(\hat{x})\right)+O_{0}\left(r^{-n-\epsilon}\right), \quad n=1,2, \ldots,
\end{aligned}
$$

where $O_{0}\left(r^{-\gamma}\right)$ denotes bounded by $c r^{-\gamma}$ for some constant $c$. We first show (D22) and then provide an induction argument for (D23). The integrated version of (D16) reads:

$$
\psi_{t}(x)^{\alpha}=x^{\alpha}+\int_{0}^{t} Y_{t^{\prime}}^{\alpha}\left(\psi_{t^{\prime}}(x)\right) d t^{\prime} .
$$

Let

$$
y_{t}^{\alpha}(x):=\int_{0}^{t} Y_{t^{\prime}}^{\alpha}\left(\psi_{t^{\prime}}(x)\right) d t^{\prime}
$$

Boundedness of $Y^{\alpha}$ implies boundedness of $Y_{t}^{\alpha}$ which in turn implies implies boundedness of $y_{t}^{\alpha}(x)$. Thus $\psi_{t}(x)$ stays in the asymptotic region and $y_{t}^{\alpha}(x)=O_{0}\left(r^{0}\right)$ :

$$
\begin{aligned}
\psi_{t}^{\alpha}(x) & =x^{\alpha}+y_{t}^{\alpha}(x) \\
& =x^{\alpha}+O_{0}\left(r^{0}\right) .
\end{aligned}
$$

We now use (D27) to determine the asymptotic form of the integrand in (D24):

$$
Y_{t}^{\alpha}\left(\psi_{t}(x)\right)=\stackrel{\circ}{\phi *}_{t *}^{-1} S^{\alpha}\left(\psi_{t}(x)\right)+\stackrel{\circ}{\phi}_{t *}^{-1} \delta^{\alpha}\left(\psi_{t}(x)\right) .
$$

For the first term in (D28) we have:

$$
\begin{aligned}
\dot{\phi}_{t *}^{-1} S^{\alpha}\left(\psi_{t}(x)\right) & =\left(R_{t}^{-1}\right)_{\beta}^{\alpha} S^{\beta}\left(R_{t}(x)+R_{t}\left(y_{t}(x)\right)+c_{t}\right) \\
& =\left(R_{t}^{-1}\right)_{\beta}^{\alpha} S^{\beta}\left(R_{t}(x)\right)+O_{0}\left(r^{-1}\right),
\end{aligned}
$$

where we used a Taylor expansion to zeroth order yielding a remainder which is $O_{0}\left(r^{-1}\right) .{ }^{33}$ For later purposes we denote the first term in (D30) by

$$
S_{t}^{\alpha}(x):=\left(R_{t}^{-1}\right)_{\beta}^{\alpha} S^{\beta}\left(R_{t}(x)\right) .
$$

The second term in $\mathrm{D} 28$ is $O_{0}\left(r^{-\epsilon}\right)$. We thus conclude that:

$$
Y_{t}^{\alpha}\left(\psi_{t}(x)\right)=S_{t}^{\alpha}(x)+O_{0}\left(r^{-\epsilon}\right) .
$$

$\overline{33}$ Taylor expansions in $(\mathrm{D} 30, \mathrm{D} 43 \mathrm{n}$ and $\overline{\mathrm{D} 56}$ are realized along the lines described in footnote 31 . 
Integrating with respect to $t$ as in (D24), and using that derivatives with respect to $x$ commute with the $t$ integral we conclude:

$$
\psi_{t}^{\alpha}(x)=x^{\alpha}+s_{t}^{\alpha}(\hat{x})+O_{0}\left(r^{-\epsilon}\right)
$$

where

$$
s_{t}^{\alpha}(\hat{x}):=\int_{0}^{t} S_{t^{\prime}}^{\alpha}(\hat{x}) d t^{\prime} .
$$

We thus recover Eq. (D22). We now show (D23) for $n=1$. Let

$$
D_{\beta t}^{\alpha}(x):=\partial_{\beta} \psi_{t}^{\alpha}(x)
$$

Differentiating (D16) with respect to $x^{\beta}$ we find that $D_{\beta t}^{\alpha}(x)$ satisfies the differential equation: ${ }^{34}$

$$
\frac{d}{d t} D_{\beta t}^{\alpha}(x)=M_{\gamma t}^{\alpha}(x) D_{\beta t}^{\gamma}(x)
$$

with

$$
M_{\gamma t}^{\alpha}(x):=\partial_{\gamma} Y_{t}^{\alpha}\left(\psi_{t}(x)\right) .
$$

For fixed $x$ and $\beta,($ D36 $)$ represents an ordinary differential equation for the three-component vector $v^{\alpha}(t)=D_{\beta t}^{\alpha}(x)$. The solution with initial condition $D_{\beta t=0}^{\alpha}(x)=\delta_{\beta}^{\alpha}$ is then given by:

$$
\begin{aligned}
D_{\beta t}^{\alpha}(x) & =\mathrm{T} \exp \left(\int_{0}^{t} M_{t^{\prime}}(x) d t^{\prime}\right)_{\beta}^{\alpha} \\
& =\delta_{\beta}^{\alpha}+\int_{0}^{t} M_{\beta t^{\prime}}^{\alpha}(x) d t^{\prime}+\int_{0}^{t} \int_{0}^{t^{\prime}} M_{\gamma t^{\prime}}^{\alpha}(x) M_{\beta t^{\prime \prime}}^{\gamma}(x) d t^{\prime \prime} d t^{\prime}+\ldots
\end{aligned}
$$

We now describe the asymptotic form of $M_{\gamma t}^{\alpha}(x)$. There are two contribution to this matrix:

$$
M_{\beta t}^{\alpha}(x)=\partial_{\beta}\left(\stackrel{\circ}{\phi *}_{t *}^{-1} S^{\alpha}\right)\left(\psi_{t}(x)\right)+\partial_{\beta}\left(\stackrel{\circ}{\phi}_{t *}^{-1} \delta^{\alpha}\right)\left(\psi_{t}(x)\right) .
$$

For the first term in (D40) we have:

$$
\begin{aligned}
\partial_{\beta}\left(\dot{\phi}_{t *}^{-1} S^{\alpha}\right)\left(\psi_{t}(x)\right) & =\left(R_{t}^{-1}\right)_{\mu}^{\alpha}\left(R_{t}\right)_{\beta}^{\nu} \partial_{\nu} S^{\mu}\left(R_{t}(x)+R_{t}\left(y_{t}(x)\right)+c_{t}\right) \\
& =\left(R_{t}^{-1}\right)_{\mu}^{\alpha}\left(R_{t}\right)_{\beta}^{\nu} \partial_{\nu} S^{\mu}\left(R_{t}(x)\right)+O_{0}\left(r^{-2}\right), \\
& =\partial_{\beta} S_{t}^{\alpha}(x)+O_{0}\left(r^{-2}\right),
\end{aligned}
$$

where we Taylor expanded to zeroth order obtaining a remainder which is $O_{0}\left(r^{-2}\right)$ and used (D31) to express the first term in compact form. The second term in (D40) is $O_{0}\left(r^{-1-\epsilon}\right)$ and we conclude that:

$$
M_{\beta t}^{\alpha}(x)=\partial_{\beta} S_{t}^{\alpha}(x)+O_{0}\left(r^{-1-\epsilon}\right) .
$$

Using (D44) in (D39) we obtain (only the linear term in $M$ contributes, higher order ones

${ }^{34}$ We assume that, as in the compact manifold case, $\psi_{t}(x)$ is smooth as a function from $\mathbb{R} \times \Sigma$ to $\Sigma$. In particular this implies $\partial_{x^{\alpha}} \partial_{t} \psi_{t}(x)=\partial_{t} \partial_{x^{\alpha}} \psi_{t}(x)$ which is what is being used to obtain (D36). Similar considerations are needed when showing the remaining $n>1$ terms of (D23). 
are already $\left.O_{0}\left(r^{-2}\right)\right)$ :

$$
D_{\beta t}^{\alpha}(x)=\delta_{\beta}^{\alpha}+\partial_{\beta} s_{t}^{\alpha}(x)+O_{0}\left(r^{-1-\epsilon}\right),
$$

which corresponds to $n=1$ of Eq. (D23). We now sketch the argument for the general $n$ case. For $n=1,2, \ldots$ Define the tensors:

$$
\begin{aligned}
D_{\beta_{n} \ldots \beta_{1} t}^{\alpha}(x) & :=\partial_{\beta_{n}} \ldots \partial_{\beta_{1}} \psi_{t}^{\alpha}(x) . \\
M_{\beta_{n} \ldots \beta_{1} t}^{\alpha}(x) & :=\left(\partial_{\beta_{n}} \ldots \partial_{\beta_{1}} Y_{t}^{\alpha}\right)\left(\psi_{t}(x)\right) .
\end{aligned}
$$

In order to avoid notational clutter we are omitting an ' $n$ ' label in the name of each tensor; tensors associated to different values of $n$ are only distinguished by the number of indices they have. The $n=1$ case corresponds to the tensors (D35), (D37).

Differentiating (D16) $n$ times, commuting $t$ and $x$ derivatives (see footnote 34) and using chain rule one obtains a system of differential equations in $t$ satisfied by the tensors $D_{\beta_{n} \ldots \beta_{1} t}^{\alpha}(x)$ :

$$
\frac{d}{d t} D_{\beta_{n} \ldots \beta_{1} t}^{\alpha}(x)=\sum_{m=1}^{n} C_{\beta_{n} \ldots \beta_{1} t}^{\gamma_{m} \ldots \gamma_{1}}(x) M_{\gamma_{m} \ldots \gamma_{1} t}^{\alpha}(x),
$$

where [42],

$$
C_{\beta_{n} \ldots \beta_{1} t}^{\gamma_{m} \ldots \gamma_{1}}(x):=\sum D_{\beta_{i_{I_{m}}} \ldots \beta_{i_{I_{m}}} t}^{\gamma_{m}} t(x) \ldots D_{\beta_{i_{I_{1}}} \ldots \beta_{i_{1}} t}^{\gamma_{1}}(x),
$$

where the sum is over partitions of $\{1, \ldots, n\}$ as described for Eq. (C81). For given $n$, Eq. (D48) involves the differentials of order $m \leq n$. The only occurrence of the $n$-th order differential is in the $m=1$ term, for which (D49) becomes:

$$
C_{\beta_{n} \ldots \beta_{1} t}^{\gamma}(x)=D_{\beta_{n} \ldots \beta_{1} t}^{\gamma}(x) .
$$

All remaining $m>1$ sumands of (D48) involve differentials of order strictly less than $n$. If we collectively denote these terms by:

$$
N_{\beta_{n} \ldots \beta_{1} t}^{\alpha}(x):=\sum_{m=2}^{n} C_{\beta_{n} \ldots \beta_{1} t}^{\gamma_{m} \ldots \gamma_{1}}(x) M_{\gamma_{m} \ldots \gamma_{1} t}^{\alpha}(x),
$$

equation (D48) takes the form:

$$
\frac{d}{d t} D_{\beta_{n} \ldots \beta_{1} t}^{\alpha}(x)=M_{\gamma t}^{\alpha}(x) D_{\beta_{n} \ldots \beta_{1} t}^{\gamma}(x)+N_{\beta_{n} \ldots \beta_{1} t}^{\alpha}(x) .
$$

For fixed $x$, and $\beta_{n} \ldots \beta_{1}$, Eq. (D52) represents an inhomogenous ordinary linear differential equation in $t$ for the three dimensional vector $v^{\alpha}(t):=D_{\beta_{n} \ldots \beta_{1} t}^{\alpha}(x), \alpha=1,2,3$. Since the source term $N_{\beta_{n} \ldots \beta_{1} t}^{\alpha}(x)$ depends on the differentials of order smaller than $n$, the solutions can be obtained iteratively. For $n>1$, the general solution to (D52) with initial condition $D_{\beta_{n} \ldots \beta_{1} t=0}^{\alpha}(x)=0$ is given by:

$$
D_{\beta_{n} \ldots \beta_{1} t}^{\alpha}(x)=D_{\mu t}^{\alpha}(x) \int_{0}^{t}\left(D^{-1}\right)_{\nu t^{\prime}}^{\mu}(x) N_{\beta_{n} \ldots \beta_{1} t^{\prime}}^{\nu}(x) d t^{\prime},
$$

where $\left(D^{-1}\right)_{\beta t}^{\alpha}(x)$ is the inverse matrix of $D_{\beta t}^{\alpha}(x)$ (the invertibilty of this matrix follows from 
the fact that it is the differential of a diffeomorphism). In order to determine the asymptotic form of (D53) we need to study the fall-offs of (D51). This requires knowledge of the fall-offs of the differentials of $\psi_{t}$ up to order $n-1$ (which we assume are given) as well as knowledge of the fall-offs of (D47). For the latter there are two contributions:

$$
M_{\beta_{n} \ldots \beta_{1} t}^{\alpha}(x)=\left(\partial_{\beta_{n}} \ldots \partial_{\beta_{1}}\left(\dot{\phi}_{t *}^{-1} S^{\alpha}\right)\left(\psi_{t}(x)\right)+\left(\partial_{\beta_{n}} \ldots \partial_{\beta_{1}}\left(\dot{\phi}_{t *}^{-1} \delta^{\alpha}\right)\left(\psi_{t}(x)\right) .\right.\right.
$$

For the first term in (D54) we have:

$$
\begin{aligned}
\left(\partial_{\beta_{n}} \ldots \partial_{\beta_{1}} \stackrel{\circ}{\phi}_{t *}^{-1} S^{\alpha}\right)\left(\psi_{t}(x)\right) & =\left(R_{t}^{-1}\right)_{\mu}^{\alpha}\left(R_{t}\right)_{\beta_{n}}^{\nu_{n}} \ldots\left(R_{t}\right)_{\beta_{1}}^{\nu_{1}} \partial_{\nu_{n}} \ldots \partial_{\nu_{1}} S^{\mu}\left(\stackrel{\phi}{\phi}_{t}\left(\psi_{t}(x)\right)\right) \\
& =\partial_{\beta_{n}} \ldots \partial_{\beta_{1}} S_{t}^{\alpha}(x)+O_{0}\left(r^{-n-1}\right)
\end{aligned}
$$

where we Taylor expanded to zeroth order obtaining a remainder which is $O_{0}\left(r^{-n-1}\right)$ and used (D31) to express the first term in compact form. The second term in (D54) is $O_{0}\left(r^{-n-\epsilon}\right)$ and so it follows that:

$$
M_{\beta_{n} \ldots \beta_{1} t}^{\alpha}(x)=\partial_{\beta_{n}} \ldots \partial_{\beta_{1}} S_{t}^{\alpha}(x)+O_{0}\left(r^{-n-\epsilon}\right) .
$$

Going now to (D51), one can verify that the highest order term is given by the $m=n$ sumand, for which (D49) becomes the product of $n$ first order differentials:

$$
C_{\beta_{n} \ldots \beta_{1} t}^{\gamma_{n} \ldots \gamma_{1}}(x):=D_{\beta_{n} t}^{\gamma_{n}}(x) \ldots D_{\beta_{1} t}^{\gamma_{1}}(x) .
$$

Using that $D_{\beta t}^{\gamma}(x)=\delta_{\beta}^{\gamma}+O_{0}\left(r^{-1}\right)$ and $(\mathrm{D} 57)$ we conclude:

$$
N_{\beta_{n} \ldots \beta_{1} t}^{\alpha}(x)=\partial_{\beta_{n}} \ldots \partial_{\beta_{1}} S_{t}^{\alpha}(x)+O_{0}\left(r^{-n-\epsilon}\right) .
$$

Using (D59) in (D53) one obtains the $n$-th condition in Eq. (D23).

\section{c. Step 3: Integrating the flow equation (D9)}

A solution to $(\overline{D 9})$ is given by:

$$
g_{t}=\left(\phi_{t}\right)_{*}\left(\mathrm{~T} e^{\int_{0}^{t}\left(\phi_{t^{\prime}}^{-1}\right)_{*}(\Lambda) d t^{\prime}}\right),
$$

where $\mathrm{T}$ denotes the ordered matrix product so that

$$
\frac{d}{d t} \mathrm{~T}^{\int_{0}^{t}\left(\phi_{t^{\prime}}^{-1}\right)_{*}(\Lambda) d t^{\prime}}=\left(\phi_{t}^{-1}\right)_{*}(\Lambda) \mathrm{T} e^{\int_{0}^{t}\left(\phi_{t^{\prime}}^{-1}\right)_{*}(\Lambda) d t^{\prime}} .
$$

We assume that (D60) is the unique solution to (D9) with initial condition $g_{t=0}=1$. It is then straightforward to verify that $a_{t}=\left(g_{t}, \phi_{t}\right)$ satisfies the one-parameter subgroup property $a_{t} a_{s}=a_{t+s}$ with the product rule given by Eq. (7.7).

Next, we argue that the explicit expression (D60) satisfies the analogue of the fall-offs described in Eq. (7.11). Consider first the $S U(2)$-valued function arising from the time ordered exponential in (D60) satisfying (D61). For $\Lambda$ and $\xi^{a}$ as in (D5), (D6) and the 
smooth version of composition property (C74) described in section C 5 we obtain

$$
\left(\phi_{t}^{-1}\right)_{*} \Lambda=\AA+(\text { even }) r^{-1}+O\left(r^{-1-\epsilon}\right) .
$$

Note that here and in the following equations, the terms (even) $r^{-1}+O\left(r^{-1-\epsilon}\right)$ are in general $t$-dependent. Let

$$
h_{t}:=e^{-t \AA^{\prime}} \mathrm{T} e^{\int_{0}^{t}\left(\phi_{t^{\prime}}^{-1}\right)_{*}(\Lambda) d t^{\prime}} .
$$

It satisfies

$$
\frac{d}{d t} h_{t}=\tilde{\Lambda}_{t} h_{t}
$$

with

$$
\tilde{\Lambda}_{t}=\left(\phi_{t}^{-1}\right)_{*}\left(e^{-t \AA}(\Lambda-\AA) e^{t \AA}\right)=(\text { even }) r^{-1}+O\left(r^{-1-\epsilon}\right),
$$

where again we used the composition property (C74). The expression for $h_{t}$ can then be written as:

$$
h_{t}=\mathrm{T} e^{\int_{0}^{t} \tilde{\Lambda}_{t^{\prime}} d t^{\prime}}
$$

Writing down the explicit power series defining the ordered matrix product and using the fall-off in (D65) one finds

$$
h_{t}=\mathbf{1}+(\text { even }) r^{-1}+O\left(r^{-1-\epsilon}\right)
$$

From Eqns. (D63), (D60), and composition property (C74) we find:

$$
g_{t}=e^{t \AA}+(\text { even }) r^{-1}+O\left(r^{-1-\epsilon}\right)
$$

which is what we wanted to show. ${ }^{35}$

\section{Appendix E: Supplementary material for section VIII}

\section{Finiteness of $\alpha(a, E)$}

From the fall-offs of $g$ and its derivative:

$$
g=\stackrel{\circ}{g}+(\text { even }) r^{-1}+O\left(r^{-1-\epsilon}\right), \quad \partial_{a} g=(\text { odd }) r^{-2}+O\left(r^{-2-\epsilon}\right),
$$

we find:

$$
\begin{aligned}
\partial_{a} g g^{-1} & =\partial_{a} g\left(\stackrel{\circ}{g}^{-1}+(\text { even }) r^{-1}+O\left(r^{-1-\epsilon}\right)\right) \\
& =\partial_{a}\left(g \stackrel{\circ}{g}^{-1}\right)+(\text { odd }) r^{-3}+O\left(r^{-3-\epsilon}\right),
\end{aligned}
$$

where we used that $\partial_{a} \stackrel{\circ}{g}^{-1}=0$. Using $(\mathrm{E} 1),(\mathrm{E} 2)$ and the fact that $a \cdot E^{a}$ satisfies the fall-off conditions (2.1),

$$
a \cdot E^{\alpha}=\stackrel{\circ}{E}^{\alpha}+(\text { even }) r^{-1}+O\left(r^{-1-\epsilon}\right)
$$

${ }^{35}$ Note that $\AA=\Lambda_{R}=\theta$ (see equation (D7) ) so that the rotation of the fiducial flat triad $\stackrel{\circ}{E}^{a}$ by $e^{t \AA}$ is exactly 'undone' by the leading order part of $\phi_{t}$. 
the asymptotic form of the first term in 8.4 is found to be:

$$
\operatorname{Tr}\left[\left(a \cdot E^{\alpha}\right) \partial_{\alpha} g g^{-1}\right]=\partial_{\alpha} \operatorname{Tr}\left[\stackrel{\circ}{E}^{\alpha}\left(g \stackrel{\circ}{ }^{-1}\right)\right]+(\text { odd }) r^{-3}+O\left(r^{-3-\epsilon}\right),
$$

where we used $\partial_{\alpha} \stackrel{\circ}{E}^{\alpha}=0$ to bring $\stackrel{\circ}{E}^{\alpha}$ inside the derivative.

We now focus on the total derivative term in 8.4. Using that $\operatorname{Tr}\left[E^{a}\right]=\operatorname{Tr}\left[\stackrel{\circ}{E}^{a}\right]=0$ together with, (E1), (E3), we find:

$$
\operatorname{Tr}\left[\left(E^{\alpha}-\stackrel{\circ}{E}^{\alpha}\right) g \stackrel{\circ}{g}^{-1}\right]=(\text { even }) r^{-2}+O\left(r^{-2-\epsilon}\right),
$$

from which we obtain the following asymptotic form for the second term in 8.4 :

$$
-\partial_{\alpha} \operatorname{Tr}\left[E^{\alpha} g \stackrel{\circ}{g}^{-1}\right]=-\partial_{\alpha} \operatorname{Tr}\left[\stackrel{\circ}{E}^{\alpha}\left(g \stackrel{\circ}{g}^{-1}\right)\right]+(\operatorname{odd}) r^{-3}+O\left(r^{-3-\epsilon}\right) .
$$

Adding (E4) and (E6) we find

$$
\rho(a, E)=(\text { odd }) r^{-3}+O\left(r^{-3-\epsilon}\right) .
$$

so that $\alpha(a, E)$ is finite.

We conclude with two observations related to Eq. (E5). First, we note that using (E5), $\alpha(a, E)$ can alternatively be written as,

$$
\alpha(a, E)=\int_{\Sigma} \operatorname{Tr}\left[a \cdot E^{a} \partial_{a} g g^{-1}\right]-\oint_{\infty} d S_{a} \operatorname{Tr}\left[\stackrel{\circ}{E}^{a} g \stackrel{\circ}{g}^{-1}\right] .
$$

The form (E8) makes it explicit that the 'additional' surface term is sensitive only to the limiting value of $E^{a}$. Second, we note that for $\bar{E}^{a} \in \mathcal{E}$ we have

$$
\operatorname{Tr}\left[\bar{E}^{\alpha} g \stackrel{\circ}{g}^{-1}\right]=(\text { even }) r^{-2}+O\left(r^{-2-\epsilon}\right)
$$

so that

$$
\oint_{\infty} d S_{a} \operatorname{Tr}\left[\bar{E}^{a} g \stackrel{\circ}{g}^{-1}\right]=0
$$

Eq. (E9) follows from the same argument given for Eq. (E5). Eq. E10 implies that if one uses formula 8.3 with $\bar{E}^{a} \in \mathcal{E}$, one recovers the phase factor for 'barred' electric fields, Eq. 7.31. This in turn implies Eq. 8.12.

\section{Eq. (8.8)}

Let $a=(g, \phi), a^{\prime}=\left(g^{\prime}, \phi^{\prime}\right)$ and

$$
a^{\prime \prime}=\left(g^{\prime \prime}, \phi^{\prime \prime}\right):=a a^{\prime}=\left(g \phi_{*} g^{\prime}, \phi \phi^{\prime}\right) .
$$

The phase associated to $a^{\prime \prime}$ is

$$
\alpha\left(a^{\prime \prime}, E\right)=\int_{\Sigma} \rho\left(a^{\prime \prime}, E\right)
$$


with $\rho$ in 8.4 evaluated on the group element (E11):

$$
\begin{aligned}
\rho\left(a^{\prime \prime}, E\right) & =\operatorname{Tr}\left[a \cdot\left(a^{\prime} \cdot E^{a}\right) \partial_{a}\left(g \phi_{*} g^{\prime}\right) \phi_{*} g^{\prime-1} g^{-1}\right]-\partial_{a} \operatorname{Tr}\left[E^{a} g^{\prime \prime} \stackrel{\circ}{g}^{\prime \prime-1}\right] \\
& =\operatorname{Tr}\left[a \cdot\left(a^{\prime} \cdot E^{a}\right) \partial_{a} g g^{-1}\right]+\phi_{*} \operatorname{Tr}\left[a^{\prime} \cdot E^{a} \partial_{a} g^{\prime} g^{\prime-1}\right]-\partial_{a} \operatorname{Tr}\left[E^{a} g^{\prime \prime} \stackrel{\circ}{g}^{\prime \prime-1}\right] .
\end{aligned}
$$

We now focus on the total derivative term in (E14). Using the expansion (E1) for $g$ and $g^{\prime}$ and the fact that $\phi_{*} \stackrel{\circ}{g}^{\prime}=\stackrel{\circ}{g}^{\prime}$ (since $\stackrel{\circ}{g}^{\prime}$ is a constant) we find:

$$
\begin{aligned}
\stackrel{\circ}{g}^{\prime \prime} & =\stackrel{\circ}{g} \stackrel{\circ}{ }^{\prime} \\
g^{\prime \prime} & =g \stackrel{\circ}{g}^{\prime}+\stackrel{\circ}{g} \phi_{*} g^{\prime}-\stackrel{\circ}{g} \stackrel{g}{ }^{\prime}+(\text { even }) r^{-2}+O\left(r^{-2-\epsilon}\right) \\
g^{\prime \prime} \stackrel{\circ}{g}^{\prime \prime-1} & =g \stackrel{\circ}{g}^{-1}+\stackrel{\circ}{g}\left(\phi_{*} g^{\prime} \stackrel{\circ}{g}^{-1}\right) \stackrel{\circ}{g}^{-1}-\mathbf{1}+(\text { even }) r^{-2}+O\left(r^{-2-\epsilon}\right) .
\end{aligned}
$$

The relevant terms appearing in $\operatorname{Tr}\left[E^{a} g^{\prime \prime} \stackrel{\circ}{g}^{\prime \prime-1}\right]$ are then:

$$
\operatorname{Tr}\left[E^{\alpha} g \stackrel{\circ}{g}^{-1}\right]=\operatorname{Tr}\left[a^{\prime} \cdot E^{\alpha} g \stackrel{\circ}{g}^{-1}\right]+(\text { even }) r^{-2}+O\left(r^{-2-\epsilon}\right),
$$

where the equality follows from Eq. (E9) together with the fact that $a^{\prime} \cdot E^{a}-E^{a} \in \mathcal{E}$, and

$$
\begin{aligned}
\operatorname{Tr}\left[\stackrel{\circ}{g}^{-1} E^{\alpha} \stackrel{\circ}{g} \phi_{*} g^{\prime} \stackrel{\circ}{g}^{\prime-1}\right] & =\phi_{*} \operatorname{Tr}\left[\phi_{*}^{-1}\left(\stackrel{\circ}{g}-1 E^{\alpha} \stackrel{\circ}{g}\right) g^{\prime} \stackrel{\circ}{g}^{\prime-1}\right] \\
& =\phi_{*} \operatorname{Tr}\left[E^{\alpha} g^{\prime} \stackrel{\circ}{g}^{\prime-1}\right]+(\text { even }) r^{-2}+O\left(r^{-2-\epsilon}\right),
\end{aligned}
$$

where in the last equality we again used Eq. (E9) since,

$$
\phi_{*}^{-1}\left(\stackrel{\circ}{g}-1 E^{\alpha} \stackrel{\circ}{g}\right)=\stackrel{\circ}{E}^{\alpha}+(\text { even }) r^{-1}+O\left(r^{-1-\epsilon}\right) .
$$

From (E17), E18) and (E19) it follows that

$$
\operatorname{Tr}\left[E^{a} g^{\prime \prime} \stackrel{\circ}{g}^{\prime \prime-1}\right]=\operatorname{Tr}\left[a^{\prime} \cdot E^{\alpha} g \stackrel{\circ}{g}^{-1}\right]+\phi_{*} \operatorname{Tr}\left[E^{\alpha} g^{\prime} \stackrel{\circ}{ }^{\prime-1}\right]+X^{a},
$$

where,

$$
X^{a}=(\text { even }) r^{-2}+O\left(r^{-2-\epsilon}\right),
$$

collects the remainder terms arising in (E17), (E18) and (E19).

Using Eq. (E21) in (E14), and recalling the definition of $\rho$ (Eq. (8.4), Eq. (E14) becomes:

$$
\rho\left(a^{\prime \prime}, E\right)=\rho\left(a^{\prime} \cdot E, a\right)+\phi_{*} \rho\left(a^{\prime}, E\right)+\partial_{a} X^{a},
$$

Integrating $($ E23 $)$ over $\Sigma$ we find that the total derivative term in $($ E23) give zero contribution since $\oint_{\infty} d S_{a} X^{a}=0$ for $X^{a}$ as in $(\mathrm{E} 22)$. Finally, using $\int_{\Sigma} \phi_{*} \rho\left(a^{\prime}, E\right)=\int_{\Sigma} \rho\left(a^{\prime}, E\right)=\alpha\left(a^{\prime}, E\right)$ we obtain (8.8).

\section{Properties of the rank sets}

In this appendix we show that the rank sets 8.18), 8.19), 8.20) as well as the intersections given in Eq. (8.23) can be decomposed into finite union of semianalytic submanifolds. In [9] this result followed from the fact that the sets can be described in terms of semianalytic functions, together with compactness of $\Sigma$. The rank sets here are also described in terms of semianalytic functions (see Eqns. (6.4)-(6.9) of [9]). Below we adapt the proof to the present case by dividing $\Sigma$ into an 'inside' compact region and an 'outside' non-compact 
region where $E^{a}$ is of rank 3 .

In the following we work with a given fixed electric field $E^{a}$ of the type described in section II. The fall off conditions (2.1) allow us to find $r_{0}$ such that $E^{a}$ is of rank 3 for points outside the 2-sphere $S_{r_{o}}$ (with respect to the Cartesian chart $\left\{x^{\alpha}\right\}$ ). Let us fix $r>r_{0}$, and denote by $\Sigma_{r}$ the compact, 'inside' region so that in the Cartesian chart $\Sigma \backslash \Sigma_{r}=\left\{\vec{x} \in \mathbb{R}^{3}\right.$ : $\left.\left(x^{1}\right)^{2}+\left(x^{2}\right)^{2}+\left(x^{3}\right)^{2}>r^{2}\right\}$. We can describe $\Sigma_{r}$ in terms of a semianalytic function $f_{r}$ such that $\Sigma_{r}=\left\{f_{r}(x)=0\right\}$ and $\Sigma \backslash \Sigma_{r}=\left\{f_{r}(x)>0\right\}$. We define $f_{r}$ by

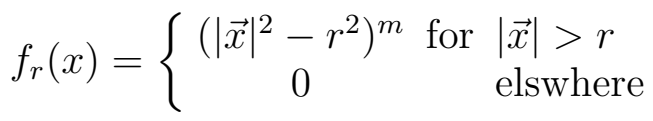

where the first line refers to the Cartesian chart $\left\{x^{\alpha}\right\}$ with $|\vec{x}| \equiv \sqrt{\left(x^{1}\right)^{2}+\left(x^{2}\right)^{2}+\left(x^{3}\right)^{2}}$, and $m>k$.

It follows that $V_{2}^{E}=\left\{f_{r}>0\right\} \cup\left(\left\{f_{r}=0\right\} \cap V_{2}^{E}\right)$ and $V_{n}^{E}=\left\{f_{r}=0\right\} \cap V_{n}^{E}$ for $n=0,1$. Thus, all rank sets and their intersections (8.23) are of the form $X=\left\{f_{r}=0\right\} \cap_{i=1}^{n}\left\{f_{i} s_{i} 0\right\}$ for some given semianalytic functions $f_{i}: \Sigma \rightarrow \mathbb{R}, i=1, \ldots n$ and choices of symbols $s_{i} \in\{=,<,>\}$. We now adapt the proof given in appendix B of [9] to show that such $X$ is a finite unions of submanifolds. In the following the index $\alpha$ stands for $\alpha=r, 1, \ldots, n$ so that $X=\cap_{\alpha}\left\{f_{\alpha} s_{\alpha} 0\right\}$ with $s_{r}="="$.

Let $\left\{\chi_{I}, U_{I}\right\}$ be a semianalytic atlas of $\Sigma$ compatible with the functions $\left\{f_{\alpha}\right\}$. That is, each $U_{I}$ admits a semianalytic partition compatible with the functions $f_{\alpha}$. We recall that a semianalytic partition means a decomposition of the form:

$$
U_{I}^{\prime}:=\chi_{I}\left(U_{I}\right)=\cup_{\sigma^{I}} V_{\sigma^{I}}^{I},
$$

where $\sigma^{I}:=\sigma_{1}^{I}, \ldots, \sigma_{n_{I}}^{I}$ is a sequence of $n_{I}$ symbols $<,>$ or $=$ and $V_{\sigma^{I}}^{I}$ is defined in terms of analytic functions $h_{i}^{I}, i=1, \ldots n_{I}$, on $U_{I}^{\prime}$ by $V_{\sigma^{I}}^{I}:=\cap_{i=1}^{n_{I}}\left\{h_{i}^{I} \sigma_{i}^{I} 0\right\}$. The compatibility of the functions $f_{\alpha}$ with the partition means there exists analytic functions $\left\{f_{\alpha \sigma^{I}}^{I}: U_{I}^{\prime} \rightarrow \mathbb{R}\right\}$ such that

$$
\left.f_{\alpha} \circ \chi_{I}^{-1}\right|_{V_{\sigma}^{I}}=\left.f_{\alpha \sigma}^{I}\right|_{V_{\sigma}^{I}} .
$$

Thus, on each local chart the set of interest takes the form:

$$
\chi_{I}\left(X \cap U_{I}\right)=\cup_{\sigma}\left(\left\{\cap_{\alpha} f_{\alpha \sigma}^{I} s_{\alpha} 0\right\} \cap\left\{h_{1}^{I}(x) \sigma_{1} 0\right\} \ldots \cap\left\{h_{n_{I}}^{I}(x) \sigma_{n_{I}} 0\right\}\right) .
$$

The sets featuring in the union $(\mathrm{E} 27)$ can be realized as sets of a new partition of $U_{I}^{\prime}$ defined in terms of the functions $\left\{\left\{h_{i}^{I}\right\},\left\{f_{\alpha \sigma^{I}}^{I}\right\}\right.$. By proposition A.9 of [22] it follows that every $x \in U_{I}$ has an open neighborhood $U_{I}^{x}$ such that

$$
\chi_{I}\left(X \cap U_{I}^{x}\right)=\text { finite union of analaytic submanifolds. }
$$

This in turn implies that $X \cap U_{I}^{x}$ is a finite union of semianalytic submanifolds of $\Sigma$. Now, the (uncountable) collection of such open sets $\left\{U_{I}^{x}\right\}$ cover $\Sigma$ and in particular $\Sigma_{r}$. Since the latter is compact, it follows there exists a finite subfamily $\left\{W_{\beta}\right\} \subset\left\{U_{I}^{x}\right\}$ that covers $\Sigma_{r}$. Thus $\Sigma_{r} \subset \cup_{\beta} W_{\beta}$. Since $X \subset \Sigma_{r}$ it follows that

$$
X=\cup_{\alpha}\left(X \cap W_{\alpha}\right)=\text { finite union of semianalytic submanifolds. }
$$


[1] A. Ashtekar, J. Lewandowski, D. Marolf, J. Mourao and T. Thiemann, "Quantization of diffeomorphism invariant theories of connections with local degrees of freedom," J. Math. Phys. 36 (1995) 6456 [gr-qc/9504018]

[2] C. Rovelli and L. Smolin, "Spin networks and quantum gravity," Phys. Rev. D 52, 5743 (1995) [gr-qc/9505006]

[3] J. C. Baez, "Spin network states in gauge theory," Adv. Math. 117, 253 (1996) [gr-qc/9411007]

[4] A. Ashtekar and J. Lewandowski, "Quantum theory of geometry. 1: Area operators," Class. Quant. Grav. 14, A55 (1997) [gr-qc/9602046]

[5] A. Ashtekar, "New perspectives in canonical gravity" , Bibliopolis (1988)

[6] T. A. Koslowski, "Dynamical Quantum Geometry (DQG Programme)," arXiv:0709.3465 [gr$\mathrm{qc}]$.

[7] H. Sahlmann, "On loop quantum gravity kinematics with non-degenerate spatial background," Class. Quant. Grav. 27, 225007 (2010) [arXiv:1006.0388 [gr-qc]]

[8] T. Koslowski and H. Sahlmann, "Loop quantum gravity vacuum with nondegenerate geometry," SIGMA 8, 026 (2012) [arXiv:1109.4688 [gr-qc]]

[9] M. Campiglia and M. Varadarajan, "The Koslowski-Sahlmann representation: gauge and diffeomorphism invariance," Class. Quant. Grav. 31, 075002 (2014) [arXiv:1311.6117 [gr-qc]]

[10] M. Arnsdorf and S. Gupta, "Loop quantum gravity on noncompact spaces," Nucl. Phys. B $\mathbf{5 7 7}, 529$ (2000) [gr-qc/9909053].

[11] T. Thiemann and O. Winkler, "Gauge field theory coherent states (GCS) 4: Infinite tensor product and thermodynamical limit," Class. Quant. Grav. 18, 4997 (2001) [hep-th/0005235]

[12] M. Varadarajan, "The generator of spatial diffeomorphisms in the Koslowski-Sahlmann representation," Class. Quant. Grav. 30 (2013) 175017 [arXiv:1306.6126 [gr-qc]]

[13] M. Campiglia and M. Varadarajan "The Koslowski-Sahlmann representation: Quantum Configuration Space" arXiv:1406.0579 [gr-qc]

[14] "Spin 1/2 From Gravity," J. L. Friedman and R. D. Sorkin, Phys. Rev. Lett. 44, 1100 (1980).

[15] M. Arnsdorf and R. S. Garcia, "Existence of spinorial states in pure loop quantum gravity," Class. Quant. Grav. 16, 3405 (1999) [gr-qc/9812006]

[16] T. Regge and C. Teitelboim, "Role of Surface Integrals in the Hamiltonian Formulation of General Relativity," Annals Phys. 88, 286 (1974).

[17] R. Beig and N. ó Murchadha, "The Poincare group as the symmetry group of canonical general relativity," Annals Phys. 174, 463 (1987)

[18] T. Thiemann, "Generalized boundary conditions for general relativity for the asymptotically flat case in terms of Ashtekar's variables," Class. Quant. Grav. 12, 181 (1995)

[19] M. Campiglia, "Note on the phase space of asymptotically flat gravity in Ashtekar-Barbero variables"

[20] T. Thiemann, "Modern canonical quantum general relativity", Cambridge, UK: Cambridge Univ. Pr. (2007)

[21] A. Ashtekar, A. Corichi and J. A. Zapata, "Quantum theory of geometry III: Noncommutativity of Riemannian structures," Class. Quant. Grav. 15, 2955 (1998) [gr-qc/9806041]

[22] J. Lewandowski, A. Okołów, H. Sahlmann, T. Thiemann, "Uniqueness of the diffeomorphism invariant state on the quantum holonomy-flux algebras", Comm. Math. Phys. 267 703-733 
(2006)

[23] A. D. Rendall, "Comment on a paper of Ashtekar and Isham," Class. Quant. Grav. 10, 605 (1993)

[24] A. Ashtekar and J. Lewandowski, "Representation theory of analytic holonomy C* algebras," gr-qc/9311010.

[25] J. Glimm and A. M. Jaffe, "Quantum Physics. A Functional Integral Point Of View," New York, USA: Springer (1987)

[26] D. Marolf and J. M. Mourao, "On the support of the Ashtekar-Lewandowski measure," Commun. Math. Phys. 170, 583 (1995) [hep-th/9403112]

[27] J. M. Velhinho, "On the structure of the space of generalized connections," Int. J. Geom. Meth. Mod. Phys. 1, 311 (2004) [math-ph/0402060]

[28] A. Ashtekar and J. Lewandowski, "Projective techniques and functional integration for gauge theories," J. Math. Phys. 36, 2170 (1995) [gr-qc/9411046]

[29] A. Ashtekar and J. Lewandowski, "Background independent quantum gravity: A Status report," Class. Quant. Grav. 21, R53 (2004)

[30] S. G. Krantz and H. R. Parks, "The Implicit Function Theorem: History, Theory, and Applications", Birkhäuser (2012)

[31] S. Lang, "Introduction to Differentiable Manifolds", Springer (2002)

[32] L. Smolin, "Positive energy in quantum gravity," arXiv:1406.2611 [gr-qc]

[33] T. Thiemann, "Quantum spin dynamics (QSD)," Class. Quant. Grav. 15, 839 (1998) [grqc/9606089]

[34] A. Laddha, "Hamiltonian constraint in Euclidean LQG revisited: First hints of off-shell Closure," arXiv:1401.0931 [gr-qc]

[35] A. Henderson, A. Laddha and C. Tomlin, "Constraint algebra in loop quantum gravity reloaded. I. Toy model of a $U(1)^{3}$ gauge theory," Phys. Rev. D 88, no. 4, 044028 (2013) [arXiv:1204.0211 [gr-qc]]

[36] A. Henderson, A. Laddha and C. Tomlin, "Constraint algebra in loop quantum gravity reloaded. II. Toy model of an Abelian gauge theory: Spatial diffeomorphisms," Phys. Rev. D 88, no. 4, 044029 (2013) [arXiv:1210.3960 [gr-qc]]

[37] C. Tomlin and M. Varadarajan, "Towards an Anomaly-Free Quantum Dynamics for a Weak Coupling Limit of Euclidean Gravity," Phys. Rev. D 87, no. 4, 044039 (2013) [arXiv:1210.6869 [gr-qc]]

[38] M. Varadarajan, "Towards an Anomaly-Free Quantum Dynamics for a Weak Coupling Limit of Euclidean Gravity: Diffeomorphism Covariance," Phys. Rev. D 87 (2013) 4, 044040 [arXiv:1210.6877 [gr-qc]]

[39] S. Sengupta, "Quantum geometry with a nondegenerate vacuum: a toy model," Phys. Rev. D 88, 064016 (2013) [arXiv:1306.6013 [gr-qc]]

[40] S. Sengupta, "Asymptotic Flatness and Quantum Geometry," Class. Quant. Grav. 31, 085005 (2014) [arXiv:1309.5266 [gr-qc]

[41] M. Varadarajan, "Towards new background independent representations for loop quantum gravity," Class. Quant. Grav. 25, 105011 (2008) [arXiv:0709.1680 [gr-qc]]

[42] M. Grosser, M. Kunzinger, M. Oberguggenberger, R. Steinbauer, "Geometric Theory of Generalized Functions with Applications to General Relativity", Springer (2001)

[43] A. Ashtekar, "Logarithmic ambiguities in the description of spatial infinity", Foundations of physics 15 no. 4, 419-431 (1985) 